\title{
Carcinogenesis and Reactive Oxygen Species Signaling: Interaction of the NADPH Oxidase NOX1-5 and Superoxide Dismutase 1-3 Signal Transduction Pathways
}

\author{
Alessia Parascandolo and Mikko O. Laukkanen*
}

\begin{abstract}
Significance: Reduction/oxidation (redox) balance could be defined as an even distribution of reduction and oxidation complementary processes and their reaction end products. There is a consensus that aberrant levels of reactive oxygen species (ROS), commonly observed in cancer, stimulate primary cell immortalization and progression of carcinogenesis. However, the mechanism how different ROS regulate redox balance is not completely understood. Recent Advances: In the current review, we have summarized the main signaling cascades inducing NADPH oxidase NOX1-5 and superoxide dismutase (SOD) 1-3 expression and their connection to cell proliferation, immortalization, transformation, and $\mathrm{CD} 34^{+}$cell differentiation in thyroid, colon, lung, breast, and hematological cancers.

Critical Issues: Interestingly, many of the signaling pathways activating redox enzymes or mediating the effect of ROS are common, such as pathways initiated from $\mathrm{G}$ protein-coupled receptors and tyrosine kinase receptors involving protein kinase A, phospholipase $\mathrm{C}$, calcium, and small GTPase signaling molecules.

Future Directions: The clarification of interaction of signal transduction pathways could explain how cells regulate redox balance and may even provide means to inhibit the accumulation of harmful levels of ROS in human pathologies. Antioxid. Redox Signal. 30, 443-486.
\end{abstract}

Keywords: redox signaling, NADPH oxidase NOX, superoxide dismutase, tyrosine kinase receptor, G proteincoupled receptor

\section{Table of Contents}

I. Introduction

A. Superoxide anion and hydrogen peroxide 444

B. Concentration-dependent effect of $\mathrm{H}_{2} \mathrm{O}_{2}$ and $\mathrm{O}_{2}{ }^{-{ }^{-}}$on signal transduction 445

II. NADPH Oxidase NOX1-5 Family 446

III. NADPH Oxidase NOX1 446

A. NADPH oxidase NOX1 in tumorigenesis 446

IV. NADPH Oxidase NOX2 448

A. NADPH oxidase NOX2 complex formation $\quad 448$

V. NADPH Oxidase NOX3 4449

VI. NADPH Oxidase NOX4 449

A. NADPH oxidase NOX4 in tumorigenesis 449

VII. NADPH Oxidase NOX5 450

A. NADPH oxidase NOX5 in tumorigenesis $\quad 451$

VIII. SOD1-3 Family 451

Reviewing Editors: Rogerio Castilho, Mario Chiariello, Rinesh Godfrey, Agnes Görlach, Domenico Grieco, Herve Kovacic, Elaine Leung, Giovanni Li Volti, Ruy Louzada, and Jennifer Meitzler

IRCCS SDN, Naples, Italy.

*Current affiliation: Institute of Experimental Endocrinology and Oncology (CNR), Naples, Italy.

(C) Alessia Parascandolo and Mikko O. Laukkanen, 2019; Published by Mary Ann Liebert, Inc. This Open Access article is distributed under the terms of the Creative Commons License (http://creativecommons.org/licenses/by/4,0), which permits unrestricuted use, distribution, and reproduction in any medium, provided the original work is properly cited. 
IX. Copper Zinc SOD, CuZnSOD, SOD1 451

A. SOD1 in tumorigenesis 452

X. Manganese SOD, MnSOD, SOD2 453

A. SOD2 in tumorigenesis

XI. Extracellular SOD, EC-SOD, SOD3 454

A. SOD3 in tumorigenesis

XII. Interaction of NOX1-5- and SOD1-3-Associated Signaling 456

A. GPCR signaling 456

B. $\mathrm{Ca}^{2+}$ signaling 458

C. RTK and small GTPase signaling 458

D. Oncogene signaling 459

XIII. Altered ROS Levels Are Associated with Progression of Tumorigenesis 459

XIV. ROS in Thyroid Cancer 460

A. Redox-related signaling in thyroid tumorigenesis 460

B. NOX1-5 and SOD1-3 expression in thyroid cancer 461

C. Percentage change in redox gene expression in PTC 463

XV. ROS in Colon Cancer 464

A. Progression of colon cancer 464

B. WNT signaling in the normal colon and in colon cancer development 465

C. NOX1-5 and SOD1-3 gene expression in colon tumorigenesis 465

XVI. ROS in Breast Cancer 466

A. ROS-related characteristics of breast cancer 466

B. NOX1-5 and SOD1-3 gene expression in breast tumorigenesis 467

XVII. ROS in Lung Cancer 467

A. ROS-related characteristics of lung cancer 467

B. NOX1-5 and SOD1-3 gene expression in lung tumorigenesis 469

XVIII. ROS in Hematological Cancers 469

A. ROS in CD34 HSC differentiation 469

B. ROS in hematological cancers and therapy 470

XIX. Summary and Conclusions 471

\section{Introduction}

\section{A. Superoxide anion and hydrogen peroxide}

$\mathbf{R}$ EACTIVE OXYGEN SPECIES (ROS), a heterogeneous group of reactive oxygen derivatives, are involved in cellular signal transduction events regulating growth, differentiation, survival, and apoptosis. The effect of ROS on oxidative cell signaling depends on the type of ROS produced, concentration of ROS, localization of ROS, and persistence of ROS production. Increased or decreased production of ROS has a drastic impact on cell fate, thus reflecting the importance of ROS balance for cellular signal transduction.

Superoxide anion $\left(\mathrm{O}_{2}{ }^{\bullet-}\right)$, produced by NADPH oxidases, and hydrogen peroxide $\left(\mathrm{H}_{2} \mathrm{O}_{2}\right)$, produced by superoxide dismutases (SODs) and by NADPH oxidases, represent intensively investigated ROS. Both ROS function as second messengers in cellular signaling, being able to activate or inactivate signaling pathways, thus regulating the phosphorylation of tyrosine kinase receptors (RTKs) and downstream signaling molecules. ROS affect virtually all normal and pathological conditions, including the function of the normal and injury-related cardiovascular systems $(307,391)$, hematopoiesis $(44,208)$, cancer (90), fibrotic diseases (40, $382)$, aging $(90,98)$, neurodegeneration $(8)$, cellular senescence (98), apoptosis, and cell death $(254,299)$.

The location of NADPH oxidases and SOD enzymes in different cellular membranes and organelles $(31,163,314)$ may influence the physiological roles of these molecules in cells and the signaling pathways regulating cellular functions (Fig. 1A).
$\mathrm{O}_{2}{ }^{\bullet-}$ is a short-lived, highly reactive radical that, in aberrant levels, causes a high number of modifications in cellular functions. Although the NADPH oxidase family of NOX enzymes is an intensively studied source of $\mathrm{O}_{2}{ }^{\bullet-}$ ROS, ROS are also produced from other cellular organelles, such as those of the mitochondrial respiratory chain, composed of complexes I-IV. In mitochondria, the $\mathrm{O}_{2}{ }^{\bullet-}$ radical is produced by complex I, the largest unit in the mitochondrial respiratory chain, which oxidizes NADH to NAD to produce ubiquinone and simultaneously release protons that contribute to ATP production $(325,381)$.

During electron transport, complex III produces four protons that are released into the intermembrane space, creating a transmembrane proton gradient that is later used by ATP synthase to synthesize ATP, and reduces cytochrome C levels, releasing electrons to complex IV. In addition, there is a premature leakage of a small portion of electrons from complex III that, in certain cases, may react with oxygen, resulting in $\mathrm{O}_{2}{ }^{\bullet-}$ formation $(6,68,160)$.

The catalysis of $\mathrm{O}_{2}{ }^{\bullet-}$ to $\mathrm{H}_{2} \mathrm{O}_{2}$ can be spontaneous or catalyzed by SOD enzymes in two half reactions (Fig. 1B). The half-life of $\mathrm{H}_{2} \mathrm{O}_{2}$ is markedly longer than that of $\mathrm{O}_{2}{ }^{\circ-}$. Although in the tissue environment the half-life of $\mathrm{H}_{2} \mathrm{O}_{2}$ is only 1-3 ms (249), in the cell culture environment it may take even 40 min for a single $20-\mu M$ dose of $\mathrm{H}_{2} \mathrm{O}_{2}$ to be completely erased (366). When studying the impact of $\mathrm{H}_{2} \mathrm{O}_{2}$ on cellular homeostasis in vitro, it is important to note that the extracellular $\mathrm{H}_{2} \mathrm{O}_{2}$ concentration is $\sim 10$-fold higher than the intracellular concentration (336), indicating a regulatory mechanism that controls $\mathrm{H}_{2} \mathrm{O}_{2}$ transport into the cells. In 
A

FIG. 1. Redox enzyme NADPH oxidase 1-5 and SOD1-3 expression is influenced by various factors in different cellular localizations. (A) Primary expression sites at cell membranes and cellular organelles. (B) $\mathrm{O}_{2}{ }^{\bullet-}$ is dismutated to $\mathrm{H}_{2} \mathrm{O}_{2}$ in two halfreactions. (C) Stimulation of NOX1 expression. RTK activation induces RASERK1/2 and RAS-p38MAPK signaling pathways, thereby stimulating NOX1 mRNA synthesis. (D) Mitogen stimulation of the PKC pathway induces NOXO1 phosphorylation at Thr154 and Thr341 causing dimer formation with NOXA1 and consequent $\mathrm{O}_{2}{ }^{--}$formation, which is attenuated by MAPK, PKC, and PKA-induced phosphorylation of NOXA1 at Ser172 and Ser282. $\mathrm{H}_{2} \mathrm{O}_{2}$, hydrogen peroxide; mRNA, messenger RNA; NOXA1, NADPH oxidase activator 1 subunit; NOXO1, NADPH oxidase organizer 1 subunit; $\mathrm{O}_{2}{ }^{\bullet-}$, superoxide anion; PKA/AKT, protein kinase A; PKC, protein kinase C; redox, reduction/oxidation; RTK, tyrosine kinase receptor; SOD, superoxide dismutase.

\begin{tabular}{l|l} 
Enzyme & Main expression site \\
\hline NADPH oxidase NOX1 & Caveolae on the plasma membrane \\
\hline NADPH oxidase NOX2 & Phagosomes and on the leading edge of lamellipodia \\
\hline NADPH oxidase NOX3 & Plasma membrane \\
\hline NADPH oxidase NOX4 & Focal adhesions, nucleus, endoplasmic reticulum \\
\hline NADPH oxidase NOX5 & Internal membranes, plasma membrane \\
\hline SOD1 & Cytoplasm, mitochondrial intermembrane space \\
\hline SOD2 & Mitochondria \\
\hline SOD3 & Plasma membrane
\end{tabular}
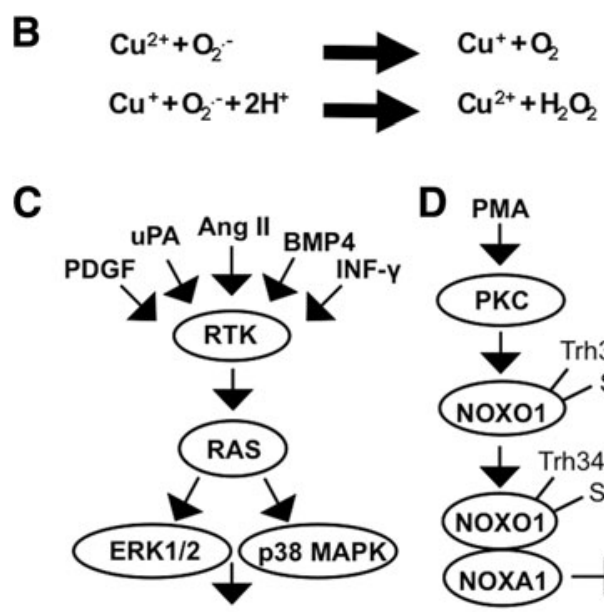

NOX1 mRNA synthesis

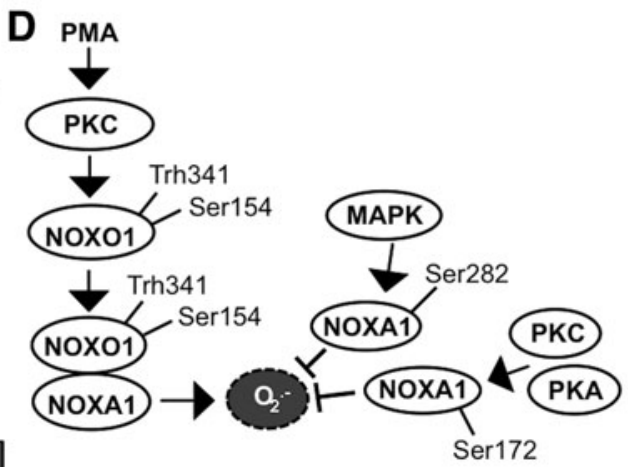

general, in all aerobic organisms, the physiological intracellular $\mathrm{H}_{2} \mathrm{O}_{2}$ concentration varies from 0.001 to $0.5 \mu M$ up to a maximum of $0.7 \mu M$ (340). Although $\mathrm{H}_{2} \mathrm{O}_{2}$ may passively diffuse through membrane structures, a recent article demonstrated that $\mathrm{H}_{2} \mathrm{O}_{2}$ passes the cell membrane, mainly through the aquaporin 3 (AQP3) integral membrane protein channel (239). The observation suggests that AQP3 functions as a regulator of the $\mathrm{H}_{2} \mathrm{O}_{2}$ concentration close to cell membrane-associated signaling molecules, such as RTKs and cell membrane-associated nonreceptor kinases.

\section{B. Concentration-dependent effect of $\mathrm{H}_{2} \mathrm{O}_{2}$ and $\mathrm{O}_{2}{ }^{--}$on signal transduction}

In mammalian cells, such as thyrocytes and hepatocytes, and even in some microbes, $\mathrm{H}_{2} \mathrm{O}_{2}$ has been shown to have growth and survival supportive characteristics at low physiological intracellular levels $(0.001-0.7 \mu M)$, whereas higher levels (20-200 $\mu M)$ induce growth arrest, and levels above that eventually cause cell death $(118,336,339)$, thus suggesting a dose-dependent response in signal transduction, cell growth, and survival. At high concentrations, $\mathrm{H}_{2} \mathrm{O}_{2}$, similar to other ROS, reacts with various cellular macromolecules, causing oxidative stress, DNA damage, mutagenesis, and apoptosis; however, at lower physiological concentrations, $\mathrm{H}_{2} \mathrm{O}_{2}$ modifies cellular signal transduction by stimulating or inhibiting signaling molecule activation $(339,340)$.

The mechanism underlying how ROS activate cell surface RTKs and downstream signal transduction is based on the coordinated action of RTKs and protein tyrosine phospha- tases (PTPs). RTK activation is controlled by PTPs, which inactivate phosphorylated tyrosine amino acids in RTKs simply by removing the phosphate groups. $\mathrm{H}_{2} \mathrm{O}_{2}$-derived activation of RTKs is based on its ability to induce the oxidative inactivation of PTPs by modifying the catalytic site cysteine amino acids. Cysteine residues are reversibly oxidized to sulfenic acid (S-OH), followed by the removal of oxygen in a reaction resulting in cyclic sulfenamide formation. Sulfenamide formation causes a structural change in the catalytic site, exposing oxidatively inactivated cysteine groups to the solvent position. In the newly formed position, the cysteine residues are available as reducing agents, returning PTPs back to the active form $(267,354)$.

It is noteworthy that the response of $\mathrm{H}_{2} \mathrm{O}_{2}$ to cellular functions is not only location and concentration dependent but can also be based on the duration of oxidative stress $(231,250$, 372). Treatment of primary fibroblasts by adding $10 \mu M \mathrm{H}_{2} \mathrm{O}_{2}$ to the cell culture medium every 3 days for 2 weeks to model low-level persistent cell stress reduced the DNA damage repair capacity, causing the accumulation of DNA damage and resulting in telomere shortening, eventually inducing irreversible growth arrest and senescence (82). Therefore, a low level of oxidative stress, which initially may induce cell survival mechanisms, in the long-term may cause irreversible changes in signal transduction, eventually destroying the cell or, alternatively, initiating the cell transformation process.

In general, the imbalanced production of ROS, especially $\mathrm{H}_{2} \mathrm{O}_{2}$, may affect signal transduction by promoting cancer cell survival, proliferation, migration, and drug resistance (21). Although cancer cells become adapted to increased 
ROS levels, the aberrant supraphysiological levels of either $\mathrm{O}_{2}{ }^{\bullet-}$ or $\mathrm{H}_{2} \mathrm{O}_{2}$ markedly influence cell survival by reducing cell cycling and cell proliferation. Therefore, increased ROS levels are frequently used as cytotoxins in cancer patients $(13,23)$.

\section{NADPH Oxidase NOX1-5 Family}

Characteristically, NADPH oxidase isoforms are heteromeric multiunit structures that are activated by the sequential assembly of subunits at cell membranes $(31,213)$. The most extensively studied isoform contains the phagocytic NADPH oxidase gp91 ${ }^{\text {phox }}$ (phox, phagocytic oxidase) subunit, known as NOX2, which is expressed in monocytes/macrophages and granulocytes. The phagocytic NADPH oxidase NOX2dependent respiratory/oxidative burst is one of the main sources of $\mathrm{O}_{2}{ }^{\bullet-}$, reaching even $25 \mu \mathrm{M}$ inside a phagolysosome, which is formed from the cell membrane-composed phagosome $(133,326)$. Phagosome formation occurs in $\sim 20 \mathrm{~s}(318)$, after which the microbes containing the phagosome are internalized in the cell, fused with lysosomes to form phagolysosomes where the microbes are destroyed by oxidative burstderived ROS and the action of antimicrobial factors (333).

Cellular responses to stimuli may activate oxidative burst through the calcium-protein kinase $\mathrm{C}\left(\mathrm{Ca}^{2+}-\mathrm{PKC}\right)$ signaling pathway, leading to increased ROS production by NOX2 assembly (described below) at the phagolysosome membrane [reviewed in Ginis and Tauber (108) and Slauch (333)]. Although the NADPH oxidase NOX2 is commonly described in phagocytic cells, it is also expressed in nonphagocytic cells, such as cardiomyocytes (181) and leukemia cells (220). The other nonphagocytic enzymes NADPH oxidase NOX1, NADPH oxidase NOX3, NADPH oxidase NOX4, and NADPH oxidase NOX5, which produce less $\mathrm{O}_{2}^{\bullet-}$ than phagocytic NADPH oxidase $\mathrm{NOX} 2$, use a similar principle in $\mathrm{O}_{2}{ }^{\bullet-}$ production, although the multiunit structure of the enzyme varies [reviewed in Bedard and Krause (19) and Panday et al. (270)].

Nonphagocytic NADPH oxidase NOX1-, NADPH oxidase NOX4-, and NADPH oxidase NOX5-derived ROS play a prominent role in the initiation and progression of cancer through the regulation of cellular signal transduction pathways $(114,159,328,342)$ and through modulation of the growth modulation signaling that supports the autonomous growth of cells, angiogenesis, invasion, and metastasis $(240,260,352)$.

\section{NADPH Oxidase NOX1}

The NADPH oxidase NOX1 complex is composed of the NOX1 subunit (gp91 ${ }^{\text {phox }}$ homologue) (342), NADPH oxidase organizer 1 subunit (NOXO1), which is a homologue of $\mathrm{p} 47^{\text {phox }}$, NADPH oxidase activator 1 subunit (NOXA1), which is a homologue of $\mathrm{p} 67^{\text {phox }}(102), \mathrm{p} 22^{\text {phox }}$ subunit (7), and small GTPase RAC1 subunit (242). NOXO1 colocalizes with NOX1 to resting cell plasma membrane by binding to the phosphatidylinositol (PtdIns) lipids PtdIns 3,5-P2, PtdIns 5-P, and PtdIns 4-P with the Phox homology (PX) domain, hence suggesting both activation and localization roles for NOXO1 (51). NOX1 expression is most prominent in the colon where it is induced by factors representing various cellular effectors that increase both messenger RNA (mRNA) and protein expression, thereby stimulating $\mathrm{O}_{2}{ }^{\bullet-}$ production. The NOX1 inducers include various RTK ligands, such platelet-derived growth factor (PDGF) (188), serine protease urokinase plasminogen activator (UPA) (237), peptide hormone angiotensin II (Ang II) (188), and cytokines-for example, bone morphogenic protein 4 (BMP4) (337) and interferon gamma (IFN- $\gamma)(88,183)$ (Fig. 1C).

The assembly and activation of the NADPH oxidase complex NOX1 are initiated by NOXO1 phosphorylation at Ser154 by cAMP-stimulated protein kinase A (PKA) (72, 388). Phorbol 12-myristate 13-acetate (PMA), a potent mitogen, has been demonstrated to activate PKC, which in turn may induce the phosphorylation of NOXO1 at Thr341/Ser154, resulting in the interaction of NOXO1 with NOXA1 and the simultaneous increase of $\mathrm{O}_{2}^{\bullet-}$ production (388). The prolinerich region at the $\mathrm{N}$-terminal end of NOXA1 can bind to SRC downstream targets tyrosine kinase substrate with 4 SRC homology 3 (SH3) domains (TKS4) and tyrosine kinase substrate with $5 \mathrm{SH} 3$ domains (TKS5), which enhances NOXA1 binding to NOX1 and causes consequent localization to invapodia and increased ROS production (106).

The extracellular stimulus-induced mitogenic RAS-MEKERK and PKC-PKA pathways control the hyperactivation of radical production through increased NOX1 gene expression and phosphorylation of NOXA1 at Ser282 and Ser172, thereby decreasing the affinity of RAC1 for $\operatorname{NOX} 1(5,182)$ (Fig. 1D).

\section{A. NADPH oxidase NOX1 in tumorigenesis}

The role of the NADPH oxidase NOX1 in cell transformation is indisputable. Overexpression of this enzyme has been shown to promote the carcinogenic conversion of NIH3 T3 fibroblasts, causing morphological changes, increased anchorage-independent growth, and increased tumor formation in vivo $(240,342)$. However, it has been suggested that NADPH oxidase NOX1 plays a crucial role only in the initiation of tumorigenesis, as its expression is limited to the early stages of carcinogenesis and is downregulated in advanced cancers, excluding colon cancer $(101,240)$. NADPH oxidase NOX1-induced tumorigenesis is mediated primarily by a signal transduction pathway composed of mutated K-RASV12stimulated p38MAPK, 3-phosphoinositide-dependent protein kinase-1 (PDPK1), and PKC delta (PKC $\delta$ ) (Fig. 2A).

Phosphorylated p38MAPK activates PDPK1, a serine/threonine kinase that functions upstream of phosphatidylinositol-4,5bisphosphate 3-kinase-protein kinase A (PI3K-PKA/AKT) pathway, p70S6 kinase, p90S6 kinase, p21-activated kinase (PAK1), and PKC. Activated PDPK1 phosphorylates PKC $\delta$ at Thr505 allowing the direct interaction of $\mathrm{PKC} \delta$ with the $\mathrm{SH}$

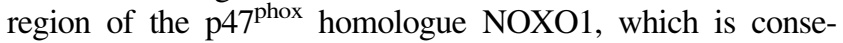
quently phosphorylated at Ser348 and Ser379. Activation of the p $47^{\text {phox }}$ homologue NOXO1 induces NADPH oxidase NOX1 complex assembly and the initiation of ROS production, resulting in increased anchorage-independent growth in vitro and increased tumorigenesis in vivo $(196,278)$ (Fig. 2A).

Although the function of $\mathrm{p} 38 \mathrm{MAPK}$ in tumorigenesis is still under debate, the increased p38MAPK phosphorylation in breast, lung, and thyroid cancer patients correlates with lymph node metastasis, tamoxifen resistance, and poor prognosis (367). Paradoxically, several studies have reported tumor suppressor properties of p38MAPK, which correlate to the ROS concentration in the cells $(76,214$, 367 ), therefore suggesting that the role of p38MAPK in tumorigenesis may depend on upstream/downstream mediators and their function. 

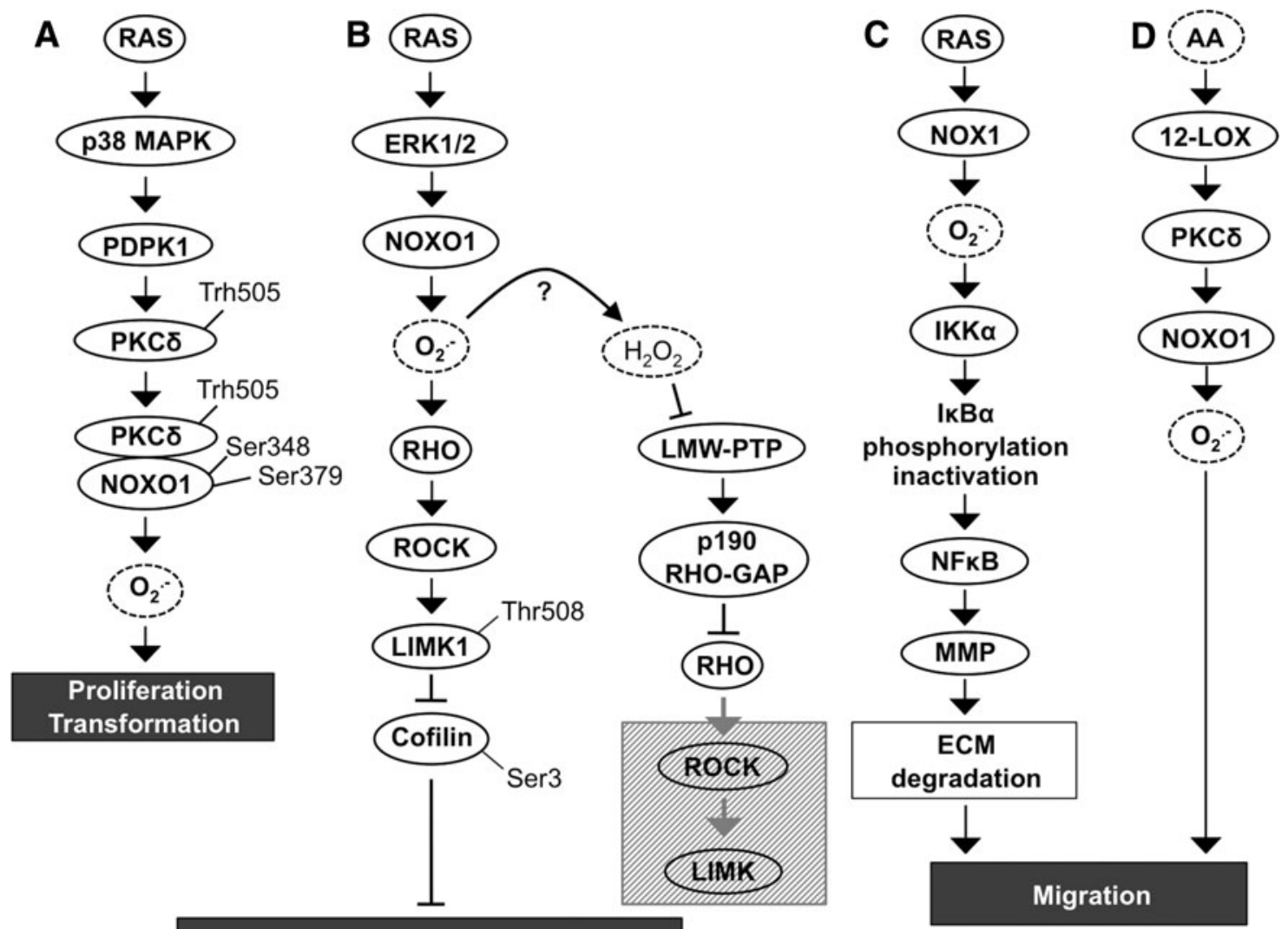

Inhibition of actin depolymerization

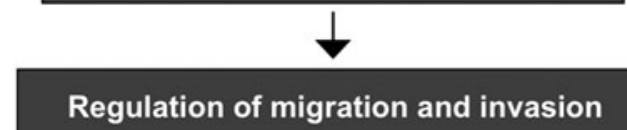

FIG. 2. RAS induces the proliferation and migration of cancer cells via NOX1. (A) The RAS-p38MAPK signaling pathway induces $\mathrm{PKC} \delta$ phosphorylation at Thr505, which causes consequent $\mathrm{PKC} \delta$-NOXO1 dimerization and phosphorylation of NOXO1 at Ser348 and Ser379. NADPH oxidase NOX1 produces $\mathrm{O}_{2}{ }^{--}$thereby stimulating cancer cell migration. (B) RAS-ERK1/2 induced NOXO1 activation and increased $\mathrm{O}_{2}{ }^{\bullet-}$-stimulated signaling downstream to the RHO-ROCKLIMK1 pathway that then inhibits cofilin by phosphorylation at Ser3 and consequently impacts actin depolymerization. $\mathrm{H}_{2} \mathrm{O}_{2}$ produced after activation of NOXO1 may inactivate phosphotyrosine phosphatase LMW-PTP. Consequent increased expression of p190 RHO GAP enhances GTP removal from RHO small GTPase downregulating the downstream ROCKLIMK1 pathway. (C) RAS-induced signaling through IKK $\alpha-\mathrm{NF} \kappa \mathrm{B}$ induces local cancer cell migration by MMP activation and ECM degradation. (D) Migration is also stimulated by arachidonic acid and 12-lipoxygenase-induced PKC $\delta$ signaling that activates NOXO1 and increases $\mathrm{O}_{2}{ }^{\bullet-}$ production. ECM, extracellular matrix; GAP, small GTPase activator protein; IKK $\alpha$, inhibitor of nuclear factor kappa-B kinase subunit $\alpha$; LIMK, LIM kinase; LMW-PTP, low-molecular-weight phosphotyrosine phosphatase; MMP, matrix metalloproteinase; $\mathrm{NF} \kappa \mathrm{B}$, nuclear factor kappa-light-chain-enhancer of activated B cells; PTP, protein tyrosine phosphatase; ROCK, RHO-associated, coiled-coil-containing protein kinase.

In addition to the transformation effect in colon, breast, prostate, lung, and in ovarian cancer cells $(5,102,240,342)$, the increased tumorigenicity of NADPH oxidase NOX1 is mediated through angiogenic stimuli by the upregulation of vascular endothelial growth factor (VEGF) expression and the phosphorylation of VEGF receptors VEGFR-1 and VEGFR-2 (12). VEGF, an efficient promoter of endothelial cell migration in angiogenesis, has been demonstrated to stimulate breast carcinoma invasion utilizing chemokine receptor C-X-C chemokine receptor type 4 (CXCR4) affinity toward stromal-derived factor 1 (SDF-1) (14). Notably, increased ROS production by RAS-MEK1/2-ERK1/2-NADPH oxidase NOX1 upregulates small GTPase RHO and its direct downstream target RHO-associated, coiled-coil-containing protein kinase (ROCK) (Fig. 2B). Several reports have demonstrated ROCK-driven phosphorylation of LIM kinase (LIMK) that then through downstream cofilin, an actin binding protein, regulates disassembly of actin filaments. Phosphorylation of LIMK1 at Thr508 causes inactivation of cofilin by Ser3 phosphorylation, inhibition of actin depolymerization, and accumulation of actin fibers. Nonphosphorylated cofilin resides in the cellular protrusions of migrating cancer cells, whereas Ser3-phosphorylated inactive cofilin is distributed throughout the cytoplasm, thereby affecting cellular motility. Interestingly, $\mathrm{H}_{2} \mathrm{O}_{2}$ can inactivate low-molecular-weight phosphotyrosine phosphatase (LMWPTP), which results in increased p190RHO-small GTPase activator protein (GAP) production and subsequent inactivation of RHO-ROCK-LIMK pathway (30, 212, 268, 281, 300, 330) (Fig. 2B). 
RAS regulates cancer cell migration also through the inhibitor of nuclear factor kappa-B kinase subunit $\alpha(\mathrm{IKK} \alpha)$ nuclear factor kappa-light-chain-enhancer of activated B cell $(\mathrm{NF} \kappa \mathrm{B})$ pathway. NADPH oxidase NOX1 causes increased activity of IKK $\alpha$, which phosphorylates $\mathrm{I} \kappa \mathrm{B} \alpha$ (nuclear factor of kappa light polypeptide gene enhancer in B cell inhibitor $\alpha$ ), an inhibitor of $\mathrm{NF} \kappa \mathrm{B}$. Phosphorylation induces the degradation of $\mathrm{I} \kappa \mathrm{B} \alpha$, increasing $\mathrm{NF} \kappa \mathrm{B}$ activity, which in turn augments matrix metalloproteinase 9 (MMP9) expression and initiates extracellular matrix (ECM) degradation with consequent cancer cell invasion (330). Therefore, NOX1driven invasion of cancer cells is based on the disruption of focal adhesions and increased expression of ECM-degrading enzymes, allowing cells to migrate locally and invade through ECM to initiate metastasis (Fig. 2C). In addition, the arachidonic acid (AA)-induced AA-12-lipoxygenase (LOX)PKC-NOX1 pathway has been shown to decrease $\alpha_{2} \beta_{1}$ integrin expression in cell membranes, eventually causing the loss of actin stress fibers and $\alpha_{2} \beta_{1}$ integrins in cell membranes and therefore abrogating focal adhesions. The NADPH oxidase NOX1-stimulated local cancer cell migration along collagen I fibers depends on the NADPH oxidase NOX1induced oxidative burst caused by AA-activated 12-LOX, which results in phosphorylation of PKC. Activated PKC phosphorylates the NOXO1 subunit, stimulating NOX1 assembly and thus increasing ROS production (308, 330) (Fig. 2D).

\section{NADPH Oxidase NOX2}

\section{A. NADPH oxidase NOX2 complex formation}

NADPH oxidase NOX2, commonly referred by the catalytic subunit NOX2, is the most thoroughly characterized member of the NADPH oxidase family. Although NOX2 is mainly expressed in inflammatory cells involved in various biological functions, for example, in the host defense against invading microbes, it is frequently observed in tissues, such as cardiac muscle $(168,181)$. Signal transduction studies have shown that NADPH oxidase NOX2 complex formation commits small GTPase proteins and G protein-coupled receptor (GPCR), key signaling molecules mediating the extracellular stimuli into the intracellular signal transduction network, as a subunit of NADPH oxidase and as an activator of NADPH oxidase complex formation, respectively.

In the resting-state cell, NADPH oxidase NOX2 units are organized into three different groups: (i) NOX2/gp $91^{\text {phox }}$ and $\mathrm{p} 22^{\text {phox }}$, which form a membrane-bound flavocytochrome b588 (cyt b588) (280); (ii) $\mathrm{p} 47^{\text {phox }}, \mathrm{p} 67^{\text {phox }}$, and $\mathrm{p} 40^{\text {phox }}$, which are located in the cytosol as a heterotrimer attached to each other at their C-terminal ends $(95,203)$; (iii) the third component is small GTPase RAC1 (expressed in macrophages) (287) or RAC2 (expressed in granulocytes) (86, 130) (Fig. 3A). Phosphorylation of $\mathrm{p} 47^{\text {phox }}$ initiates conformational changes in the $\mathrm{p} 47^{\text {phox }}$ structure and the assembly of the NADPH oxidase NOX2 multistructure by transporting the $\mathrm{p} 47^{\text {phox }}-\mathrm{p} 67^{\text {phox }}$ $\mathrm{p} 40^{\text {phox }}$ heterotrimer to the cell membrane $(85,140)$.

At the cell membrane, the heterotrimer is organized to enable the assembly of activated NADPH oxidase and the oxidative burst in the following steps: (i) the $\mathrm{p} 47^{\text {phox }}$ SH3 domain interacts with the cyt b588 subunit p22 $2^{\text {phox }}$ C-terminal proline-rich region (343); (ii) $\mathrm{p} 67^{\text {phox }}$ binds to the cyt b588 gp91 ${ }^{\text {phox }}$ subunit NADPH binding domain and
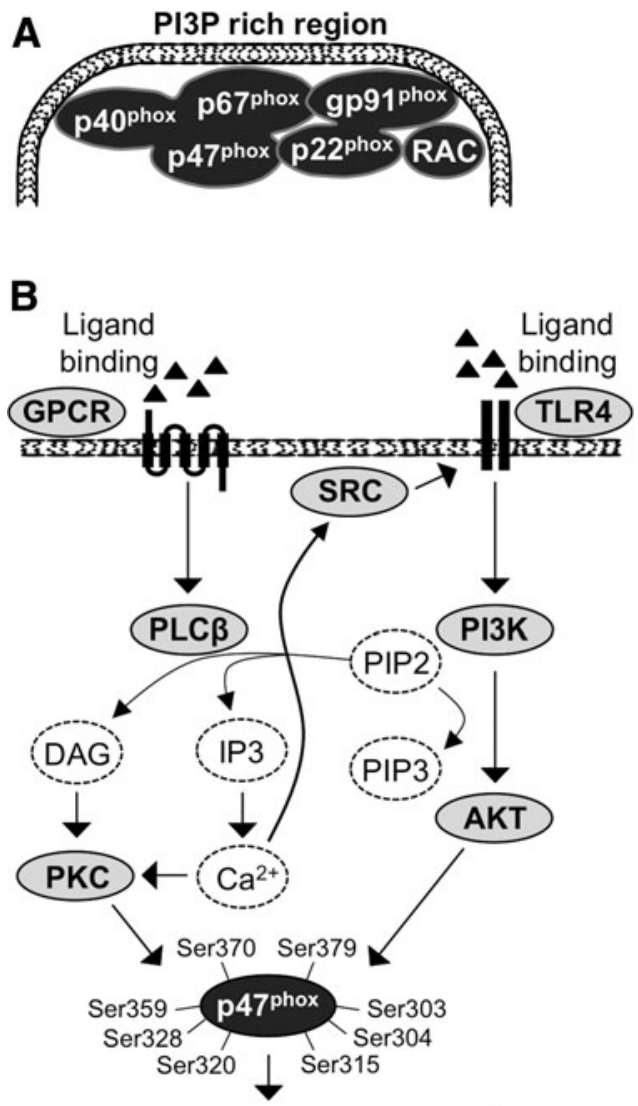

Initiation of NADPH oxidase NOX2 assembly

FIG. 3. Activation of NADPH oxidase NOX2 assembly. (A) NADPH oxidase NOX2 is composed of a p40 $40^{\text {phox }}$ p6 $7^{\text {phox }}-\mathrm{p} 47^{\text {phox }}$ heterotrimer and a p22 $2^{\text {phox }}$-gp91 $91^{\text {phox }}$ dimer that is activated at the cell membrane by association of small GTPase RAC into the complex. (B) GPCR activation stimulates PLC $\beta$-DAG/IP3-Ca ${ }^{2+}-\mathrm{PKC}$ signaling that phosphorylates $\mathrm{p} 47^{\mathrm{phox}} \cdot \mathrm{Ca}^{2+}$ may alternatively phosphorylate the SRC oncogene followed by activation of the TLR4-PI3K-AKT pathway, which further stimulates $\mathrm{p} 47^{\mathrm{phox}}$ subunit phosphorylation. $\mathrm{Ca}^{2+}$, calcium; $\mathrm{Ca}^{2+}-\mathrm{PKC}$, calcium-protein kinase $\mathrm{C}$; DAG, diacylglycerol; GPCR, G protein-coupled receptor; IP3, inositol triphosphate; PI3K, phosphatidylinositol-4,5bisphosphate 3-kinase; PLC, phospholipase C; TLR4, Tolllike receptor 4 .

functions as a regulator of oxidase activity (211); (iii) $\mathrm{p} 40^{\text {phox }}$ binds to PI3P at PI3P-rich membrane regions, thus strengthening the interaction of the membrane and NAPDH oxidase complex in the presence of phosphorylated PI3K $(359,360)$ (Fig. 3A); and (iv) the final activation of the NADPH oxidase NOX2 complex occurs by the interaction of activated GTP-bound RAC with the p6 $7^{\text {phox }} \mathrm{N}$-terminal region, enhancing gp91 subunit $\mathrm{O}_{2}^{\bullet-}$ production (176). RAC is an important regulatory factor in electron transfer from NADPH to flavin adenine dinucleotide (FAD) and consequently from FAD to heme groups in the gp91 ${ }^{\text {phox }}$ catalytic center (75). More specifically, the association of small GTPase RAC activates the two-step electron transfer reaction: (i) electron transfer from NADPH to FAD, which results in the formation of $\mathrm{NADP}^{+}$and $\mathrm{FADH}_{2}$ and (ii) 
electron transfer to cyt b588-associated heme and molecular oxygen, resulting in the formation of $\mathrm{H}^{+}$and $\mathrm{O}_{2}{ }^{--}$(75).

In response to stimuli, the activated phagocytic NOX2 (gp91 ${ }^{\text {phox }}$ unit of the complex), which contains one FAD and two nonidentical heme molecules, gradually catalyzes $\mathrm{O}_{2}$ to $\mathrm{O}_{2}{ }^{\bullet-}$. The oxidative burst is initiated by electron transfer from NADPH to FAD, followed by electron transfer to the heme group, which, in turn, reduces $\mathrm{O}_{2}$ to $\mathrm{O}_{2}{ }^{\bullet-}(19,64)$. Activation of the oxidative burst is a multistep process stimulated by various signals, such as bacterial lipopolysaccharide (LPS), inflammatory cytokines, and platelet-activating factor (PAF), which can stimulate the activation of GPCR and Toll-like receptor 4 (TLR4) (134, 371). Ligand binding induces a conformational change in GPCR, eventually promoting the GDP switch to GTP, causing the activation of G proteins associated with GPCR and initiating the downstream signaling cascade (349).

TLR4 belongs to the type- 1 transmembrane receptor family that is important in activation of NADPH NOX2 complex assembly (45) (Fig. 3B). In addition, TLR4 stimulates NF $\kappa \mathrm{B}$ signal transduction pathway that activates tumor promoting molecules, such as VEGF-A, cyclooxygenase 2 (COX2), interleukins IL-6 and IL-8, and MMP9, which all increase cancer cell survival, immune escape, and increased metastasis (295).

Activation of GPCR stimulates the Gq heterotrimeric G protein subunit, which initiates phospholipase $\mathrm{C} \beta$ (PLC $\beta$ )driven hydrolysis of membrane-bound phosphatidylinositol (4,5)-biphosphate (PIP2), resulting in the synthesis of diacylglycerol (DAG) and inositol triphosphate (IP3). DAG recruits PKC to the cell membrane, and IP3 promotes calcium $\left(\mathrm{Ca}^{2+}\right)$ channel opening with a consequent temporal intracellular increase of $\mathrm{Ca}^{2+}$, which further enhances the activation of PKC, a crucially important kinase in the initiation of NADPH oxidase complex assembly $(24,277)$ (Fig. 3B). Several studies have demonstrated that increased intracellular $\mathrm{Ca}^{2+}$ uptake activates the phosphorylation of the proto-oncogene SRC.

The SRC family of protein tyrosine kinases, which comprises nine members (SRC, YES, LYN, YRK, BLK, FYN HCK, FGR, and LCK), interacts with RTKs and GPCRs, mediating their signaling to downstream networks. As mentioned above, the activation of SRC increases the phosphorylation of TLR4, which enhances PI3K-AKT pathway signal transduction, causing the phosphorylation of the NADPH oxidase regulatory subunit $\mathrm{p} 47^{\mathrm{phox}}$ together with $\mathrm{PKC}$ at serines 303, 304, 315, 320, 328, 359, 370, and 379 (45, 57, 92, $140,253,270,334)$. More specifically, PI3K class I that is activated by downstream signaling from GPCRs and RTKs uses PIP2 as a precursor for the production of phosphatidylinositol (3,4,5)-triphosphate (PIP3), which then activates AKT immediately downstream of PI3K.

Interestingly, PI3K has been suggested to be activated simultaneously with PLC, resulting in simultaneous catalysis of PIP2 and consequent synthesis of PIP3, DAG, and IP3 (256, 294, 316) (Fig. 3B).

\section{NADPH Oxidase NOX3}

Similar to NOX1 and NOX2, NOX3 contains the catalytic subunit gp91 ${ }^{\text {phox }}$. The oxidative burst by NADPH oxidase NOX3 is stimulated by regulatory subunits p47phox and p67phox, or by subunits NOXO1 and NOXA1, thus showing compatibility for both NOXO and phox proteins. In addition, p22 phox is required for $\mathrm{O}_{2}^{\bullet-}$ production $(52,358)$. NOX3 is mostly expressed in the inner ear, fetal kidney, liver, lung, and spleen but is detected also at low mRNA levels in adult colon tissue $(50,269)$, suggesting a role for NOX3 in ontogenesis, findings that were corroborated by a study showing a crucial role of NADPH oxidase NOX3 in otoconial morphogenesis in the inner ear. The authors hypothesized that otoconin bound to membrane structure phospholipids undergoes NADPH oxidase NOX3-catalyzed conformational change. In the absence of NADPH oxidase NOX3, otoconin morphogenesis is defective. Abnormalities in otoconia may cause symptoms similar to those of vertigo (269).

Although the functional role of NADPH oxidase NOX3 is reported only in otoconia morphogenesis, there may be an association with the primitive cellular phenotype in general because NOX3 mRNA expression is moderately augmented in undifferentiated ovarian teratocarcinoma and adenocarcinoma cancer cells (50).

\section{NADPH Oxidase NOX4}

NOX4 expression and function are linked to primary cell transformation, fibrosis, and cardiovascular diseases $(25,47,65$, $156,389)$. The NOX4 isoform of NADPH oxidase comprises p22 $2^{\text {phox }}$, polymerase delta-interacting protein 2 (209), and TKS5 (74). According to a recent work, small GTPase RAC1 can associate with the NADPH oxidase NOX4 complex, but it is not needed for full NOX4 activation (236). The most striking difference between NOX4 and other isoforms is the high concentration of $\mathrm{H}_{2} \mathrm{O}_{2}$ produced by the enzyme.

Although $\mathrm{H}_{2} \mathrm{O}_{2}$ synthesis by NADPH oxidase NOX4 has not been completely characterized, it has been suggested that the outer membrane E-loop may produce protons $\left(\mathrm{H}^{+}\right)$, which then permit increased spontaneous dismutation of $\mathrm{O}_{2}{ }^{\bullet-}$ to $\mathrm{H}_{2} \mathrm{O}_{2}$ (345). The same kinds of E-loop structures, although shorter, exist also in NOX1 and NOX2 NADPH oxidases, which, however, do not possess similar dismutase activity (345). Therefore, further studies are required to dissect whether $\mathrm{O}_{2}{ }^{\bullet-}$ catalysis to $\mathrm{H}_{2} \mathrm{O}_{2}$ is purely spontaneous, based on the NADPH oxidase NOX4-associated subunit or enzyme.

\section{A. NADPH oxidase NOX4 in tumorigenesis}

Increased NOX4 expression has been frequently connected to cell transformation. Recent studies suggested that in acute myeloid leukemia (AML) cells, mutated FMS-like tyrosine kinase 3 with internal tandem duplications (FLT3-ITDs) increases the synthesis of transcription factor signal transducer and activator of transcription 5 (STAT5), which binds to the $N O X 4$ promoter region at IFN- $\gamma$ activated sequence elements, activating NOX4 gene transcription. The FLT3-ITD oncogene also induces the phosphorylation of PI3K-AKT that then activates the $\mathrm{p} 22^{\mathrm{phox}}$ subunit with consequent NADPH oxidase NOX4 assembly.

NADPH oxidase NOX4-derived $\mathrm{O}_{2}^{\bullet-}$ and $\mathrm{H}_{2} \mathrm{O}_{2}$ then contribute to the inactivation of protein tyrosine phosphatase, receptor type $\mathrm{J}$ (PTPRJ, also known as DEP-1), a transmembrane PTP that negatively regulates FLT3 signaling activity, and the transformation of primary cells $(155,156)$. Hence, NADPH oxidase NOX4-derived inactivation of PTPRJ could explain increased FLT3 activation and the consequent transformation of hematopoietic cells. Furthermore, FLT3-ITD overexpression has been shown to increase 
DNA damage, such as DNA oxidation modification and double-strand breaks; this damage contributes to the initiation and progression of carcinogenesis, resulting in an aggressive cancer cell phenotype and enhanced drug resistance with consequent disease relapse. Mechanistically, p22 $2^{\text {phox }}$ has been suggested to mediate FLT3-ITD-stimulated ROS production by activating NOX4 in the nuclear membrane of leukemia cells, thereby causing increased nuclear ROS contents and consequent DNA damage $(155,156,338)$. Additional evidence for the tumor-promoting role of NADPH oxidase NOX4 was offered by a study demonstrating NADPH oxidase NOX4 increased expression of cyclin-dependent kinase $1(C D K 1)$ and cell division cycle 25C/M-phase inducer phosphatase $3(C D C 25 c)$, both of which promote cell cycling, anchorage-independent growth, and melanoma tumorigenesis in vivo (389).

Based on the previous observations, the growth stimulatory function of NADPH oxidase NOX4 is not limited to hematopoietic cells, but NADPH oxidase NOX4 can support also renal tumorigenesis via increased nuclear accumulation of hypoxia-inducible factor $2 \alpha$ (HIF- $2 \alpha$ ), therefore affecting phenotypic morphogenesis, colony formation, invasion, and in vivo tumor growth (114). In thyroid cancer models, NOX4 expression has been detected in papillary thyroid tumors and was shown to be stimulated by RAS and adenovirus E1A oncogenes, suggesting that NADPH oxidase NOX4 is a mediator of oncogene action $(34,374,375)$. In addition, in a recent study, NOX4 expression was observed in thyroid cancer and in papillary thyroid cancer (PTC)-derived mesenchymal stem/stromal cells (MSCs) (275), thus indicating a paracrine role for NADPH oxidase NOX4 in thyroid function.

The tumor stroma microenvironment plays a crucial role in the initiation and development of tumors. The bidirectional paracrine effect activates tumor-associated fibroblasts to myofibroblasts, which then stimulate epithelial cell proliferation, migration, and metastasis (35). Transforming growth factor $\beta(\mathrm{TGF} \beta)$ is a well-known regulator of cancer cell growth and stimulator of fibrotic reaction in several pathological conditions, such as renal fibrosis, liver cirrhosis, myocardial sclerosis, idiopathic pulmonary fibrosis, and desmoplastic reaction in advanced metastatic cancers. In general, TGF $\beta$ ligand binding to the TGF $\beta$ R2/TGF $\beta$ R1 heterodimer activates a cascade in which TGF $\beta$ R 2 is phosphorylated first, followed by the phosphorylation of TGF $\beta$ R1.

The activated receptor dimer phosphorylates the SMAD2/ SMAD3 dimer, allowing the association of SMAD4 into the complex. The SMAD complex then translocates to the nucleus, binds to DNA, and stimulates target gene expression. Interestingly, in the early phase of carcinogenesis, TGF $\beta$ functions as a tumor suppressor and as a tumor promoter at the end phase of carcinogenesis. This "TGF $\beta$ paradox" is caused by the modification of $\mathrm{p} 53$. Wild-type $\mathrm{p} 53$, which binds to the nuclear SMAD complex, inhibits growth together with tumor suppressor $\mathrm{p} 63$, whereas mutated $\mathrm{p} 53$, by binding to the SMAD complex, does not suppress growth and, additionally, inhibits p63 binding to SMAD complex (40).

Recently, TGF $\beta$ has been suggested to promote cancer cell migration through increased ROS production. The paracrine effect of human mammary MCF-7 cells has been demonstrated to activate the TGF $\beta$-SMAD3 signal transduction pathway in mammary epithelial and stromal cells, inducing NOX4 expression and the consequent phosphorylation of focal adhesion kinase (FAK). FAK signaling downstream through the SRC and PI3K-AKT pathways is responsible for cell immobilization by attaching cells to ECM. Thus, the activation of FAK initiates local cancer cell migration and metastasis $(27,350)$.

Fibroblast differentiation to myofibroblasts is the cornerstone in cancer progession. TGF $\beta$ contributes to fibrotic signaling by activating RHO small GTPase and the downstream RHO-associated kinase ROCK. Activation of ROCK phosphorylates POLDIP2, which increases NOX4 gene and protein expression with consequent augmented ROS production. NADPH oxidase NOX4 has been demonstrated to be a mediator of TGF $\beta$-RHO-ROCK-stimulated c-Jun N-terminal kinase (JNK) activation, which then increases myofibroblast differentiation-related gene expression-for example, insulinlike growth factor binding protein 3 (IGFBP3) and alpha smooth muscle cell acting expression-and morphological changes (216) (Fig. 4A).

\section{NADPH Oxidase NOX5}

There are two types of NOX5 genes; NOX5-S and NOX5-L (96). The latter has five splice variants; NOX $5 \alpha, \operatorname{NOX} 5 \beta$, NOX $5 \gamma, \operatorname{NOX} 5 \delta$, and NOX $5 \varepsilon$, which functional significance is not completely characterized. Although all five splice variants are expressed in vascular endothelial and smooth muscle cells $(20,154)$, the expression of the splice variants shows also tissue-specific expression pattern: NOX5 $\alpha$ is mainly expressed in the spleen, $N O X 5 \beta$ is mainly expressed in the testis, and

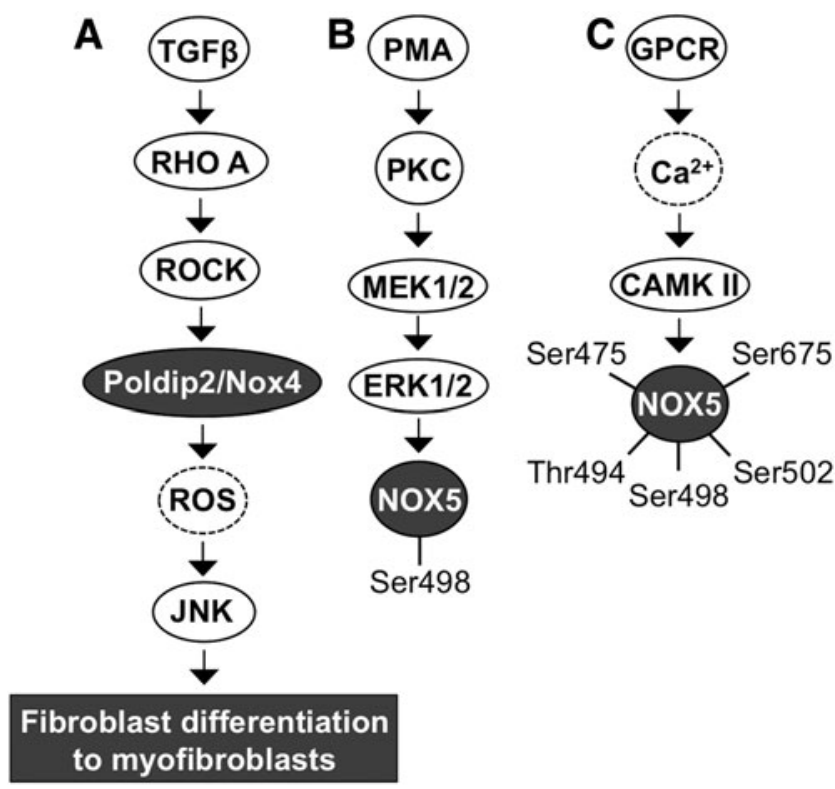

FIG. 4. NOX4 and NOX5 activation. (A) TGF $\beta$-driven activation of RHO-ROCK signaling increases NOX4 synthesis and consequent ROS production. (B) Mitogen stimulus caused by the PMA-activated PKC-MEK1/2-ERK1/2 signaling cascade stimulates NOX5 phosphorylation at Ser498. (C) GPCR activation increases $\mathrm{Ca}^{2+}$ influx, which then increases CAMKII and PKC $\alpha$ activation and consequently increases NOX5 phosphorylation at Ser475, Thr494, Ser498, Ser502, and Ser675. CAMKII, calcium/calmodulin-dependent kinase II; GPCR, G protein-coupled receptor; JNK, C-Jun Nterminal kinase; PMA, phorbol 12-myristate 13-acetate; ROS, reactive oxygen species; TGF $\beta$, transforming growth factor $\beta$. 
truncated $N O X 5 \varepsilon$ is mainly expressed in esophageal cancer $(15,50,94,96)$. Isoforms have been suggested to contribute to coronary artery disease development, myocardial infarction, fetal ventricular septal defect, cancer, and irradiation-derived DNA damage in human primary fibroblasts and peripheral blood mononuclear cells $(96,376)$. In cells, NADPH oxidase NOX5 is located mostly in the endoplasmic reticulum where the enzyme mainly synthesizes $\mathrm{O}_{2}^{\bullet-}$ but has also been reported to produce $\mathrm{H}_{2} \mathrm{O}_{2}(10,96,139,369)$.

Structurally, NADPH oxidase NOX5 is composed of six transmembrane domains carrying two heme molecules, a cytoplasmic N-terminus domain carrying four $\mathrm{Ca}^{2+}$ binding sites, and a cytoplasmic C-terminus domain carrying NADPH and FAD binding sites (50). On activation by $\mathrm{Ca}^{2+}$ binding to the N-terminal domain, the C-terminal domain-bound NADPH releases electrons to FAD and further to heme molecules, resulting in $\mathrm{O}_{2}^{\bullet-}$ production (96). Although NOX5 isoform activation is dependent on $\mathrm{Ca}^{2+}$, it is independent of the $\mathrm{p} 40^{\text {phox }}, \mathrm{p} 47^{\text {phox }}, \mathrm{p} 67^{\text {phox }}$, and $\mathrm{p} 22^{\text {phox }}$ cytosolic subunits. $\mathrm{Ca}^{2+}$ binding to N-terminal EF motif results in conformational changes, enabling the interaction between the EF motif and catalytic domain of the NOX5 subunit of NADPH oxidase $(16,48,96)$.

$\mathrm{Ca}^{2+}$-cAMP response element-binding protein (CREB)and $\mathrm{Ca}^{2+}$-PAF-ERK1/2-phospholipase A2 (PLA A $_{2}$-STAT5mediated signaling stimulate NOX5 mRNA synthesis. $\mathrm{Ca}^{2+}$-dependent activation of $\mathrm{PKC} \alpha$ and $\mathrm{PKC} \varepsilon$ causes the phosphorylation of NOX5 at Ser490, Thr494, and Ser498. Interestingly, $\mathrm{PKC} \delta$ seems to inhibit the production of NADPH oxidase NOX5-derived $\mathrm{O}_{2}{ }^{\bullet-}$. However, the underlying mechanism causing the different effects of $\mathrm{PKC}$ isoforms is not well characterized but could be related to the regulation of $\mathrm{Ca}^{2+}$ influx or activation of $\mathrm{Ca}^{2+}$-related kinases.

PMA, demonstrated to activate MEK1/2-ERK1/2 kinasedriven phosphorylation of NOX5 at Ser498 without involving $\mathrm{Ca}^{2+}$ signaling, is necessary but not sufficient for NADPH oxidase $\mathrm{NOX} 5 \mathrm{O}_{2}^{\bullet-}$ production, thereby suggesting the involvement of other signaling routes (Fig. 4B). Indeed, direct interaction between calcium/calmodulin-dependent kinase II (CAMKII) and NOX5 results in NOX5 phosphorylation at Ser475, Ser498, Ser502, Ser675, and Thr494 (48, 94, 271, 272) (Fig. 4C).

\section{A. NADPH oxidase NOX5 in tumorigenesis}

In gastroesophageal cancer, the activation of truncated form of NOX5, NOX5e, is achieved by phosphatidylinositolspecific phospholipase C (PI-PLC), which releases IP3 and DAG. Interestingly, the signaling is mediated through ERK2, but not through ERK1, leading to full NOX5 $\varepsilon$ activation and ROS production (138). Another mechanism underlying how NOX5 $\varepsilon$ is activated in gastroesophageal cancer involves an increased intracellular $\mathrm{Ca}^{2+}$ concentration that activates small GTPase RHO and the downstream kinase ROCK2. ROCK2, but not ROCK1, then increases NOX5 $\varepsilon$ expression and $\mathrm{H}_{2} \mathrm{O}_{2}$ production (139).

Other signaling routes that upregulate and activate NOX5 are initiated by acid conditions or the IL- 2 inflammatory cytokines, which activate the PAF-MEK-ERK-cytosolic phospholipase $\mathrm{A}_{2} \quad\left(\mathrm{cPLA}_{2}\right)$-JAK-STAT5-NOX5 $\alpha / \varepsilon$ cascade in Barrett's esophageal adenocarcinoma cells and in human $\mathrm{T}$ cell leukemia virus type 1 (HTLV-1) transformed adult T cell leukemia cells
(328, 331). Thereby, the signal transduction pathways connected to the activation of NADPH oxidase NOX5 corroborate the role of the oxidase in growth stimulation. Indeed, RNA interference (RNAi) silencing of NOX5 $\alpha$ and the use of $\mathrm{O}_{2}{ }^{\bullet-}$-neutralizing $\mathrm{N}$-acetyl cysteine (NAC) and diphenyleniodonium (DPI) inhibited prostate cancer and leukemia cell proliferation in vitro and tumorigenesis in vivo, demonstrating NOX5 $\alpha$-driven cancer cell growth $(136,328,331)$. NOX5 has been further shown to support growth and cell proliferation via the PDGF-JAK2-STAT pathway in vascular smooth muscle cells, via the $\mathrm{NF} \kappa \mathrm{B}-\mathrm{COX} 2-\mathrm{PGE}_{2}$ pathway in esophageal adenocarcinoma cells, and via the SHP2-tyrosine PO4 pathway in hairy cell leukemia $(96,154)$.

\section{SOD1-3 Family}

The SOD family consists of three isotypes: CuZnSOD (SOD1), MnSOD (SOD2), and EC-SOD (SOD3). SOD1 was first discovered in 1938 from bovine blood (hemocuprein) and from the liver (hepatocuprein) by Mann and Keilin (217), and in 1957 from the brain (cerebrocuprein) by Porter and Folch (290). McCord and Fridovich identified the discovered protein as an enzyme at the end of 1960s when they demonstrated the dismutase reaction and named the protein CuZnSOD (230). The second isotype, MnSOD, of the family was discovered in 1973 by Zimmermann et al. from mitochondria (399), and the third isotype, EC-SOD, was discovered by Marklund et al. from the extracellular space $(222,224)$.

SOD1, SOD2, and SOD3 catalyze the $\mathrm{O}_{2}{ }^{\bullet-}$ conversion to $\mathrm{H}_{2} \mathrm{O}_{2}$ in two half reactions in which active center $\mathrm{Cu}^{2+}$ is first reduced to $\mathrm{Cu}^{+}$and is then oxidized back to $\mathrm{Cu}^{2+}$ in a reduction/oxidation (redox) reaction (Fig. 1B). During the first half reaction, the oxidized form of the enzyme reacts with $\mathrm{O}_{2}{ }^{\bullet-}$, releasing molecular oxygen. In the second half reaction, the reduced form of the enzyme reacts with the second $\mathrm{O}_{2}{ }^{\bullet-}$ and with two protons $\left(\mathrm{H}^{+}\right)$releasing $\mathrm{H}_{2} \mathrm{O}_{2}$.

In the dismutation reaction, the negatively charged substrate $\mathrm{O}_{2}{ }^{\bullet-}$ is guided into the positively charged channel of the enzyme by the electrostatic field created by the His-61, Glu-119, Lys-120, Glu130, Glu-131, and Lys-134 network. Once positively charged lysine amino acids of the network have attracted the substrate into the channel, Arg-141 further directs $\mathrm{O}_{2}{ }^{--}$into the copper-containing active center where the redox reaction of copper occurs. In the first half-reaction, His-61 disassociates from reduced copper and is forced out of the planar structure connecting the His-61 imidazole ring, copper, and zinc, forming a more tetrahedral structure. The geometrical change of the enzyme enables the second half reaction to occur (103).

\section{Copper Zinc SOD, CuZnSOD, SOD1}

Cytosolic SOD1 is a 32-kDa homodimer formed from two catalytically active nondisulfide-linked subunits. Although the stimulation of SOD1 gene expression is not completely characterized, the promoter region has binding sites for specificity protein 1 (SP1), early growth response 1 (EGR-1), and activator protein 1 (AP1) along with upstream enhancer region-located binding sites for $\mathrm{NF} \kappa \mathrm{B}$, nuclear factor (erythroid-derived 2)-like 2 (NRF2), and CCAAT enhancerbinding protein $(\mathrm{C} / \mathrm{EBP})$, possibly indicating putative transcription regulatory mechanisms $(172,276,320)$. SOD1 has been shown to increase PLC-PKC signal transduction that 
opens voltage-gated $\mathrm{Ca}^{2+}$ channels, allowing $\mathrm{Ca}^{2+}$ influx and causing an increased intracellular $\mathrm{Ca}^{2+}$ concentration in human neuroblastoma cells (247) (Fig. 5A). Other neuronal signal pathways that SOD1 activates include muscarinic M1 acetylcholine receptor coupled to $\mathrm{Gq}_{11}$-ERK1/2 and AKT kinase cascades, modulating synaptic transmission, thus suggesting a function for the enzyme in the neuronal microenvironment $(69,246)$ (Fig. 5B).

SOD1 has been connected to the development of amyotrophic lateral sclerosis (ALS), cancer, ischemia, and altered glucose metabolism $(274,297,302)$. The evidence connecting SOD1 and ALS is based on observations suggesting a high number of mutations that affect the ability of the enzyme to increase the risk of ALS development. Hitherto, there are at least 170 known mutations that are linked to ALS. Mice carrying mutant SOD1 show up to 50-fold reduced affinity for zinc located at the active center of the enzyme (131). Therefore, mutations affecting the stability of the SOD1 active center disrupt the normal activity of the enzyme and may even convert the antioxidant function, thereby increasing the oxidative stress (327).

Under reducing conditions, mutant SOD1 can regulate NOX2 activation by stabilizing RAC1 through direct binding to the small GTPase in a redox-insensitive manner, enhancing the NOX 2 complex assembly and ROS production. Both wildtype and mutant SOD1 interact with RAC1, maintaining the small GTPase in the active GTP-bound form. Importantly, the interaction between wild-type SOD1 and RAC1 is disrupted at increased $\mathrm{H}_{2} \mathrm{O}_{2}$ concentrations, thus allowing the hydrolysis of GTP from RAC1, whereas mutant SOD1 lacks redox sensitivity, maintaining the active RAC1-GTP complex, NADPH oxidase NOX2 oxidative burst, and increased production of $\operatorname{ROS}(120,157)$.

Increased $\mathrm{O}_{2}{ }^{--}$and $\mathrm{H}_{2} \mathrm{O}_{2}$ production activates Toll-like receptor 2 (TLR2) and TLR4 signal transduction through IL1 receptor-associated kinase (IRAK), BH3 domain-only, tumor necrosis factor (TNF) receptor-associated factor 6 (TRAF6), and the $\mathrm{I} \kappa \mathrm{B} \alpha-\mathrm{p} 50 / \mathrm{p} 65$ pathway, eventually increasing the proinflammatory cytokine expression characteristic of ALS (174) (Fig. 5C).

\section{A. SOD1 in tumorigenesis}

SOD1 has a diverse effect on cancer cell signal transduction, growth, and survival. Inhibition of SOD1 with the ATN224 small-molecule inhibitor has been suggested to inhibit epidermal growth factor (EGF)- and insulin growth factor (IGF)-stimulated mitogen signal transduction through ERK1/ 2 kinases. Mechanistically, the inhibition of SOD1 maintains the concentration of $\mathrm{H}_{2} \mathrm{O}_{2}$ at levels that are not adequate to inhibit PTPs, thereby allowing the PTP-mediated inactivation of RTK phosphorylation and attenuation of corresponding downstream signal transduction (158). Inhibition of SOD1 by ATN-224 conceivably increases the intracellular $\mathrm{O}_{2}{ }^{\bullet-}$ concentration, which, surprisingly, leads to the inhibition of glutathione peroxidase activity with a consequently increased lethal level of intracellular $\mathrm{H}_{2} \mathrm{O}_{2}$ concentration.

$\mathrm{H}_{2} \mathrm{O}_{2}$ induces the expression of proapoptotic Bcl-2 interacting mediator of cell death (BIM) and BCL2 binding component 3 (PUMA), and phosphorylation of p38MAPK, which then causes decreased antiapoptotic factor myeloid cell leukemia 1 (MCL1) expression. Consequently, ATN-224-
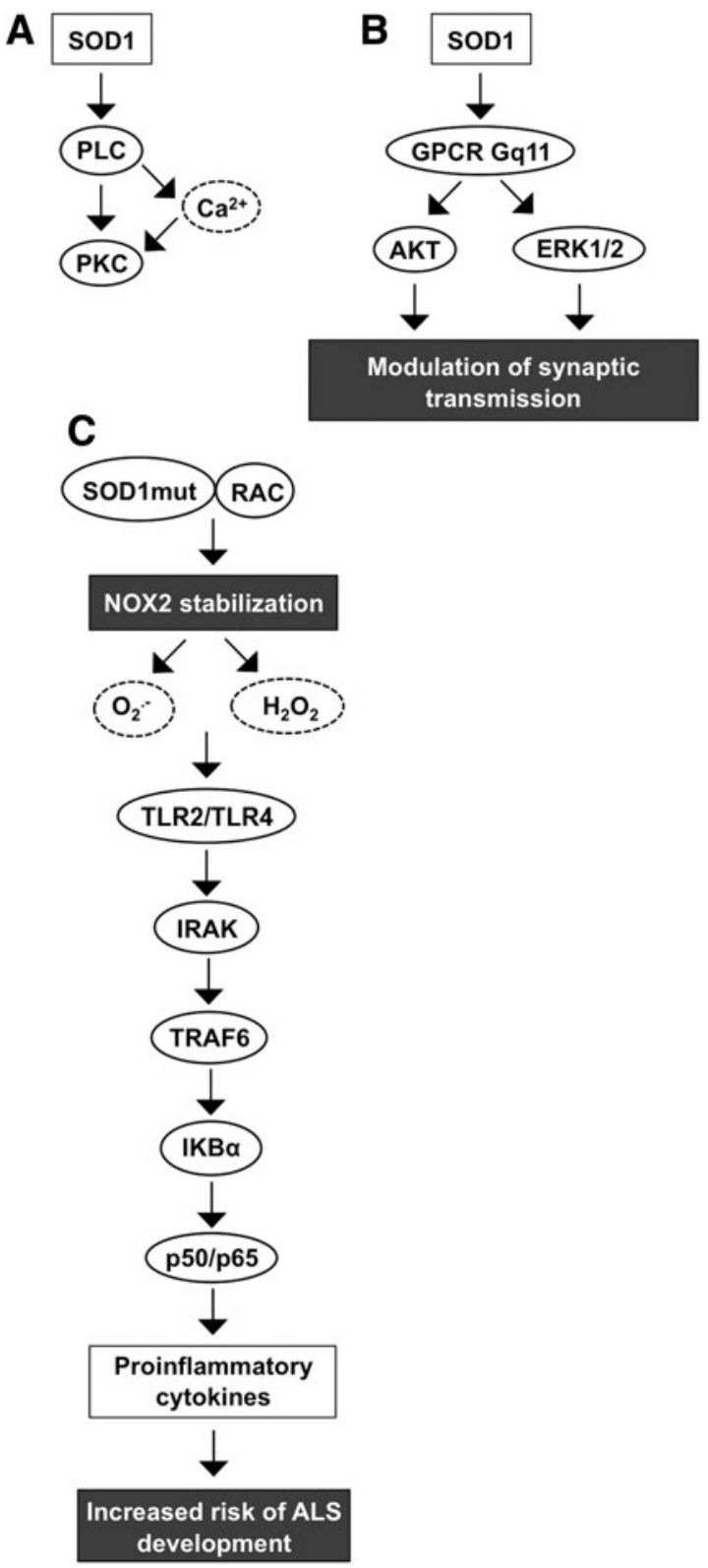

FIG. 5. SOD1 signal transduction. (A) SOD1 activates PLC-Ca ${ }^{2+}$-PKC signaling. (B) Activation of GPCR Gq11 by SOD1 stimulates AKT and ERK1/2 signaling modulating synaptic transcriptions. (C) Mutant SOD1 binds to small GTPase RAC in an redox-insensitive manner causing NADPH oxidase NOX2 stabilization and increased proinflammatory cytokine production through TLR-IRAKTRAF6 signal transduction. Increased cytokine production is a risk factor in ALS development. ALS, amyotrophic lateral sclerosis; IRAK, interleukin-1 receptor-associated kinase; TNF, tumor necrosis factor; TRAF6, TNF receptorassociated factor 6 .

treated cancer cells enter programmed caspase-mediated apoptosis in vitro and in vivo $(110,158)$ suggesting SOD1 as a tumor promoter and a potential novel target for cancer therapy.

SOD1 has been shown to attenuate cellular respiration by increasing aerobic glycolysis in glucose metabolism. In general, glycolysis, which occurs in the cytoplasm, is one of the main ATP synthesis mechanisms in cells together with 
mitochondrial oxidative phosphorylation. In normal cell glycolysis, glucose is metabolized to pyruvate, which is further metabolized to $\mathrm{CO}_{2}$ in the mitochondrial oxidative phosphorylation reaction cascade in the presence of $\mathrm{O}_{2}$. In the absence of $\mathrm{O}_{2}$, pyruvate is metabolized in anaerobic glycolysis, resulting in markedly lower levels of ATP. In cancer cells that have increased glycolysis, and thus increased microenvironmental acidosis, pyruvate is mostly metabolized to lactate in aerobic glycolysis (309). The study of the contribution of SOD1 to glycolysis suggested that Saccharomyces cerevisiae yeast casein kinase 1 gamma $(\mathrm{CK} 1 \gamma)$ homologues Yck1p and Yck2p are stabilized by SOD1 dismutase function.

Functionally, SOD1 utilizes yeast Ynolp NAPDH oxidase-like protein produced $\mathrm{O}_{2}{ }^{\bullet-}$ to increase the cellular $\mathrm{H}_{2} \mathrm{O}_{2}$ concentration in human cells (297), suggesting that mammalian NOX enzymes and SOD1 are signaling partners in the modification of glucose metabolism in cellular respiration increasing aerobic glycolysis. Therefore, SOD1 supports cancer cell growth by maintaining proliferative signal transduction, protecting cells from ROS-derived induction of apoptosis and inducing the metabolic switch from normal respiration to aerobic glycolysis.

\section{Manganese SOD, MnSOD, SOD2}

Tetrameric manganese Mn-II coenzyme containing SOD2 is located almost exclusively in mitochondria, where it converts respiratory chain-produced $\mathrm{O}_{2}{ }^{\bullet-}$ into $\mathrm{H}_{2} \mathrm{O}_{2}$. Each SOD2 monomer contains seven $\alpha$-helices, three antiparallel $\beta$ sheets, and seven connecting structures. The manganesecontaining site of the enzyme is associated with $\mathrm{N}$-terminal helical hairpin domain and $\mathrm{C}$-terminal $\alpha$-helix/ $\beta$-sheet structures. The importance of the enzyme to cellular viability has been demonstrated by studies showing the lethal phenotype of SOD2-knockout mice (201), underlining the critical role of the $\mathrm{O}_{2}{ }^{\bullet-}-\mathrm{H}_{2} \mathrm{O}_{2}$ balance in the mitochondria. The enzyme expression is induced by a high number of factors, including oxidative stress, cytokines, and transcription factors, such as $\mathrm{NF} \kappa \mathrm{B}, \mathrm{SP} 1, \mathrm{CREB}$, and different members of the forkhead box family (FOXO) $(58,218,385)$.

Inhibition of the $\mathrm{NF} \kappa \mathrm{B}$ signal transduction pathway by the kinesin spindle protein inhibitor SB715992 (1 nM concentration) in human multiple myeloma cells results in reduced SOD2 expression and induces cell death in $24 \mathrm{~h}$ (335), therefore confirming the role of $\mathrm{NF} \kappa \mathrm{B}$ in SOD2 stimulation and suggesting a growth-supportive role for the enzyme. Indeed, overexpression of miR146a downregulated SOD2, reduced human epithelial ovarian cancer cell proliferation, increased apoptosis, and increased sensitivity to chemotherapy (67). Similar data were obtained for Bcl-2-overexpressing metastatic B16 melanoma cells treated with SOD2 and $\mathrm{Bcl} 2$ antisense oligonucleotides. The treatment significantly reduced the number and viability of the cells and increased apoptosis, necrosis, and sensitivity to chemotherapy in the presence of tumor necrosis factor alpha $(\mathrm{TNF} \alpha)(22)$.

Studies investigating the role of SOD2 in radiation protection following irradiation cancer therapy in human neuroblastoma, Ewing sarcoma, breast cancer, bladder cancer, colon cancer, prostate cancer, and lung cancer cells demonstrated that low-dose irradiation (2-100 cGY) increased $\mathrm{NF} \kappa \mathrm{B}$ activation and consequently increased SOD2 expression, as well as the survival/clonal expansion of cells (11).
Hence, based on these data, SOD2 may represent a potential target for combination cancer therapy treatments.

\section{A. SOD2 in tumorigenesis}

Early reports concerning SOD2 function in carcinogenesis showed a correlation between decreased SOD2 expression at the early initial stage of carcinogenesis and suggested a tumor suppressor role for the enzyme $(59,262,289,397)$. However, SOD2 expression has been shown to increase in late-stage aggressive and metastatic cancers and cell models $(61,125,142,161,165$, $175,378,384)$, indicating the importance of the enzyme for the progression of cancer. The conclusion is corroborated by the data demonstrating SOD2-driven inhibition of apoptosis and increased mRNA expression of MMPs $M M P 1, M M P 2$, and $M M P 9$ in several cancers and cancer cell lines, such as HT1080 fibrosarcoma cells, MCF-7 breast cancer cells, 253J bladder carcinoma cells, and 253J-BV metastatic bladder cancer cells $(61,129,258,395)$ (Fig. 6A).

Decreased apoptosis correlating with increased SOD2 expression could be mediated by the stabilization of the mitochondrial membrane, decreased caspase 3 activation, and inhibition of TNF-related apoptosis-inducing ligand (TRAIL) (244). Increased SOD2-derived MMP expression was demonstrated to correlate with increased migration, increased invasion, and increased metastasis (Fig. 6A). The exact mechanism underlying how SOD2 increases $M M P$ gene expression and the metastatic potential of the cells has not been completely characterized, but it could be related to the modification of $M M P$ gene promoter region activity, manipulation of phosphatase and tensin homologue (PTEN), protein tyrosine phosphatase, nonreceptor type 12 (PTPN12), and MAP kinase phosphatase activity, and the maintenance of mitochondrial integrity and metabolic capacity $(125,126)$.

In addition, increased SOD2 expression is correlated with an increased epithelial-mesenchymal transition (EMT) score in different breast cancer subtypes, whereas the RNAi silencing of SOD2 decreases EMT-related protein (VIMENTIN, $\beta$ CATENIN, SLUG, N-CADHERIN, TWIST, zinc finger E-box binding homeobox 2 [ZEB2], and SNAIL) expression $(126,165,175,207)$, strongly suggesting a stimulatory role for SOD2 in cancer cell migration and metastasis.

The enhancer function of SOD2 in cellular migration and metastasis is further supported by data demonstrating the correlation of SOD2 expression and phosphorylation of breast cancer anti-estrogen resistance protein 1 (BCAR1), also known as p130Cas. The overexpression of SOD2 increases BCAR1 phosphorylation and membrane recruitment, evidently causing the oxidation inactivation of PTEN $(62,127)$. PTEN is a tumor suppressor gene that converts PIP3 to PIP2, counteracting the conversion of PIP2 to PIP3 by PI3K. Decreased PTEN activation allows the accumulation of PIP3, thereby maintaining downstream AKT kinase in the active state, which plays a fundamental role in the regulation of growth, proliferation, apoptosis, angiogenesis, and metabolic activities of the cells.

BCAR1 is a docking protein that has several proteinprotein interaction domains with a prominent role in cell adhesion and initiation of cell migration, creating the link between increased SOD2 expression and cellular cytoskeletal modification. The amino terminal SH3 domain of BCAR1 has been shown to interact with FAK and protein tyrosine kinase $2 \beta$ (PTK2 $\beta$ ), also known as the PYK2 nonreceptor 
tyrosine kinase of the FAK family, inducing their activation. The BCAR1 and PTK $2 \beta$ interaction with FAK induces adherent junction disassembly, modifications of focal adhesions, and cellular cytoskeletal structure. More importantly, FAK-BCAR1 signaling activates the small GTPase RAC1 and CDC42 (a member of the RHO small GTPase family) with the subsequent induction of membrane protrusions and cell migration $(63,127)$.

Recently, it was demonstrated that PKC-ERK1/2 signal transduction upregulates mitochondrial SOD2 expression in

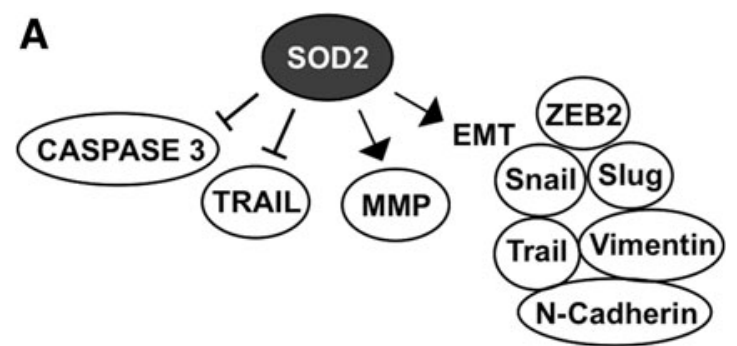

B

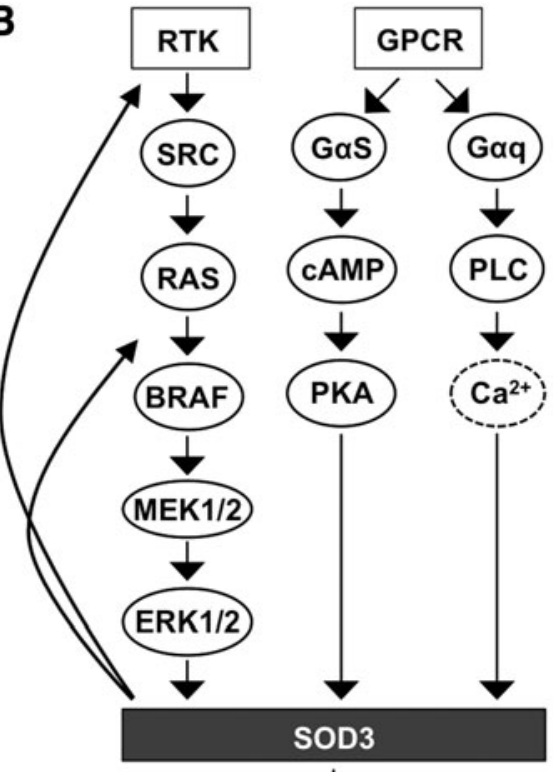
Survival

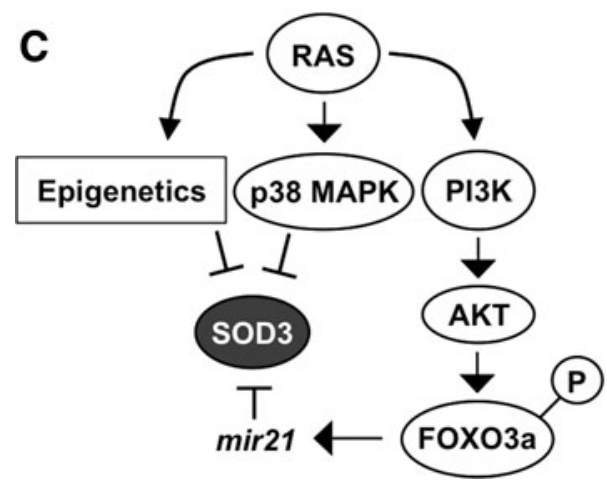

response to glucose starvation in lung carcinoma cells (170). Indeed, increased SOD2 expression parallels with glycolytic metabolism, especially in aggressive metastatic cancers, causing the activation of $5^{\prime}$ adenosine monophosphateactivated kinase (AMPK), which further stimulates glycolysis. In fact, a recent study demonstrated SOD2-driven increased AMPK phosphorylation at Thr172 in breast, colon, and prostate cancers, suggesting the involvement of SOD2 in the glycolytic switch in cancer progression that may represent a mechanism to regulate the Warburg effect. Interestingly, the data demonstrate the interaction of SOD2-derived cell metabolism via AMPK signaling and NADPH oxidase-derived ROS production.

In addition to modifications of glycolytic metabolism, SOD2 signal transduction via AMPK inhibits apoptosis and increases drug resistance and colony formation in vitro in soft agar, all characteristics of late-stage cancers (121). The in vitro results are further corroborated by clinical data, demonstrating the correlation between increased expression, poor survival, disease relapse, and metastasis in head and neck squamous cell carcinoma, pancreatic cancer, gastric cancer, and colorectal carcinoma $(84,165,288)$.

\section{Extracellular SOD, EC-SOD, SOD3}

Secreted extracellular SOD is the latest member of the mammalian SOD isoform family. The enzyme is composed of two covalently associated dimers, which form a tetrameric protein except for rat SOD3, which has a dimeric structure (38, 379). Each SOD3 subunit has four domains: (i) secretion domain; (ii) amino terminal glycosylated (Asn-89) domain, which increases the solubility of the enzyme; (iii) copper- and zinccontaining active center domain; and (iv) C-terminal heparan sulfate binding domain that contains a proteolysis-sensitive segment causing the sequential degradation of the enzyme with consequent loss of binding capacity to cell membranes.

Based on the cell membrane heparan sulfate affinity, SOD3 can be classified into three (A, B, and C) or five (I-V) subgroups: the intact secreted $\mathrm{C}$-form has all four C-terminal ends intact and is referred to as classes IV and V; the B-form has reduced affinity due to protease degradation and is referred to as classes II and III; and the A-form is soluble lacking C-terminal heparan sulfate binding domains and is referred to as class $I(4,162)$. The recent observations suggest

FIG. 6. SOD2 and SOD3 in cell metastasis and in cell survival. (A) SOD2 promotes metastatic signaling molecule activation and inhibits apoptosis by downregulating caspase 3 and TRAIL, and by upregulating EMT mediating proteins and MMPs. (B) RTK-SRC-RAS-ERK1/2, GPCR-cAMP-PKA, and GPCR-PLC-Ca ${ }^{2+}$ signaling activate SOD3 production, thereby increasing cell proliferation and survival. Positive feedback signaling increases RTK phosphorylation and RAS GTP loading, thus maintaining activity of the RASERK1/2 cascade. (C) RAS controls SOD3 expression through epigenetics, by p38MAPK activation, and by the PI3K-AKTFOXO3a pathway. PI3K-AKT activation causes phosphorylation of the transcription factor, FOXO3a, resulting in its transfer from the nucleus to the cytoplasm, thereby increasing mir21 synthesis, which targets SOD3 mRNA. EMT, epithelial/ mesenchymal transition; FOXO, forkhead box family; PI3K, phosphatidylinositol-4,5-bisphosphate 3-kinase; TRAIL, TNFrelated apoptosis-inducing ligand. 
that the functional response of the cell membrane-bound intact C-form SOD3 on cells and tissues is mediated in an autocrine and a paracrine manner (2, 192, 195, 275). A number of reports have demonstrated that SOD3 has antioxidative, antiapoptotic, anti-inflammatory, and growthpromoting characteristics in tissue injury models, in genetically modified mice, and in cancer models $(39,91,194,195$, 197, 223). Indeed, SOD3 was first identified as a therapeutic enzyme being able to inhibit efficiently liver damage in a paracetamol intoxication model, cardiovascular damage in a reperfusion model, and neointima growth in a restenosis model and only recently shown to promote unwanted growth in cancer models $(39,184,189,190,192,194,195,197,332)$.

The signal transduction pathways RAS-BRAF-MEK1/2ERK1/2, GPCR-G $\alpha$ s-cAMP-PKA, and GPCR-G $\alpha$ q-PLC$\mathrm{Ca}^{2+}$, which increase the enzyme levels $(34,93,184,197)$, and p38MAPK, which inhibits the expression (3), are the most prominent signaling cascades regulating SOD3 synthesis. Interestingly, increased SOD3 expression has a stimulatory effect on RAS activation by GTP loading, suggesting a positive feedback loop: SOD3 increases RAS activation, which induces downstream signaling, causing increased SOD3 production (Fig. 6C) $(190,197)$. More interestingly, the positive feedback loop, which maintains the mitogenic RAS-ERK1/2 signal pathway active, could potentially represent a mechanism underlying how SOD3 mediates tissue injury healing.

RAS is fundamentally connected to SOD3 signaling by regulating SOD3 mRNA synthesis through the MEK1/2-ERK1/ 2 cascade and by inhibiting gene expression through p38 MAPK phosphorylation, methylation, and acetylation. In addition, RAS activates the PI3K-AKT pathway, which induces FOXO3a inactivation by phosphorylation, with consequent increased mir 21 synthesis, and targets SOD3 mRNA, interfering gene expression $(33,185,396)$ (Fig. 6C). Although SOD3 expression at physiological levels supports growth, the enzyme is paradoxically downregulated in certain cancers. The gradual inhibition of SOD3 expression correlates with the oncogene activation level involving small GTPase regulatory genes, epigenetic regulation of gene expression, mir21 stimulation by AKT-FOXO3a phosphorylation, and p38MAPK signaling (33, 184, 190, 262, 393, 394, 396) (Fig. 6C).

The fine-tuning of RAS downstream signaling is controlled by RAS GTPase regulatory genes guanine nucleotide exchange factor (GEF), GAP, and guanine nucleotide disassociation inhibitor (GDI), which have a balancing effect on SOD3 expression levels. Moderately 6- to 10-fold increased RAS activation stimulates SOD3 mRNA synthesis until there is a sudden decrease in the enzyme mRNA production at more aberrant RAS activation levels. Simultaneously with decreased SOD3 mRNA expression, there is an inverse correlation with increased mir21 expression and increased p38 MAPK phosphorylation, both induced by RAS (33). The microRNA mir21 has been shown to bind to $3^{\prime}$ untranslated region (UTR) in SOD3 mRNA, thus causing the degradation of the messenger (396). In advanced cancers, RAS-induced epigenetic methylation and acetylation have been shown to contribute to more pronounce silencing of SOD3. Therefore, the regulation of SOD3 expression can be divided into reversible regulation by small GTPase regulatory genes, mir21, or p38MAPK and irreversible epigenetic silencing by methylation and acetylation (33).

\section{A. SOD3 in tumorigenesis}

Similar to SOD2, early studies have suggested that SOD3 is a tumor suppressor gene downregulated in cancer. Various works using transgenic SOD3 mice or adenovirus gene transfer techniques have demonstrated the inhibitory function of SOD3 on cancer cell proliferation and tumor growth. In all these early studies, the expression level of the enzyme was highly above the physiological levels observed in tissues and cells $(346,347,377)$ causing aberrant growth signal affecting small GTPases and $\beta$-CATENIN signaling (190). More recent works have demonstrated a dose-dependent growth response for the enzyme, clarifying the controversy between recently and previously published data and demonstrating that, at the physiological level or at moderately increased level, SOD3 supports cell proliferation and cell survival, and reduces apoptosis by regulating RAS activation $(39,189,190,197,368)$.

Importantly, increased SOD3 expression results in increased phosphorylation of various growth-related cell membrane RTKs and RTK-associated signaling molecules, such as SRC proto-oncogene family members, regardless of the SOD3 expression level. It is not quite clear how enzyme overexpression increases RTK phosphorylation, but it could be mediated by the ability of $\mathrm{H}_{2} \mathrm{O}_{2}$ to oxidatively inactivate PTPs by modifying the catalytic site cysteine amino acids, thereby causing persistent RTK activation as a response of ligand binding to the receptor $(266,267,354)$. Therefore, SOD3 itself would not be a growth factor, but the enzyme, by producing $\mathrm{H}_{2} \mathrm{O}_{2}$, could represent a regulator of growthrelated signaling molecule activity.

The regulatory role of SOD3 in signaling is further corroborated by data suggesting SOD3 dose-dependent activation of small GTPases. At high SOD3 levels, the expression of RAS regulatory gene $G E F$ mRNA synthesis is downregulated, whereas $G A P$ s and $G D I$ s, which maintain RAS in the inactive GDP-bound form in the cytosolic compartment, are increased. Thus, SOD3-driven modification of small GTPase regulatory gene expression inhibits the progression of signal transduction downstream of RAS. Moderately increased ( $\sim 2$ - to 4-fold increased enzyme activation level and 10- to 15-fold increased mRNA level) SOD3 expression stimulates GTP loading to small GTPases, thus allowing cell membrane signal transduction to pass RAS to the downstream signaling network $(190,197)$ (Fig. 7A, B).

Other control checkpoints for signaling observed at high SOD3 expression levels include, for example, $\beta$-CATENIN entry into the nucleus. High SOD3 concentration increases the expression of WWTRl (WW domain containing transcription regulator 1) and AXIN2 genes, which maintain $\beta$ CATENIN in the cytoplasm. The growth regulatory role of SOD3 is further strengthened by KEGG and GO functional pathway analysis, suggesting the highest impact on growth and proliferation signaling $(190,197)$ (Fig. 7C).

Although the expression of the enzyme is gradually downregulated in thyroid cancers and thyroid cancer cell lines, a recent article demonstrated increased SOD3 mRNA synthesis in PTC MSCs, therefore suggesting an autocrine/ paracrine switch in SOD3 production (39, 184, 275). Increased SOD3 expression in tumor stroma MSCs stimulated thyroid cancer cell proliferation but decreased cancer cell migration, indicating that SOD3 may reduce the intratumoral affinity of cancer cells and allows them to migrate locally 


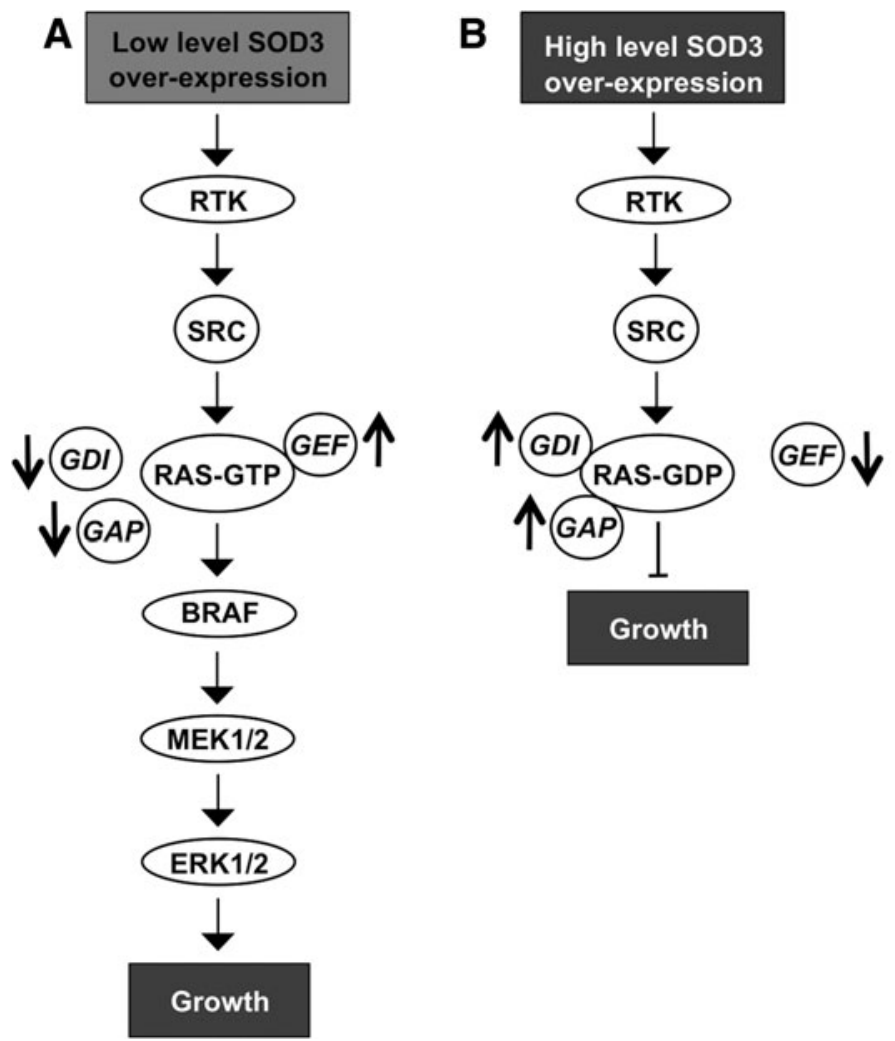

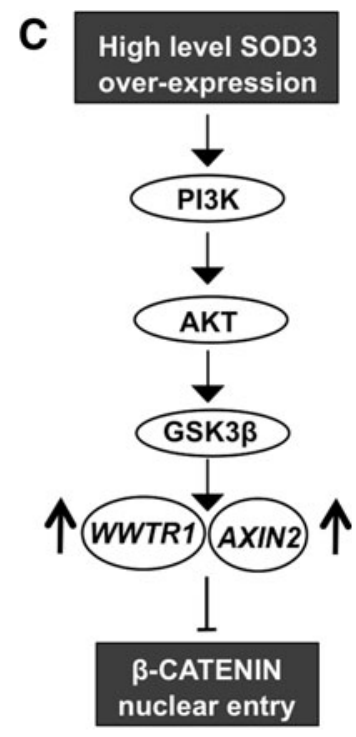

FIG. 7. SOD3 regulation of signal transduction. (A) Low level of SOD3 expression increases RTK and SRC phosphorylation and allows signaling through small GTPase RAS by increasing GEF expression and decreasing GAP and GDI expression. (B) High level of SOD3 expression increases RTK and SRC phosphorylation but inactivates small GPTase RAS by decreasing GEF expression and increasing GAP and GDI expression. Thereby, the signal does not proceed to mitogenic pathway. (C) High level of SOD3 expression increases WWTR1 and AXIN2 expression inhibiting $\beta$-CATENIN nuclear entry, which then attenuates growth. AXIN2, axis inhibition protein 2; GDI, guanine nucleotide disassociation inhibitor; GEF, guanine nucleotide exchange factor; WWTR1, WW domain containing transcription regulator 1. toward peritumoral regions (275). Previously, SOD3 has been shown to reduce cellular migration by downregulating inflammatory cytokines and intracellular adhesion molecule 1 (ICAM-1), vascular cell adhesion molecule 1 (VCAM-1), and E-selectin and P-selectin adhesion (198) molecules, which are involved in cell/cell interaction structures, such as in tight junctions. Therefore, the data may suggest that, at the late phase of carcinogenesis, the autocrine/paracrine switch maintains SOD3-driven growth support but, at the same time, releases epithelial cancer cells to migrate toward normal healthy tissue (275).

\section{Interaction of NOX1-5- and SOD1-3-Associated Signaling}

\section{A. GPCR signaling}

GPCRs could be the most important signaling elements that integrate NOX1-5 and SOD1-3 signal transduction. GPCRs form the largest family of cell membrane-associated signaling molecules consisting of over 800 members expressed in all cell and tissue types. GPCR is activated by many ligands representing peptides and proteins, lipids, amino acids and ions, biogenic amines, and a heterogenic group of substances, such as odorants, nucleotides, endorphins, and even light. GPCRs are integral cell membrane receptors that contain seven transmembrane $\alpha$-helical regions and bind to a wide range of extracellular ligands. Ligand binding to GPCR initiates a conformational change in the receptor structure, allowing interaction with the heterotrimeric large $G$ protein subunit $\alpha\left(\alpha_{\mathrm{s}}\right.$, $\alpha_{\mathrm{i}}, \alpha_{\mathrm{q}}$, or $\alpha_{12}$ ) with consequent GDP to GTP catalysis activating the large $\mathrm{G}$ protein. On activation, the $\mathrm{G}$ protein subunit $\beta\left(\beta_{1-5}\right)$ and subunit $\gamma\left(\gamma_{1-14}\right)$ dissociate from $\mathrm{G} \alpha$ and function as a heterodimer stimulating $\mathrm{PI} 3 \mathrm{~K}, \mathrm{PLC} \beta$, and, in some cases, also small GTPase RAS and RHO activation. Large $\mathrm{G}$ protein signaling activation continues until GTP is hydrolyzed from the $\mathrm{G} \alpha$ subunit, which then adheres back to the $\mathrm{G} \beta \gamma$ dimer, thereby ending the downstream pathway activation $(81,221$, 261) (Fig. 8A).

Characteristically, large $\mathrm{G} \alpha$ proteins are divided into different groups according to the large $G$ protein they interact with: (i) $\mathrm{G} \alpha_{\mathrm{i}}$ (adenylyl cyclase inhibitor) inhibits cAMP synthesis but activates the SRC proto-oncogene family and downstream MAPK growth, angiogenesis, and survival signaling; (ii) $\mathrm{G} \alpha_{\mathrm{s}}$ subunit antagonizes $\mathrm{G} \alpha_{\mathrm{i}}$ function-activating adenylyl cyclase and cAMP-dependent PKA activation that phosphorylates CREB transcription factor with the consequent increased expression of target genes involved in DNA repair, oncogenesis, and autophagy; (iii) $\mathrm{G} \alpha_{\mathrm{q}}$ proteins activate PLC signaling that catalyzes the formation of IP3 and $\mathrm{DAG}$, leading to the activation of PKC; and (iv) $\mathrm{G} \alpha_{12}$ downstream target molecule small GTPase RHO and RAC modify actin cytoskeleton reorganization and focal adhesions and are frequently involved in carcinogenesis (283).

GPCRs regulate essentially almost all normal cellular functions, such as blood pressure, by altering the heart rate, vascular resistance, and fluid/electrolyte balance. Regulation of the immune system activation by controlling immune cell chemotaxis, homing, activation, and target tissue recruitment is another important physiological response of GPCRs, as well as kidney sodium secretion, and bone mass and architecture modeling. Because $\sim 90 \%$ of GPCRs are expressed in the brain, their physiological function is foremost characterized in the neuronal environment (292). According to recent studies, transforming cells utilize the normal 
physiological functions of GPCRs to promote their autonomous proliferation, escape from immune recognition, and survive during metastatic invasion, intravasation, and extravasation $(81,221,261)$.

GPCRs have been shown to mediate NOX4-derived ROS production in heart failure on ligand binding (Fig. 8A).
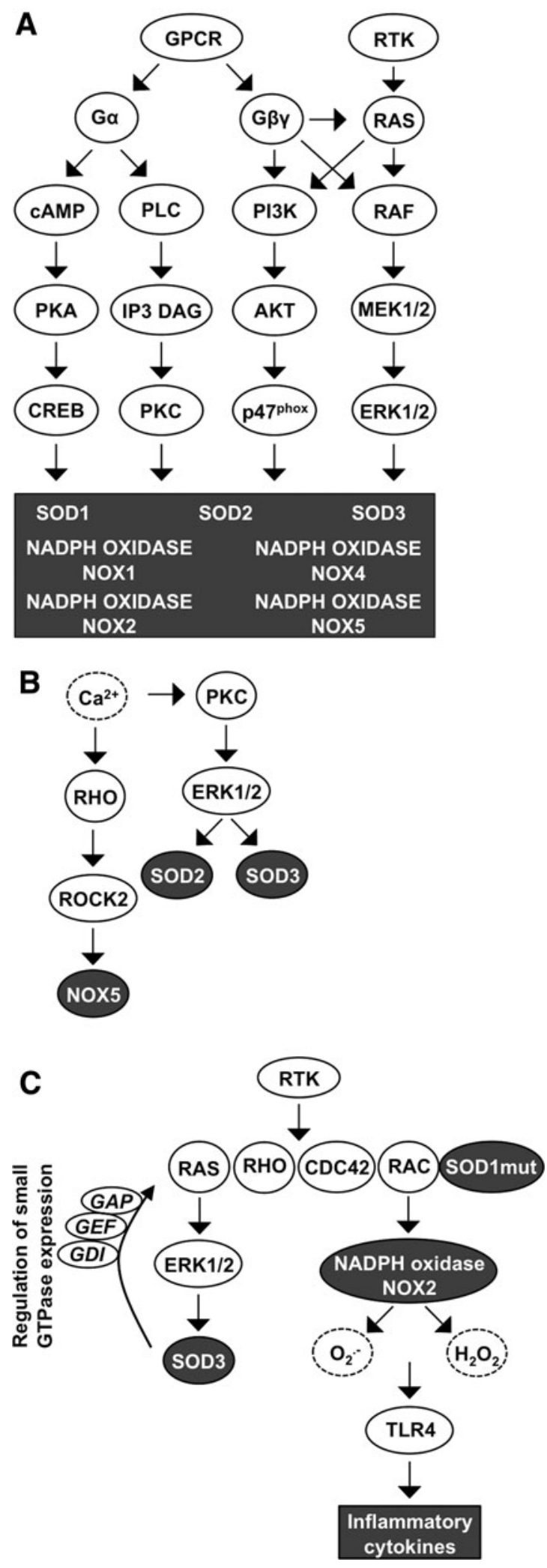

Binding of the agonist to the receptor induces the phosphorylation of G-protein-coupled receptor kinase 2 (GRK2) and recruitment of $\beta$-arrestin, initiating downstream signaling and increasing NOX4 activity. Increased ROS production by NOX4 augments myocardial dysfunction and DNA damage, and initiates apoptotic cell death program in cardiac myocytes, thereby increasing the risk for chronic heart failure (348). In astrocytes, ATP ligand binding to purinoceptor 7 (P2X7) receptors, which represent P1 GPCRs, has been demonstrated to augment NOX4 expression and activation. Altered ROS production in microglia has a direct impact on the pro/anti-inflammatory role that cells may play in neurodegenerative diseases, such as Alzheimer's disease, Parkinson's disease, and in ALS (232).

Nucleotides, such as ATP, activate PY2 purinergic GPCRs, inducing PLC-cAMP-Ca ${ }^{2+}$ signal transduction, protecting astrocytes against ROS-derived damage. The interaction between GPCR and SODs is highlighted by the data suggesting increased SOD2 and SOD3 expression caused by the activation of purinergic P2Y GPCR and thyroidstimulating hormone receptor (TSHR) class A GPCR. Mechanistically, ligand binding to GPCR class A and purinergic P2Y GPCR activates the $\mathrm{G} \alpha_{\mathrm{s}}$-cAMP-PKA pathway and/or $\mathrm{G} \alpha_{\mathrm{q}}$ PLC-Ca ${ }^{2+}$ signaling, increasing the expression of SOD2 and $S O D 3$, which then regulate normal thyrocyte proliferation and resistance against apoptosis $(93,184)$ (Fig. 8A).

Another unstudied connection between SOD3- and GPCRmediated growth signaling comes from data demonstrating the regulation of regulator of $\mathrm{G}$ protein signaling 4 (RGS4) expression by SOD3 (190). The results observed in metastatic anaplastic thyroid cancer cells suggest a putative role for the enzyme in GPCR signaling, although currently there are no reports showing SOD3-driven regulation of large $\mathrm{G}$ protein signaling. SOD1 activation has been studied in astrocytes in which peptides, such as octadecaneuropeptide (ODN), can bind $\mathrm{G}_{1 / 0}$ GPCR, resulting in markedly increased SOD1 expression already minutes after ligand binding. The signaling increasing SOD1 production is specifically channeled via PKA as its inhibitor, $\mathrm{H} 89$, has been reported to abrogate enzyme production (117) (Fig. 8A).

GPCR signaling activating small GTPase RAC is frequently associated with actin modification. Large $\mathrm{G} \alpha_{12}$ activation initiates cytoskeletal remodeling affecting focal adhesion integrity, thereby allowing cells to migrate locally $(81,221,261)$. Similar to $\mathrm{G} \alpha_{12}$, noncanonical WNT

FIG. 8. Interaction of GPCR, $\mathrm{Ca}^{2+}, \mathrm{RTK}$, and small GTPase signaling affecting redox gene expression. (A) GPCR $\mathrm{G} \alpha$ and $\mathrm{G} \beta \gamma$ activation increases signaling through cAMP, PLC, small GTPases, PI3K-AKT, RAS, and RAF, which are among the redox-linked signal transduction molecules, thereby stimulating expression of SOD1-3 and NADPH oxidases NOX1, $-2,-4$, and -5 . RTK activation occurs at the level of RAF and PI3K signaling molecules. (B) $\mathrm{Ca}^{2+}$ increases small GTPase RHO-ROCK signaling that stimulates $N O X 5$ expression. $\mathrm{Ca}^{2+}$ activates also PKCERK1/2 pathway causing increased SOD2 and SOD3 production. (C) RTK and small GTPase activation stimulate ERK1/2 signal transduction that promotes SOD3 expression and formation of a regulatory loop affecting small GTPase activation. Mutant SOD1 binds to small GPTase RAC in a redox-insensitive manner causing continuous ROS production and inflammatory cytokine synthesis. 
(wingless-type MMTV integration site family) signaling also recruits RAC, as well as RHO and CDC42, inducing cytoskeletal reorganization. RHO, which signals through ROCK, regulates actin filament assembly to form mainly lamellipodia, whereas RAC and CDC42 promote actin polymerization at the cell periphery, constructing primarily filopodia (150, 228). Under certain conditions, GPCR and noncanonical WNT signaling stimulate the PI3K-AKT pathway, which are, as explained above, involved in $\mathrm{p} 47^{\text {phox }}$ phosphorylation, initiating NAPDH NOX assembly $(40,137,180)$. However, the activation of PI3K-AKT signaling is more thoroughly studied in the context of RTK phosphorylation that induces SRC proto-oncogene activation in addition to downstream ERK1/2 and p38MAPK signal transduction, all connected to redox signaling (Fig. 8A).

Chemokine receptors represent a class of GPCRs composed of $\sim 20$ members that mediate various cellular events, such as cell migration and survival. CXCR4/SDF-1 interaction has been shown to mediate the homing and quiescence of hematopoietic stem cells (HSCs); however, in cancer, CXCR4positive cells are more prone to metastasis (187). In prostate cancer cells, SOD1 has been reported to make a direct contact with the first intracellular loop of CXCR4 in an SDF-1 ligand binding-dependent manner. Interaction of SOD1 with CXCR4 phosphorylates AKT, inhibiting the function of proapoptotic proteins, such as Bcl-2-associated death promoter (BAD), and activating antiapoptotic signal transduction (392).

\section{B. $\mathrm{Ca}^{2+}$ signaling}

The most noticeable factor connecting redox enzymes to cellular functions is the reciprocal signaling of ROS and $\mathrm{Ca}^{2+}$, which coordinates cellular functions in cancer, such as metabolism and gene expression, cell fate, apoptosis or growth, in a dose-dependent manner. The biological response of $\mathrm{Ca}^{2+}$ is caused by direct binding to target proteins without catabolic or anabolic modifications (112). Interestingly, cell adhesion to the vascular wall is enhanced by mitochondrial ROS production stimulated by GPCR-Ca ${ }^{2+}$ signaling.

Mechanistically, ligand binding to GPCR stimulates the interaction of IP3 to its mitochondrial membrane receptor InsP3R, increasing the mitochondrial matrix $\mathrm{Ca}^{2+}$ concentration. The released $\mathrm{Ca}^{2+}$ then stimulates mitochondrial NADH and $\mathrm{O}_{2}{ }^{\bullet-}$ production as a part of ATP generation (123). In support of this, recent data demonstrate that increased $\mathrm{O}_{2}^{\bullet-}$ promotes $\mathrm{NF} \kappa \mathrm{B}$ signaling, which stimulates ICAM-1 expression, which is critical for leukocyte adhesion to vascular endothelial cells. In addition, ICAM-1 expression is inhibited by the SOD mimetic MnTBAP (123) and by increased expression of SOD3 (198), corroborating conclusions that cell adhesion molecule expression could be $\mathrm{O}_{2}^{\bullet-}$ dependent.

As mentioned above, $\mathrm{Ca}^{2+}$ functions as a mediator of GPCR signal transduction, influencing the redox enzyme expression level and mediating the ROS-derived message into the cellular signal transduction network. ROS, especially $\mathrm{H}_{2} \mathrm{O}_{2}$, directly control the intracellular $\mathrm{Ca}^{2+}$ concentration by oxidizing cysteine residues within $\mathrm{Ca}^{2+}$ channels or within $\mathrm{Ca}^{2+}$ channel activators, such as stroma interacting molecule1 (STIM1), affecting protein conformation and activity (128, 199). Similar to mitochondrial $\mathrm{Ca}^{2+}$ influx, cell membrane GPCR-derived IP3 can interact with IP3R at the endoplasmic reticulum, which is one of the major intracellular $\mathrm{Ca}^{2+}$ stor- age sites in addition to sarcolemma, allowing $\mathrm{Ca}^{2+}$ flow into the cytoplasm (78). Modifications in the intracellular $\mathrm{Ca}^{2+}$ concentrations consecutively activate downstream signaling, stimulating the PKC pathway that causes increased NADPH NOX and SOD activation (128) (Fig. 8B).

Although $\mathrm{Ca}^{2+}$ does not directly activate NOX1 and NOX3, it is involved in the stimulation of upstream small GTPase, thereby increasing signal transduction and leading to NADPH NOX1 and NADPH NOX3 complex activation $(19,29)$. GPCR signal transduction inducing the IP3 pathway and increased intracellular $\mathrm{Ca}^{2+}$ concentration have been shown to activate the small GTPase RHO-ROCK2 cascade that sequentially increases NOX5 mRNA production (29). NAPDH NOX5-derived $\mathrm{O}_{2}^{\bullet-}$ production is directly dependent on $\mathrm{Ca}^{2+}$, which induces a conformational change connecting the $\mathrm{N}$-terminal regulatory site with the C-terminal catalytic region. The change in the three-dimensional EF motif structure could be caused by the reorganization of Cys109 residues, allowing the $\mathrm{N}$-terminal EF motif to contact the catalytic $\mathrm{C}$-terminus that contains binding sites for FAD and NADPH (16) (Fig. 8B).

In ALS models, $\mathrm{Ca}^{2+}$ has been demonstrated to cause conformational changes, increasing SOD1 $\beta$-sheet structures and consequently increasing neurotoxic SOD1 aggregation into the cells, therefore promoting disease progression (200). SOD2 is involved indirectly in increased $\mathrm{Ca}^{2+}$ accumulation by inhibiting PTEN activation. $\mathrm{H}_{2} \mathrm{O}_{2}$ produced by SOD2 induces oxidative inactivation of PTEN by conversion of the sulfhydrul groups to a disulfide causing more compact protein structure. The PTEN tumor suppressor inhibits PI3Kderived PIP2 catalysis to PIP3. SOD2 function then increases the cellular content of PIP2, which also functions as a mediator of GPCR signaling, stimulating the PLC-IP3/DAG signaling pathway and leading to increased $\mathrm{Ca}^{2+}$ synthesis.

Interestingly, increased $\mathrm{Ca}^{2+}$ has been shown to activate the PKC-ERK1/2 cascade, which increases SOD2 expression, therefore suggesting a feedback loop in the regulation of SOD2 activation $(62,127,128)$ (Fig. 8B). $\mathrm{Ca}^{2+}$ signaling was shown to regulate SOD3 expression as the GPCR-PLC-Ca ${ }^{2+}$ signaling pathway increases SOD3 mRNA expression to stimulate thyroid cancer cell proliferation, as mentioned above (184) (Fig. 8B). The reciprocal action of ROS and $\mathrm{Ca}^{2+}$ is not limited to redox enzyme activation but plays a prominent role in cellular energy production by promoting ATP synthesis, the Krebs cycle, and oxidative phosphorylation in mitochondria. $\mathrm{Ca}^{2+}$ is even needed for neutrophil activation and in phagosome formation, suggesting a role in the host defense against invading microbes (112) and demonstrating the ample responsibility in the regulation of cellular functions.

\section{RTK and small GTPase signaling}

The RTK family contains 58 members divided into 20 classes mediating the signaling response of growth factors, cytokines, and hormones into the intracellular signaling network. Ligand binding to the receptor induces structural changes, stimulating the tyrosine kinase phosphorylation activity of downstream signaling molecules or neighboring RTK (202). SOD3 has been shown to stimulate RTK phosphorylation by the increased production of $\mathrm{H}_{2} \mathrm{O}_{2}$ (190). The mechanism underlying SOD3-derived RTK phosphorylation in the absence of supplemental ligands may be related to the 
ability of $\mathrm{H}_{2} \mathrm{O}_{2}$ to affect PTPs, thereby allowing RTK activation (266). This is supported by data suggesting that SOD3 overexpression nonspecifically increases the phosphorylation of RTKs stimulating subsequent membrane-associated SRC family phosphorylation and small GTPases RAS, RAC, CDC42, and RHO (190, 197) (Fig. 8C).

The small GTPases are monomeric proteins that appear active when associated with GTP and inactive when they hydrolyze GTP to GDP. The activity of small GTPases is controlled by small GTPase regulatory proteins GEFs, GAPs, and GDIs. GEF stimulates GTP loading to small GTPase, thus activating it, whereas GAP catalyzes the hydrolysis of GTP to GDP, and GDI inhibits GDP dissociation from small GTPase and maintains small GTPase in the cytosolic compartment. Once GDI and GAP expression is downregulated, small GTPase associates in the GTP bound active form at the cell membrane by the action of GEF $(26,73)$. Interestingly, small GTPase activity is affected also by SOD1, especially by mutant SOD1, by direct interaction, as demonstrated in ALS models.

As described above, the interaction between small GTPase RAC1 and SOD1 is enhanced by mutations in the SOD1 gene that maintains downstream RAC1 signaling, leading to increased inflammatory cytokine production and the risk of ALS development $(120,157)$ (Fig. 8C). In addition to being involved in cytokine and ROS production, as part of the NADPH oxidase complex, RAC is engaged in cell cycle progression, transformation, induction of actin polymerization, regulation of membrane ruffling, and adhesion of cells to the ECM or neighboring cells, thereby regulating movement, tissue morphogenesis, cancer initiation, and metastasis $(229,361)$.

\section{Oncogene signaling}

The activation of individual oncogenes mediates redox signal transduction to downstream target molecules (Table 1). The SRC proto-oncogene has been demonstrated to activate NADPH oxidases NOX1-, NOX3-, NOX4-, and NOX5induced $\mathrm{O}_{2}{ }^{-1}$ production in cancer models $(87,105,107,171)$ and to increase mutant SOD1 aggregate formation in ALS. Remarkably, SRC signal transduction can selectively activate only NOX1 and NOX3 without activating NOX2 or NOX4 in cases where the signaling is mediated by downstream TKS4 and TKS5 (107).

In primary cell transformation, SRC interacts with small GTPases by inducing persistent activation of RAC1 through tyrosine phosphorylation of RAC1 GEF VAV2 (105, 171, 173, 323). Furthermore, RAC1 has a crucial role in RAS oncogene-induced ROS production and activates oncogeneinduced proliferative bursts, growth arrest, and escape from premature senescence, consequently causing immortalization and transformation of primary cells $(263,321,322)$.

EGFR-SRC signaling-stimulated NADPH oxidase NOX4 enhances anoikis (cell detachment-induced apoptosis) resistance and survival of cancer cells, which are detached from the ECM but are still anchorage dependent and thereby sensitive to anoikis. Characteristically, cells that are released from stromal components lose the survival signaling provided by integrins that cause cell death initiation. However, cancer cells may gain resistance to anoikis, enabling the cells to intravasate, survive in vasculature, and extravasate into distant tissue sites $(341,344)$.
Table 1. Growth Promoters Affecting Reduction/Oxidation Gene Activation

\begin{tabular}{|c|c|c|}
\hline $\begin{array}{l}\text { Growth } \\
\text { promoter }\end{array}$ & $\begin{array}{l}\text { Associated } \\
\text { redox gene }\end{array}$ & References \\
\hline BRAF & SOD3 & $(33,196)$ \\
\hline cABL & NOX5 & (86) \\
\hline E1A & NOX4 & (34) \\
\hline EGF & SOD1 & $(157)$ \\
\hline EGFR & NOX3, NOX4 & $(170,172,203)$ \\
\hline FLT-ITD & NOX4 & $(154,155)$ \\
\hline IGF & SOD1 & $(157)$ \\
\hline MCF7 & SOD1 & (272) \\
\hline MCF-10A & SOD1 & (272) \\
\hline MDA-MB157 & SOD1 & $(272)$ \\
\hline MDA-MB-231 & SOD1 & (272) \\
\hline PDGF & NOX1, NOX5 & $(153,187)$ \\
\hline PI3K & $\begin{array}{l}\text { NADPH oxidase } \\
\text { complex } \\
\text { activation }\end{array}$ & (139) \\
\hline PKA & NOX1 & $(71,181,388)$ \\
\hline $\mathrm{PKC}$ & NOX1, NOX2 & $(107,332,388)$ \\
\hline RAS & $\begin{array}{l}\text { NOX1, NOX4, } \\
\text { SOD2, SOD3 }\end{array}$ & $\begin{array}{c}(5,34,39,188 \\
189,195,196,272)\end{array}$ \\
\hline RGS4 & SOD3 & (189) \\
\hline SRC family & NOX1-5, SOD3 & $\begin{array}{c}(45,56,104,106 \\
170,172,322)\end{array}$ \\
\hline
\end{tabular}

EGF, epidermal growth factor; FLT-ITD, FMS-like tyrosine kinase with internal tandem duplication; IGF, insulin growth factor; PDGF, platelet-derived growth factor; PI3K, phosphatidylinositol4,5-bisphosphate 3-kinase; PKA, protein kinase A; PKC, protein kinase C; redox, reduction/oxidation; RGS4, regulator of $\mathrm{G}$ protein signaling 4; SOD, superoxide dismutase.

Involvement of SRC in redox signaling has also been investigated in neuronal degeneration models. A recent study utilizing induced pluripotent stem cells (iPSCs) derived from ALS patient with SOD1 mutations suggested that increased phosphorylation of SRC-ABL (Abelson murine leukemia viral oncogene homologue 1) causes the degeneration of motor neurons differentiated from iPSCs. Interestingly, the inhibition of SRC-ABL inhibited motor neuron degeneration by reducing SOD1 misfolding and restoring energy homeostasis (144).

In oncogene-driven mammary tumor models, such as the MCF-10A, MCF7, MDA-MB-231, and MDA-MB157 cell lines and (MMTV)-ErbB2, MMTV-Myc, and MMTV-Wnt transgenic mice, SOD1 is overexpressed in $60-100 \%$ of cases (273), suggesting that SOD1 activation is not linked to a specific oncogene and does not solely depend on oncogene activation. In contrast, SOD2 is downregulated by oncogenes, such as RAS, in the early phases of carcinogenesis (273), whereas RAS activation initially increases SOD3 production at low oncogene activation levels with consequent downregulation of SOD3 at high oncogene activation levels (33).

\section{Altered ROS Levels Are Associated with Progression of Tumorigenesis}

In numerous cancers, aberrant ROS production regulates proliferation, survival, angiogenesis, and stroma remodeling. NADPH oxidase-derived sustained ROS production in the liver can contribute to chronic inflammation, which in certain 
cases may result in hepatocellular carcinoma development (89). ROS synthesis by NADPH oxidase NOX5 and SOD2 has been suggested to contribute to progression of gastroesophageal reflux disease, which characteristically exposes the esophageal tissue to acids causing increased intracellular $\mathrm{Ca}^{2+}$-ROCK2 signaling and upregulation of NOX5 mRNA synthesis, to esophageal adenocarcinoma (139). NADPH oxidase NOX5-S-derived ROS then promote cell proliferation and survival (94), whereas SOD2, stimulated by NF $\kappa$ B and ZEB2, is involved in EMT of esophageal adenocarcinoma cells (175). The association of SOD2 with aggressive metastatic cancer phenotype is further supported by positive correlation of increased SOD2 expression with consequent $\mathrm{H}_{2} \mathrm{O}_{2}$-related $M M P 9$ and VEGF mRNA synthesis in bladder cancer (129).

In melanoma, ultraviolet (UV) radiation is the main external factor increasing ROS production, although the mechanism of melanoma molecular pathogenesis is heterogeneous. Skin exposure to UV results in a dose-dependent response in $\mathrm{H}_{2} \mathrm{O}_{2}$ production in melanocytes that directly corresponds to increased DNA damages. Noteworthy, somatic mutations, such as BRAF V600E, and activation of PI3K-AKT signaling pathway further increase ROS production, thus driving the progression of melanoma carcinogenesis (365). Increased expression of NADPH oxidase NOX4 has been shown to regulate cellular cycling, anchorage-independent growth, and in vivo tumorigenicity, thereby suggesting that oxidase is required for malignant transformation and increased tumorigenesis (389).

Other cancers that associate with NADPH oxidase NOX4 include prostate cancer, pancreatic cancer, and urothelial carcinoma. NOX4 is highly expressed in prostate cancer cell lines and in prostate cancer tissues compared with normal prostate cell lines or benign prostate tissues, respectively (243). NADPH oxidase NOX4 may further induce prostate tumor stroma fibroblast differentiation to myofibroblasts via activation of JNK and its downstream transcription factors, thereby coordinating TGF $\beta$ response in stroma remodeling (312). In pancreatic cancer models, H-RASV12 oncogene induces ROS production in RAC1 and NADPH oxidase NOX4-dependent manner (263).

ROS then promote SRC-mediated phosphorylation of protein kinase D1 at Tyr95, Tyr432, Tyr436, and Tyr502 with consequent activation of $\mathrm{NF} \kappa \mathrm{B}$, increased expression of RTK EGFR, and its ligands EGF and TGF $\alpha$. NF $\kappa \mathrm{B}$ may also be involved in the activation of SOD2, thus increasing the mitochondrial ROS production causing mitochondrial dysfunction (80). In urothelial carcinoma, NOX4, which is detected already in precancerous and in early noninvasive urothelial carcinoma, has been shown to stimulate cell cycle progression and cancer cell growth (329). Therefore, the continuous reciprocal interaction of redox enzymes and cancer-related signal transduction pathways regulates the redox balance and cancer progression.

\section{ROS in Thyroid Cancer}

\section{A. Redox-related signaling in thyroid tumorigenesis}

Thyroid tumors are among the most thoroughly characterized neoplastic diseases consisting of both benign and malignant forms, hence forming a well-characterized platform for redox enzyme studies. In thyroid tissue, TSHR, representing GPCR, mediates downstream signaling via
cAMP-PKA and PLC-Ca ${ }^{2+}$ pathways, which stimulate redox enzyme expression. In the pathway leading to thyroid hormone $\mathrm{T}_{3}$ and $\mathrm{T}_{4}$ synthesis, GPCR subunits $\mathrm{G} \alpha \mathrm{q} / 11$ and $\mathrm{G} \beta \gamma$ stimulate PLC $\beta$, which recruits IP3 and DAG formation, eventually increasing $\mathrm{Ca}^{2+}$ mobilization and activating downstream signaling, leading to thyroid cell differentiation and thyroid hormone production (166). Aberrations in the availability of the thyroid-stimulating hormone (TSH) ligand or mutations in downstream cascades disrupt the delicate signaling network, resulting in various thyroid pathologies; thus, the constant stable level secretion of TSH is important for normal thyroid function. Decreased iodide uptake results in reduced $\mathrm{T}_{3}$ and $\mathrm{T}_{4}$ hormone synthesis (hypothyroidism), which is compensated by increased TSH production.

Increased TSH levels activate downstream growth signal transduction, causing thyroid enlargement known as goiter, the most common thyroid disorder characterized by hyperplasia and hypertrophy. Although goiter can be associated with autonomous adenoma, histologically and functionally, it differs from thyroid adenomas (380).

Thyroid tumors originate from follicular and parafollicular $\mathrm{C}$ cells by genetic and epigenetic alterations, with consequent uncontrolled activation of signaling pathways maintaining thyroid cancer progression. Until now, the observed mutated genes include $B R A F, R A S$, phosphatidylinositol-4,5bisphosphate 3-kinase catalytic subunit $\alpha(P I 3 K \alpha), P T E N$, TP53, $\beta$-CATENIN, anaplastic lymphoma kinase $(A L K)$, isocitrate dehydrogenase 1 (IDH1), rearrangement of rearranged during transfection (RET)-PTC, and paired box 8 (PAX8)peroxisome proliferator-activated receptor $\gamma(P P A R \gamma)$ resulting in aberrantly activated signal transduction pathways downstream of mutated genes. RAS and BRAFV600E mutations constitutively activate the Ras Association Domain Family Member 1 (RASSF1)_mammalian STE20-like protein kinase 1 (MST1) - FOXO3 signaling pathway, and MEK1/2ERK1/2 pathway $(36,43,383)$.

Although the initiation of thyroid cancer is well described in the literature, the progression toward more aggressive metastatic phenotypes is more complex, affecting simultaneously many signaling pathways. Incurable ATC pathogenesis is associated with the activation of platelet-derived growth factor receptor (PDGFR), VEGFR, EGFR, hepatocyte growth factor receptor (HGFR, c-MET), and proto-oncogene c-KIT (CD117), a type III receptor tyrosine kinase. The activation of cell membrane signaling molecules then causes persistent phosphorylation of downstream cell survival, angiogenesis, antiapoptotic, and cell proliferation, supporting PI3K-AKT and MAPK signaling $(364,383)$. The PI3K-AKT pathway is activated by a mutation in $P I 3 K \alpha$ encoding the catalytic p1 $10 \alpha$ subunit or in the PTEN phosphatase gene that functions as a tumor suppressor regulating PI3K activity.

The PI3K-AKT pathway plays a central role in the development and progression of follicular thyroid carcinoma, which is characterized by vascular and capsular invasiveness. In capsular invasion, thyroid tumor cells invade into the peritumoral connective tissue serving as a marker of benign to malignant progression $(104,383)$. Mutations affecting the pathway are less common in follicular thyroid adenoma than in follicular thyroid carcinoma, and therefore, mutations affecting PI3K activity may promote the conversion of adenoma cells to carcinoma cells. The role of NADPH NOX enzymes is poorly characterized in the development 
of follicular thyroid cancer, although PI3K-AKT pathway activation is required for the phosphorylation of $\mathrm{p} 47^{\text {phox }}$ initiation of NADPH NOX assembly, suggesting a role for ROS in the benign to malignant transformation, invasion of cancer cells, and metastatic cancer niche formation $(45,57$, $122,253,334,383)$. As mentioned previously in this review, SOD2 overexpression induces cell migration by activating p130Cas (BCAR1), consequently causing the inactivation of PTEN (127). Interestingly, p130Cas has been shown to interact with thyroid hormone receptor interactor 6 (TRIP6), causing increased MMP expression and the initiation of the invasion program (390). Therefore, signal transduction pathways driving thyroid carcinogenesis may simultaneously upregulate ROSproducing enzymes that participate in cancer progression.

In thyroid tumors, similar to most solid tumors, the paracrine secretion from the stroma is involved in the regulation of epithelial cancer cell growth and migration. Stromal cells secrete a high number of cytokines, growth factors, and ROS that contribute to fibroblast differentiation to activated myofibroblasts, a cornerstone of fibrotic stroma development. An interesting characteristic of PTC stroma is the coexistence of dense desmoplastic stromal regions and highly vascularized inflammatory cell-rich regions (275).

Inflammation is characteristic of highly vascularized stroma, whereas fibrosis and poorly vascularized connective tissue-rich desmoplasia commonly represent the last stromal developmental stages. Increased inflammatory cell migration is characteristic of developing tumors and, in some cases, may even be a cause of malignant transformation. Inflammatory cells secrete a high number of cytokines, growth factors, and ROS, which support epithelial cancer cell growth and tumor microenvironment maturation by regulating growth, migration, and differentiation $(35,40)$.

Although chronic inflammation is associated with premalignant lesion development, the inflammation itself cannot be considered tumorigenic. Instead, the recruitment of inflammatory cells, such as cytotoxic T cells and natural killer (NK) cells, to developing cancer, may represent a failed mechanism of an attempt of the tissue to defend itself against tumorigenesis. Indeed, a recent development of immunotherapy by disrupting the connection of programmed death 1 (PD-1), expressed by $\mathrm{T}$ cells, and the ligand programmed death ligand 1 (PD-L1) and programmed death ligand 2 (PD-L2), expressed by cancer cells, disrupts a crucially important signaling cascade meant to prevent tumor escape from immune destruction (79). In the later phases of carcinogenesis, cancer cells have been shown to educate inflammatory cells toward the protumorigenic mode, indicating the ability of cancer cells to adapt and utilize host tissue defense functions (46).

As part of the last developmental phase of granulation, highly vascularized stroma is gradually replaced by poorly vascularized fibrotic tissue largely composed of collagen secreted by myofibroblasts. NAPDH NOX4-derived ROS have been shown to play a major role in the differentiation of fibroblasts into myofibroblasts, which have been shown to originate from fibroblasts or directly from MSCs (206, 275). Myofibroblasts express MMPs that can remodel the desmoplastic stroma, thus facilitating the local migration of cancer cells and metastasis to distant organs. NADPH NOX1 and especially SOD2 have been shown to stimulate MMP expression and to interrupt focal adhesions as part of the migration/invasion process in cancer progression. In thyroid cancers, fibrotic reaction and desmoplasia correlate with lymph node metastasis and are, therefore, used as a clinical diagnostic marker. Interestingly, desmoplasia develops in the relatively early phase of thyroid tumorigenesis, being present even in $80 \%$ of medullary thyroid cancers and correlating with increased ROS production and increased redox gene expression in stromal cells. Importantly, primary tumor stroma and metastasis site microenvironment are under constant development as the stroma responds to local epithelial cancer cell needs-nurturing the growth and migration requirements. Thus, the tumor stroma contains different developmental stages from early-stage vascular permeability and extravasation of fibrinogen to late-phase increased myofibroblast collagen production $(177,178,317)$.

\section{B. NOX1-5 and SOD1-3 expression in thyroid cancer}

Interestingly, ROS, especially $\mathrm{H}_{2} \mathrm{O}_{2}$, are produced in the thyroid at levels that are normally toxic to cells. Dual oxidases (227) and NOX complexes have been reported to be the main sources of $\mathrm{H}_{2} \mathrm{O}_{2}$ in the thyroid, although the relative expression of NOX1-5 is markedly lower than that of SOD1-3 enzymes. The expression levels of NOX1-5 and SOD1-3 genes in the normal thyroid, follicular adenoma, follicular carcinoma, oncolytic adenoma, oncolytic follicular carcinoma, papillary carcinoma, and anaplastic thyroid carcinoma extracted from the Oncomine Giordano database (109) (Fig. 9) demonstrate the relatively low expression of NOX1-5.

The average NOX1 Log2 median-centered intensitynormalized expression in the normal thyroid is -0.77 and remains at that level in thyroid tumorigenesis showing -0.75 and $-0.85 \log 2$ median-centered intensity-normalized expression in papillary thyroid and anaplastic thyroid cancers, respectively. The normal thyroid average NOX2 $\log 2$ median-centered intensity-normalized expression $(-0.26)$ is significantly $(p<0.01)$ increased in anaplastic thyroid cancer that characteristically has massive inflammatory cell infiltration, explaining the presence of hematopoietic cell NOX2. The average NOX $3 \log 2$ median-centered intensitynormalized expression is extremely low in the thyroid, reflecting its unique role in the regulation of the differentiation of progenitor cells (269).

The association of NOX3 expression with primitive cells is further corroborated by observations suggesting modestly increased mRNA in undifferentiated ovarian teratocarcinoma and adenocarcinoma cancer cells (50). However, the corresponding $\log 2$ median-centered intensity expression for $N O X 3$ in the normal thyroid is -0.87 and remains at that level even in undifferentiated anaplastic thyroid cancer. NOX4, which has a prominent role in cancer and tumor stroma progression, shows an increased average $\log 2$ mediancentered intensity in thyroid tumorigenesis. The expression of NOX4, which in the normal thyroid is -0.37 similar to the level of $N O X 2$, shows a moderate but significant increase in follicular carcinoma $(-0.22 ; p<0.05)$, oncolytic adenoma $(-0.15 ; p<0.05)$, and especially in papillary thyroid carcinoma $(0.04 ; p<0.01)$, characterized by desmosplastic stroma development (Fig. 9).

Interestingly, the database has suggested relatively high SOD1 and SOD3 gene expression in general in normal thyroid tissue and thyroid tumors. The average expression for SOD1 shown as $\log 2$ median-centered intensity in the 

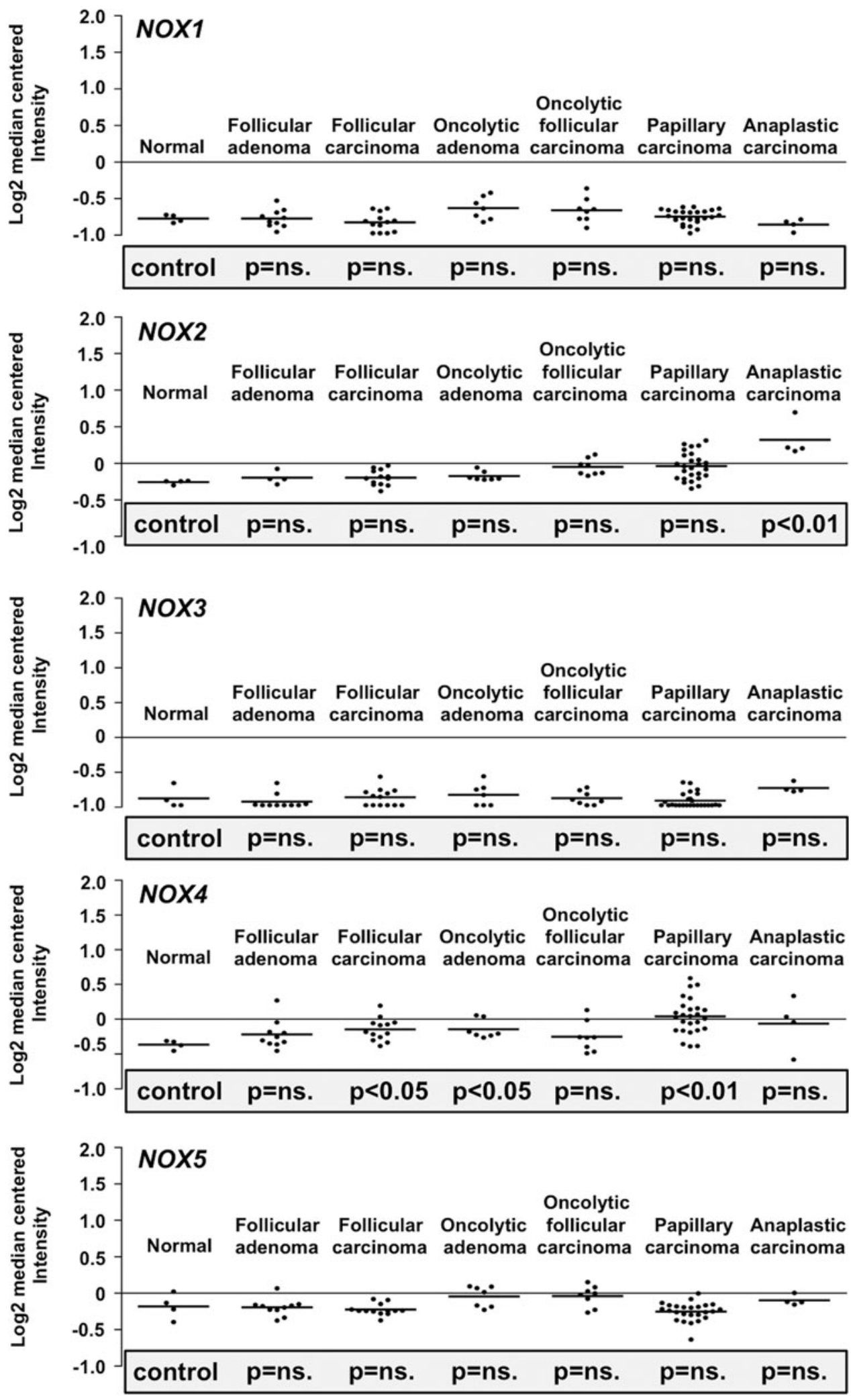

FIG. 9. NOX1-5 and SOD1-3 gene expression in thyroid cancers extracted from Oncomine Giordano database. Gene expression values were collected individually from the database in each thyroid cancer and presented as $\log 2$ mediancentered intensity expression. The $p$-values $(p<0.05$, $p<0.01, p<0.001)$ were determined by two-tail independent samples $t$-tests comparing to normal thyroid expression level. normal thyroid is 1.46 and remains at the same level in thyroid tumors. The average SOD3 Log2 median-centered intensity in the normal thyroid is 0.66 , which is then decreased gradually, showing the lowest values in PTC $(0.15 ; p<0.001)$ and in anaplastic thyroid cancer $(0.18 ; p<0.001)$. The average Log2 median-centered intensity-normalized SOD2 ex- pression in the normal thyroid is markedly lower, -0.34 , than that of SOD1 and SOD3 but different from these two because $S O D 2$ expression gradually increases, correlating with the malignancy of thyroid cancer. There is a moderate increase in follicular adenoma and in the corresponding adenocarcinoma, as well as in oncolytic adenoma and in the 


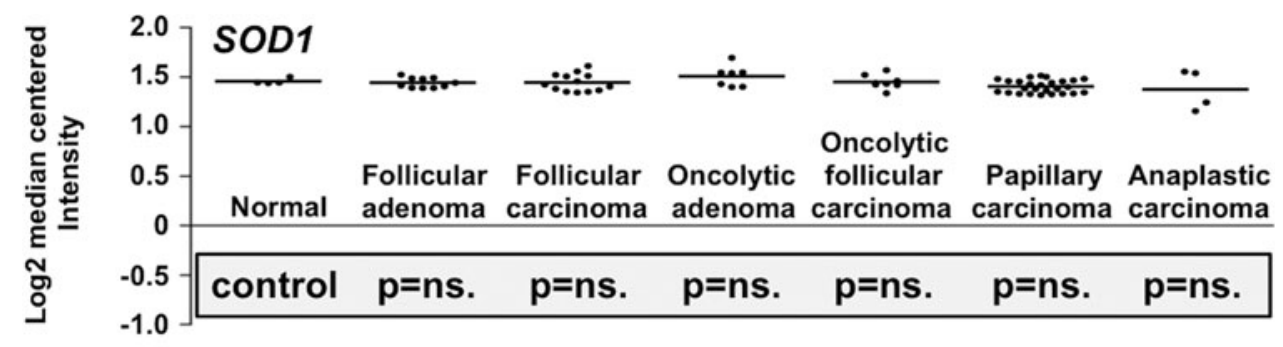

FIG. 9. (Continued).
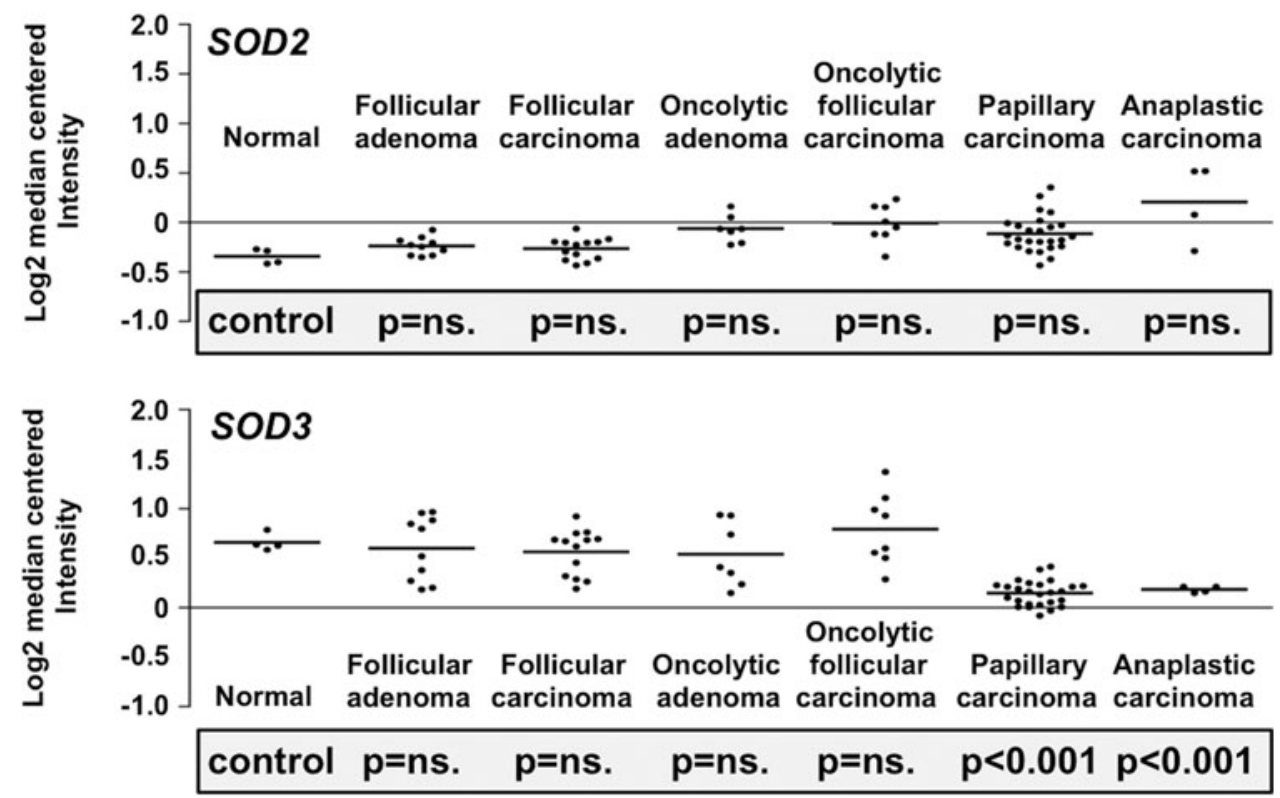

corresponding oncolytic follicular carcinoma. The highest increase in SOD2 expression (0.21) is observed in undifferentiated anaplastic carcinoma, a highly aggressive metastatic tumor. The markedly increased expression of SOD2 in metastatic anaplastic thyroid cancer may be causally related to cancer-related modifications of tumor cell metabolism. The high overall expression levels of SOD1 and SOD3 may instead indicate significant cytoplasmic and extracellular $\mathrm{H}_{2} \mathrm{O}_{2}$ production (Fig. 9).

\section{Percentage change in redox gene expression in PTC}

In general, the gene expression levels vary significantly among individual persons, thus requiring a high number of patients in each study group or alternatively a control sample from the same patient to make definitive conclusions. To confirm the data extracted from Giordano's thyroid database, we normalized mRNA expression in PTC against normal tissue isolated from the same patient using Oncomine's He's database (124). The Log2 median-centered intensity data presented in Figure 10 are supported by the analysis of the percentage increase of NOX1-5 and SOD1-3 in PTC compared with normal thyroid expression levels. In He's database, there were nine patients who had both normal and papillary thyroid tissues available for the expression analysis. The percentage of NOX1-5 expression in PTC compared with normal tissue expression levels suggests a $37.5 \%$ (standard devation [SD] 131.4) increased expression for
NOX1 and a noticeable 532.5\% (SD 513.7) increase for $N O X 2$, indicating PTC inflammatory cell infiltration. The average expression of $N O X 3$ was at the level of normal thyroid (5.2\% increase [SD 9.9]), whereas NOX4 showed a marked $80 \%$ (SD 51.4) average increase from the basic expression levels. NOX5 expression demonstrated a moderate $17.6 \%$ (SD 27.4) increase in PTC tissue (Fig. 10). The

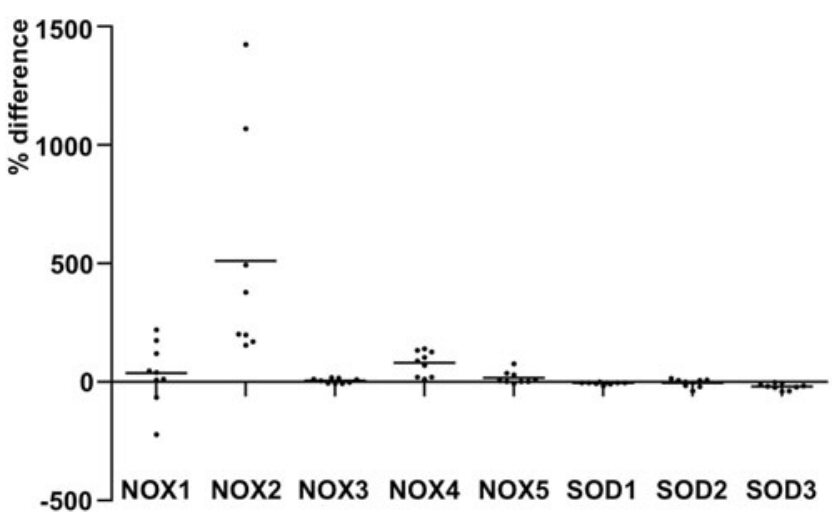

FIG. 10. NOX1-5 and SOD1-3 gene expression in papillary thyroid cancer extracted from Oncomine He database. Gene expression values in papillary thyroid cancer were compared with the normal thyroid expression values of the same patients. The results are shown as percentage change of the $\log 2$ median-centered intensity expression level. 
differences strengthen the observation suggesting high variation among individuals, obligating the use of many patients in each study to convincingly conclude the expression data and, more importantly, redox enzyme-derived effects on downstream signal transduction pathways.

$S O D 1$ expression was decreased in eight of nine patients, $S O D 2$ expression was decreased in four of nine patients, and SOD3 expression was decreased in all nine patients. Therefore, the final conclusions in estimating redox gene expression in patient samples should be associated with the consistency of gene expression. The comparison of the average SOD1, SOD2, and SOD3 mRNA synthesis in normal and PTC showed 4.6\% (SD 4.9), 3.5\% (SD 17.1), and 18.6\% (SD 13.1) decreased expression in cancer, respectively (Fig. 10). Although SOD1 and SOD2 expression levels in He's database are opposite compared with those in the Giordano's database, the differences can be considered minor and might fit between markedly high SDs. Decreased SOD3 is detected in both databases, confirming the earlier observations (184) and suggesting SOD3 as a differentiation marker in thyroid tumorigenesis.

Thus, the Oncomine Giordano and He database results showing thyroid cancer-related redox gene expression suggest that $N O X 1, N O X 3$, and NOX5 mRNA levels do not markedly change in tumorigenesis, whereas NOX2 and NOX4 expression showed increased levels correlating with the degree of transformation. NOX2, which is associated with inflammatory cells, is mostly increased in oncolytic follicular carcinoma, papillary thyroid carcinoma, and, especially, in anaplastic thyroid carcinoma. Of note, anaplastic thyroid carcinoma is marked by the constitutively activated PI3KAKT pathway, which is needed for $\mathrm{p} 47^{\text {phos }}$ NOX2 subunit phosphorylation and NADPH NOX2 complex assembly (383). NOX4 expression is moderately increased in all thyroid tumors, which may be related to the role of the tumor stroma paracrine effect. Indeed, NOX4-produced ROS have been reported to induce fibroblast differentiation to myofibroblasts, thus indicating a growth supportive role for NOX4 in thyroid carcinogenesis.

\section{ROS in Colon Cancer}

\section{A. Progression of colon cancer}

Colon cancer, similar to thyroid cancer, is a wellcharacterized disease, which often progresses from benign adenoma to malignant adenocarcinoma and gains oncogene mutations influencing signal transduction, thereby altering redox gene expression. Colon tumorigenesis is caused by genotoxic stress-derived protumorigenic DNA mutations, activation of oncogenes, and inactivation of tumor suppressor genes in which ROS have a contributory function $(37,116$, 313). Inherited conditions, such as hereditary nonpolyposis colorectal cancer (HNPCC) and familial adenomatous polyposis (FAP), markedly increase the sensitivity to develop malignant carcinoma. Colon tumorigenesis can be divided into three different phases based on the abnormalities observed in colon epithelial cells: initiation, promotion, and progression. In the initiation phase, epithelial cells accumulate DNA damage spontaneously or by the action of protumorigenic factors that then drive the development of aberrant cystic foci, increasing colon cancer incidence. Characteristically, these early events include inactivating mutations in the adenomatous polyposis coli $(A P C)$ tumor suppressor gene that controls growth-promoting WNT signaling by forming the $\beta$-catenin destruction complex together with axis inhibition protein (AXIN), CK1, glycogen synthase kinase $3 \beta(\mathrm{GSK} 3 \beta)$, causing cytoplasmic degradation of $\beta$ catenin (251). Mutations in $A P C$ result in the disrupted function of the $\beta$-catenin destruction complex, increased accumulation of $\beta$-catenin in the cytoplasm, and eventually increased $\beta$-catenin translocation into the nucleus. In the nucleus, $\beta$-catenin interacts with $\mathrm{T}$ cell factor/lymphoid enhancer binding factor (TCF/LEF), inducing the expression of WNT target genes, such as CYCLIN D1 and $c M Y C$ (42). Recently, the importance of changes in colon microbiota has been suggested to increase cancer risk by producing bacterial toxins, such as colibactin. $\mathrm{ROS} \mathrm{O}_{2}{ }^{\bullet-}$ synthesized by Escherichia coli and Enterococcus faecalis is spontaneously dismutated to $\mathrm{H}_{2} \mathrm{O}_{2}$ in the colon environment increasing oxidative stress and consequent DNA base modifications, DNA-protein crosslinks, and DNA single- or double-strand breaks. Colibactin secreted by $E$. coli can cause transient DNA damage, incomplete DNA repair, anaphase bridges, and chromosomal aberrations in mammalian cells even after a short exposure (66).

In the promotion phase, intestinal cells form benign, early, intermediate, and late adenomas. A recent work demonstrated the recruitment of ERK5 signaling in colon carcinoma HCT116 cells to compensate for decreased ERK1/2 kinase phosphorylation allowing the progression of cell proliferation and tumor formation. Although the mitogenic ERK1/2 pathway is the main proliferative signaling route, the study suggested incomplete intestinal epithelial cell maturation and migration along the crypt/villus axis in the absence of phosphorylated ERK1/2, indicating the importance of ERK1/ 2 for normal colon function (71).

Benign colon tumors, such as polyps, can be either mushroom-shaped protrusions inside the large intestine or flat growths in the intestinal wall. Most of the polyps are harmless, but the risk of protumorigenic mutations increases correlating with the size of the polyp. Diminutive $(1-5 \mathrm{~mm}$ in size) and small (6-9 $\mathrm{mm}$ in size) polyps are virtually always benign, whereas large polyps over $10 \mathrm{~mm}$ diameter have an increased tendency to accumulate carcinogenic characteristics. Although polyps are common, the frequency to have carcinoma progression from polyps is low, $\sim 1 \%$ of cases. However, in the case of HNPCC and FAP, the risk of malignant transformation is markedly higher, approximately $50-60 \%$ in HNPPC patients and over $90 \%$ in FAP patients, correlating with the age of the person (115).

In the third progression phase, colon cells in the polyps that have gained a carcinogenic phenotype initiate hyperproliferation, initiating colon cancer progression. Although the nonmetastasized adenocarcinoma can be surgically removed, occasionally it develops into metastatic colon cancer. The mechanism underlying the progression of primary colon adenocarcinoma to metastatic cancer is not completely understood; thus, the disease remains highly lethal with a median survival of 20-36 months. Metastatic cancer progression has been shown to commit mutations affecting proto-oncogenes, especially those of $K R A S, N R A S$, and $B R A F$ that promote cellular migration and invasion, causing characteristic metastasis to the liver, lung, bone, and brain. The overall survival is correlated with the mutations observed - for example, BRAFV600E mutation predicts 
nodal and peritoneal metastasis and a poor prognosis (355). The accumulation of genetic lesions in tumor suppressor gene TP53 and the switch of the tumor suppressor TGF $\beta$ R-SMAD pathway into a tumor promoter by mutation in SMAD4 are further drivers of tumor progression, indicating the complex nature of the disease (152). Other noteworthy mechanisms promoting cancer cell migration include EGFR downstream activator son of sevenless (SOS) and growth factor receptor-bound protein 2 (GRB2) that increases PI3K-AKT signaling (152).

\section{B. WNT signaling in the normal colon and in colon cancer development}

Int/Wingless (WNT) signal transduction is crucial in the maintenance of colon stem cells and progenitor cells, which then differentiate into colon epithelial cells. In general, WNT ligand signals through a canonical pathway regulating $\beta$ catenin entry into the nucleus and through the noncanonical RAS-ERK1/2 and $\mathrm{WNT} / \mathrm{Ca}^{2+}$ pathways stimulating $\mathrm{Ca}^{2+}$ related signaling molecules CAMKII, PLC-PKC, and calcineurin. Activation of canonical signaling contributes to fibroblast activation and fibrosis development, although it has been demonstrated to cause the development and progression of cancer in various model systems (42).

The canonical signal transduction is initiated by WNT ligand binding to the transmembrane frizzled (FZD) receptor, which destabilizes the $\beta$-catenin destruction complex allowing $\beta$-catenin accumulation into the cytoplasm and consequent entry into the nucleus. Downregulation of casein kinase 2 (CK2), which is a positive regulator of WNT signaling and is required for WNT ligand secretion from WNTproducing cells (70), induces PI3K-AKT phosphorylation with consequent phosphorylation and inactivation of FOX$\mathrm{O} 3 \mathrm{a}$ in colon cancer cells. According to previous publications, phosphorylated FOXO3a is translocated from the nucleus into the cytoplasm, inactivating the mRNA synthesis of the target genes SOD1, SOD2, and catalase but also increasing mir21 expression, which targets SOD3 mRNA 3'UTR, resulting in degradation of mRNA $(184,279,363$, 396). Therefore, downregulation of CK2 and consequent impaired WNT signaling may markedly influence the accumulation of $\mathrm{O}_{2}{ }^{\bullet-}$ into cells, likely resulting in colon cancer cell proliferation, survival, and senescence.

The noncanonical $\beta$-catenin-independent signal transduction pathway recruits small GTPase RAC1 and RHOA regulating cytoskeletal remodeling and cell motility via ROCK and JNK activation, whereas the noncanonical $\mathrm{WNT} / \mathrm{Ca}^{2+}$ pathway controls cell migration and is involved in inflammation and in cancer promotion. Noncanonical $\mathrm{WNT} / \mathrm{Ca}^{2+}$ dependent signal transduction can activate PKA/CREB and p38/activating transcription factor 2 (ATF2) signaling molecules or, more interestingly, the PI3K-AKT pathway, which is involved in NADPH-NOX p47 $7^{\text {phox }}$ activation (180). This is supported by observations demonstrating NOX1 participation in the recovery from colon inflammation injury, increasing cell proliferation, migration, terminal differentiation, and antiapoptotic activity $(159,164)$.

\section{NOX1-5 and SOD1-3 gene expression in colon tumorigenesis}

We utilized the Oncomine Sabates-Bellver database (305) to define redox gene expression in benign colon adenoma clinical samples (Fig. 11A). To diminish the variation among individuals, we calculated the percentage difference in gene expression in adenoma tissue compared with normal tissue expression level in the same patient. Interestingly, the average $N O X$ mRNA expression showed a high degree of variation among individuals. The average $N O X 1$ and $N O X 2$ expressions in a benign colon tumor suggested a $16.9 \%$ (SD 13.66) increase and a $4.8 \%$ decrease (SD 65.3), respectively. However, the remarkable high SDs invalidate all definitive conclusions of NOX2 expression levels in colon tumorigenesis. Similar outcome revealed NOX3, NOX4, and NOX5 mRNA expression levels with a $4.3 \%$ increase, a $9.4 \%$ decrease, and a $48.7 \%$ decrease in expression, respectively. The SDs then nullify the average expression data showing SD 44.2 for $N O X 3$, SD 119.5 for NOX4, and SD 185.7 for $N O X 5$ expression in different patients.

As shown in Figure 11A, SOD1 mRNA expression in colon adenoma is increased by $6.2 \%$ (SD 5.2), indicating relatively stable $S O D 1$ expression in the early phase of tumorigenesis. SOD2 expression is increased by $7.6 \%$ (SD 19.2) and SOD3 by $57.3 \%$ (SD 43.5), suggesting a correlation between SOD3 expression and benign tumorigenesis. Similarly, in adenoma development-related $N O X 1-5$ and $S O D 1-3$ gene expression, the progression of primary colon adenocarcinoma to metastatic cancer shows large variation in redox gene expression in individual patients compared to the normal colon tissue values.

The Ki (167) database reporting gene expression in the normal colon, adenocarcinoma, and metastatic colon cancer (Fig. 11B) does not show any values for NOX1 or NOX3 expression, but NOX2 expression suggests relatively stable mRNA levels, showing, on average, a 16.9\% (SD 60.0) increase in the primary tumor and a $19.0 \%$ (SD 74.0) decrease in metastasis (Fig. 11B). NOX4 expression is approximately at the levels of normal tissue, suggesting a mild $11.6 \%$ (SD 146.8) average increase in primary colon carcinoma, whereas there is an average increase of $178.3 \%$ (SD 244.7) in metastasis. NOX5 mRNA expression suggests minimal average changes showing an $8.4 \%$ (SD 256.9) decrease in primary cancer and a $26.3 \%$ (SD 317.4) increase in metastasis (Fig. 11B).

The database suggests increased overall mRNA synthesis for SOD1 and SOD2 in adenocarcinoma (142\% and $77.1 \%$, respectively), although the variations between patients are high as observed in adenoma development: SD 347.4 for SOD1 and SD 108.1 for SOD2. The metastatic disease shows a $515 \%$ increase in SOD1 expression and $73.2 \%$ increase in SOD2 expression; however, again, the SDs, SD 972.6 for SOD1 and SD 108.1 for SOD2, nullify the individual values.

Based on the Oncomine data, the average SOD3 expression level is mildly increased by $14.8 \%$ (SD 121.0) in primary colon carcinoma tumor and moderately decreased by $46 \%$ (SD 136.5). Again, the variation among individuals nullifies the general conclusions of the expression levels; therefore, the effect of SOD3 in tumor progression cannot be generalized using patient expression data alone. However, when evaluating the patient data, it is important to note that several studies have suggested a role for SOD2 in the promotion of cancer cell migration and invasion $(126,165,207)$. The extremely high SDs could causally indicate differences in the signal transduction pathway activation status in patients. Hence, any conclusions for redox gene expression-related data in the colon environment require accurate analysis of the 


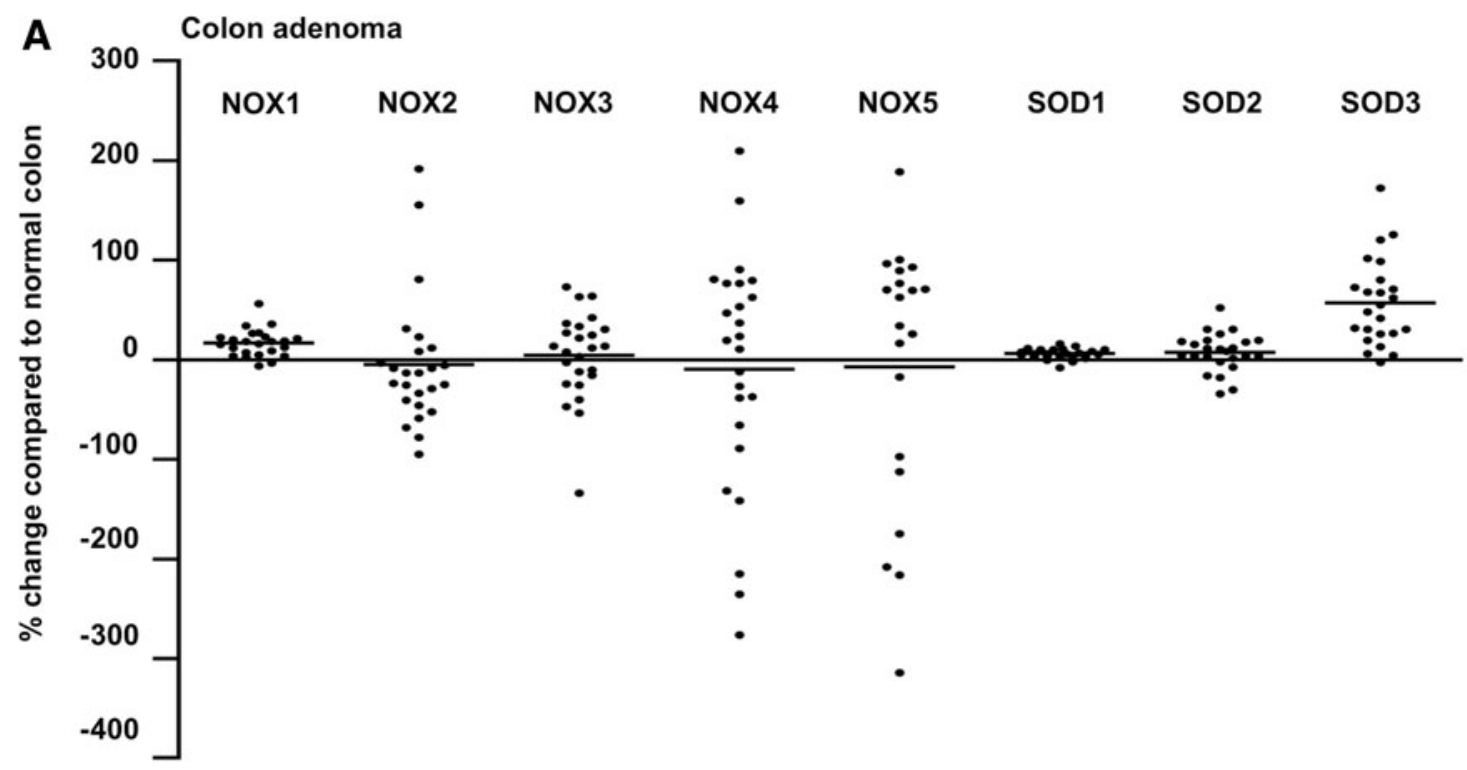

B

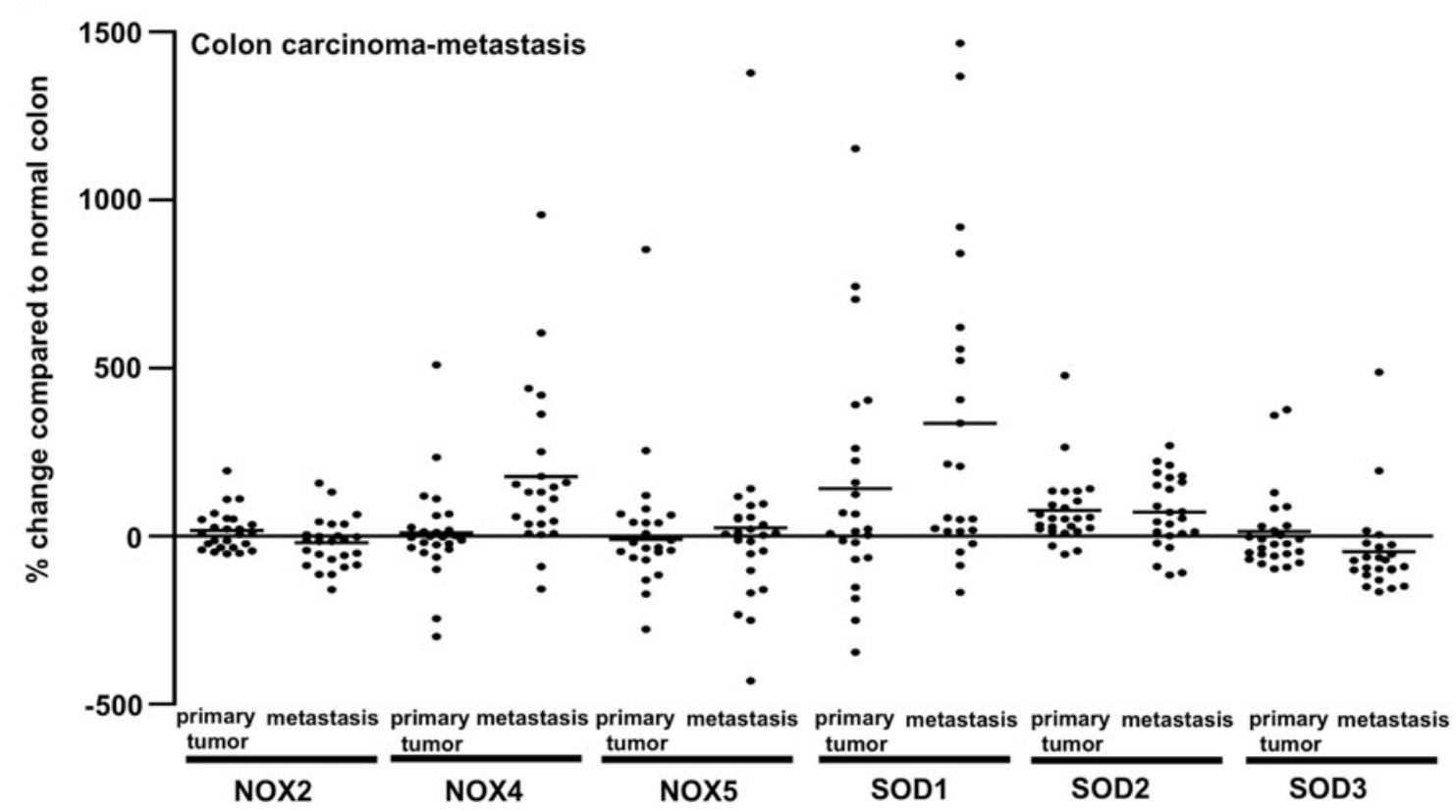

FIG. 11. NOX1-5 and SOD1-3 gene expression in colon tumors extracted from Oncomine Sabates-Bellver and Ki databases. (A) NOX1-5 and SODI-3 expression in benign colon adenoma normalized against the normal colon tissue of the same patient. The data suggest high differences between individuals in redox gene expression. (B) NOX1-5 and SOD1-3 expression in primary colon adenocarcinoma tumor and in metastasis extracted from Ki colon database. Database analysis suggests increased SOD1, NOX4, and NOX5 expression in metastasis, stable SOD2 expression, and decreased SOD3 and NOX2 expression in metastasis.

tumor developmental stage, signal transduction pathways, possible effect of inflammation and microbiota, and an adequate number of patients. Therefore, expression data alone from clinical patients do not comprehensively predict the role of the enzyme in cancer progression. This is further supported by recent data showing increased carcinogenesis in cells with restored SOD3 expression. Virus-mediated gene transfer of SOD3 to restore the expression of the enzyme is correlated with increased primary tumor growth and metastasis levels (368).

\section{ROS in Breast Cancer}

\section{A. ROS-related characteristics of breast cancer}

Breast cancer is frequently classified into four molecular subtypes: (i) luminal A subtype, which has high estrogen and progesterone receptor expression but low human epidermal growth factor receptor 2 (HER2) expression; (ii) luminal B subtype, which has lower estrogen and progesterone receptor levels and variable HER2 expression; (iii) basal-like triple negative subtype, which has negative estrogen receptor, 
progesterone receptor, and HER2 expression; and (iv) HER2 subtype, which has high HER2 expression but generally does not express estrogen and progesterone receptors (9). Breast cancer risk factors are frequently associated with oxidative stress-linked phenomena, such as aging, obesity, smoking, or alcohol consumption, and the pedigree of patients, which is the basis for the hereditary breast/ovarian syndrome that can be caused by mutations in BREAST CANCER 1 (BRCA1), $B R C A 2$, and TP53 tumor suppressor genes $(215,238)$. Breast tumor expansion causes glucose deprivation, hypoxia, and oxidative stress due to lack of blood vessels. Elevated ROS production augments IL-8 and VEGF synthesis in breast cancer cells and enhances MMP secretion, supporting new blood vessel growth and cancer cell migration in the tumor microenvironment and consequently increasing the risk of breast cancer cell intravasation and metastasis $(32,83)$.

Tumor-associated macrophages are frequently observed in breast tumors and contribute to increased ROS production, directly or indirectly, by secreting ROS production-augmenting cytokines, thereby further increasing cancer cell migration, vascularization, and even relapse after chemotherapy treatment (143). Interestingly, a recent study identified a subpopulation of macrophages expressing mannose receptor C-type lectin (MRC1), angiopoietin receptor TIE2, VEGF-A, and CXCR4 in densely vascularized regions of breast tumors following chemotherapy; this finding suggests a potential role of macrophages in disease relapse (143).

As reported in other cancers, ROS are powerful DNA damaging agents that cause strand breaks, chromosomal rearrangements, and individual DNA base modification. In breast cancer, germ line mutations in $B R C A 1$ and $B R C A 2$ are examples of genomic instability that increases tumor susceptibility (257).

Previous studies have demonstrated upregulation of the expression of the NADPH oxidases NOX1, NOX4, and NOX5 in breast cancer, suggesting mitochondrial $\mathrm{H}_{2} \mathrm{O}_{2}$ production by NADPH oxidase NOX4, which in turn protects cancer cells against etoposide cytotoxin-induced cell death, increases invasion, anchorage-independent growth, and EMT $(28,113)$. Other studies have suggested that NADPH oxidase NOX4 promotes carcinogenesis and protects breast cancer cells from anoikis. Mechanistically, TGF $\beta$ secreted into the tumor environment causes phosphorylation of the SMAD2/3, SMAD complex and JNK activation, which in turn increases NADPH oxidase NOX4 synthesis. Alternatively, AKT1 phosphorylation may increase SP1 transcription factor synthesis and subsequent NADPH oxidase NOX4 production, which is paralleled with increased $\mathrm{F}$-actin polymerization, invapodia formation, and increased invasion (28, 55, 351, 370).

In conjunction with the NAPDH oxidase NOX4, SOD2 contributes to breast cancer progression at late stage III by promoting AMPK phosphorylation at Thr172. AMPK then directs cellular oxidative respiration toward glycolysis, thus improving cell viability and inhibiting apoptosis (121).

\section{B. NOX1-5 and SOD1-3 gene expression in breast tumorigenesis}

We extracted redox gene expression in patients with normal breast, in situ ductal breast carcinoma, and invasive ductal breast carcinoma from the Oncomine Ma database (210) (Fig. 12A). For the analysis, we included only patients from whom all three tissue types were isolated for the microarray. The analysis indicates relatively stable $N O X 1, N O X 2, N O X 3$, and $N O X 5$ production, suggesting no significant differences at the expression levels between normal and tumor tissues, whereas NOX4 expression is strongly upregulated in both in situ and invasive breast carcinoma. SOD1 has high overall expression in breast tissue, and its expression is further increased in tumor tissues, whereas SOD2 and SOD3 are expressed at approximately levels twofold lower than SOD1 levels.

Although SOD2 expression is statistically at the same level in normal and pathological tissue, the data suggest a decreasing trend in the expression correlating to progression of tumorigenesis. We then calculated the percentage change in the redox gene expression levels in tumors compared to normal breast tissue from the same patient (Fig. 12B-I). Similar to that in thyroid and colon cancers, the individual differences nullified the statistical significance, thus corroborating the notion that redox gene expression alone may not predict carcinogenic progression.

\section{ROS in Lung Cancer}

\section{A. ROS-related characteristics of lung cancer}

Nonsmall-cell lung cancer (NSCLC) and small-cell lung cancer (SCLC) are the primary lung cancer types. NSCLC, which represents $75-80 \%$ of the cases and includes adenocarcinoma, squamous cell carcinoma, and large-cell carcinoma, originates from activating mutations in $E G F R, A L K$, ROS 1 proto-oncogene receptor tyrosine kinase, and $B R A F$ genes. Characteristically, NSCLC may develop resistance to therapy by driver mutations in EGFR, BIM, KRAS, MET, and $H E R 2$ that alter downstream signaling, allowing the tumors to bypass the upstream tyrosine kinase-targeted therapy (304). SCLC, which accounts for $\sim 15 \%$ of lung cancers, is a highly aggressive and highly vascularized metastatic form of cancer characterized by mutations in TP53 and the transcriptional corepressor $R B 1$, disruption of several signaling networks, and overexpression of MYC, MYCL, and MYCN transcription factors. Interestingly, small dense core granules identified as neuroendocrine cells in the tumors secrete adrenocorticotropic hormone, bombesin-like gastrin releasing peptides, and regulatory neuropeptides, thereby making SCLC a neuroendocrine lung tumor $(41,100,191)$.

Although the oncogene mutations identified in lung cancers affect several signaling pathways that regulate redox enzyme expression, the role of ROS in lung carcinogenesis is not completely characterized. Studies performed in lung cancer models have resulted in interesting novel observations, such as NADPH oxidase NOX1-coordinated inhibition of p53 acetylation at Lys382 that affects p53 proapoptotic function; increased NOX2 expression in NSCLC patients, which correlates with decreased survival (293); and a novel role for the NADPH oxidase NOX3 in the regulation of redox balance in NSCLC causing a selective degradation of mutant EGFR (204). As reported previously, upregulation of the NADPH oxidase NOX4 contributes to myofibroblast activation and has a statistically significant correlation to patient survival (119). In addition, a recent study identified SOD1 as a tumor promoter in NSCLC, demonstrating that SOD1 inhibition with ATN-224 induces cellular death (110, 158, 274). Therefore, NADPH oxidases $1-5$ and SOD1-3 potentially have prominent roles in lung carcinogenesis. 

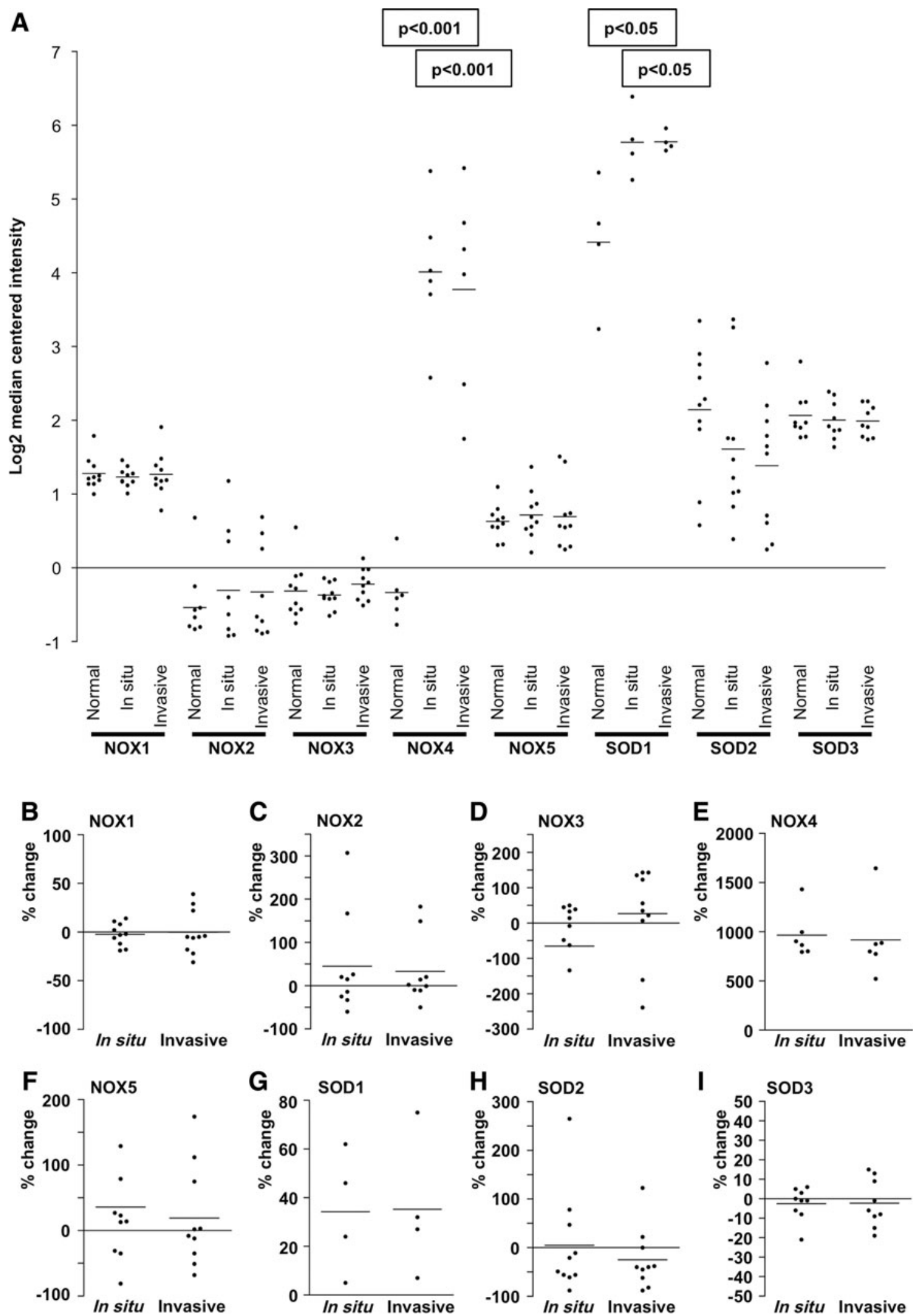

FIG. 12. NOXI-5 and SODI-3 gene expression in breast tumors extracted from the Oncomine Ma database. (A) NOX1-5 and SOD1-3 expression in normal breast, in ductal breast carcinoma in situ, and in invasive ductal breast carcinoma of the same patient. NOX4 expression is significantly $(p<0.001)$ increased in both in situ and invasive ductal breast carcinomas. (B-I) Percentage change in NOXI-5 and SODI-3 expression in ductal breast carcinoma in situ and in invasive ductal breast carcinoma as normalized against the normal breast tissue of the same patient. $p$-Values $(p<0.05$, $p<0.01, p<0.001$ ) were determined by two-tail independent sample $t$-tests. 


\section{B. NOX1-5 and SOD1-3 gene expression in lung tumorigenesis}

The analysis of the Oncomine Landi database (186) suggested no differences in NOX1-3 and NOX5 expression levels between normal and adenocarcinoma lung tissues (Fig. 13A), whereas NOX4 expression was significantly increased in tumors. SOD1 had markedly higher mRNA levels compared with $S O D 2$ or $S O D 3$, the latter being significantly downregulated in adenocarcinoma tissues. Unlike that in several other tumors, the expression levels demonstrated relatively minor variation between individual patients. The percentage change in the expression levels compared to the normal lung supports the observation of minor patientspecific differences, excluding the expression of NOX5 (Fig. 13B). Although the total expression levels of NOX5 are low in individual patients (Fig. 13A), the increases or decreases vary from $-192 \%$ to $426 \%$ (Fig. 13B), thereby suggesting that mutations and alterations in signal transduction pathways in lung adenocarcinoma markedly affect NOX5 regulation.

\section{ROS in Hematological Cancers}

Hematological cancer cells are not in constant bidirectional interaction with tumor stroma, therefore representing an alternative model system for ROS studies. Self-renewal capacity, differentiation, aging, and the transformation of the most primitive HSCs are linked to ROS-derived DNA damage suggesting the multifaceted effect of redox enzymes in HSC fate. Interestingly, HSCs have been demonstrated to express several free radical-producing genes (286), although the HSC niche is characterized by hypoxia, which is one of the cornerstones in maintaining the primitive progenitor cell phenotype $(56,205)$. Importantly, ROS have been suggested to be a mediator in cell fate decision, in the induction of primitive hematopoietic progenitor cell differentiation and in the development of hematological malignancies $(132,149$, $225,226)$. In solid tumor development, ROS play a marked role in the induction of primary cell immortalization and transformation $(39,144)$ through the activation of oncogene- induced senescence and in the immortalization/transformation of primary cells. Interestingly, solid tumors have a mosaic senescent phenotype, suggesting the presence of cells that have lost the growth arrest signaling checkpoints-for example, through the downregulation of $\mathrm{p} 21 / \mathrm{p} 19 / \mathrm{p} 16$ signaling pathways (315). Correspondingly, in hematologic malignancies, the accumulation of ROS-derived DNA damage with consequent genomic aberrations has been suggested to demarcate the initiation and progression of the disease (310), although the function of ROS in hematological neoplasias is not completely characterized.

\section{A. ROS in CD34 HSC differentiation}

HSCs, which originate from the mesoderm during ontogeny, differentiate into myeloid blood cell lineages (monocytes/macrophages, erythrocytes, neutrophils, basophils, eosinophils, platelets, and megakaryocytes) and lymphoid cell lineages ( $T$ cells, B cells, NK cells) (265). In mouse models, primitive hematopoietic progenitor cells have been classified into long-term hematopoietic stem cells (LT-HSCs), short-term HSCs, multipotent progenitor cells, and unipotent progenitor cells reflecting their self-renewal ability, cell cycling frequency, and differentiation status $(248,357)$.

Although an emerging number of studies have suggested that ROS and ROS-related factors influence the development, self-renewal, migration, and differentiation of HSCs (145, $148,149,153,286)$, HSCs have been demonstrated to express various redox enzymes $(284,286,353)$ at relatively low levels. Oncomine Valk DNA microarray profiling of CD $34^{+}$ bone marrow and peripheral blood HSCs from patients showed lower $N O X 1, N O X 3$, and NOX4 mRNA expression than NOX2, NOX5, SOD1, SOD2, and SOD3 expression, suggesting efficient $\mathrm{O}_{2}{ }^{\bullet-}$ removal from HSCs (Fig. 14) (362). The hypoxic niche has been shown to coordinate low redox enzyme and ROS levels through hypoxia-inducible factor $1 \alpha$ (HIF-1 $\alpha$ ), a well-recognized redox gene expression regulator (169). Stabilization of cytoplasmic HIF- $1 \alpha$ allows nuclear localization and consequent binding of HIF- $1 \alpha$ to hypoxia response element (HRE) frequently found in the promoter
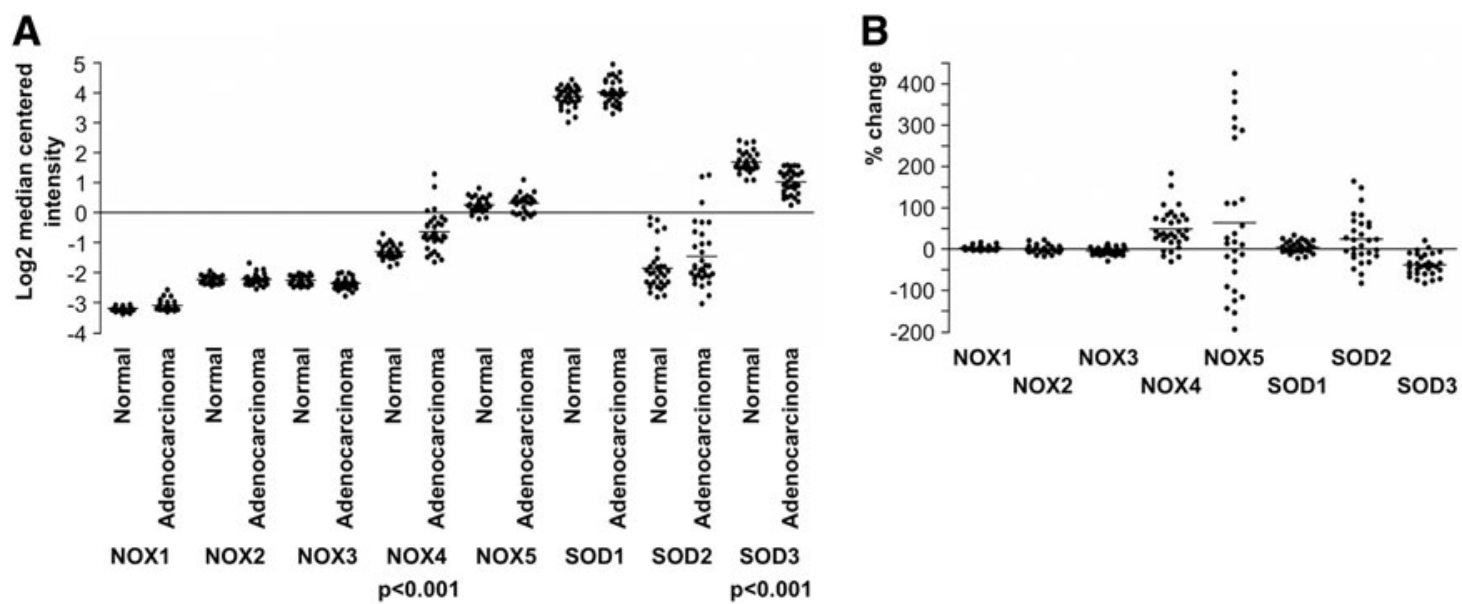

FIG. 13. NOX1-5 and SOD1-3 gene expression in lung tumors extracted from the Oncomine Landi database. (A) NOX1-5 and SOD1-3 expression in normal lung and in lung adenocarcinoma of the same patient. (B) Percentage change of NOX1-5 and SOD1-3 expression in lung adenocarcinoma normalized against the normal lung tissue of the same patient. 


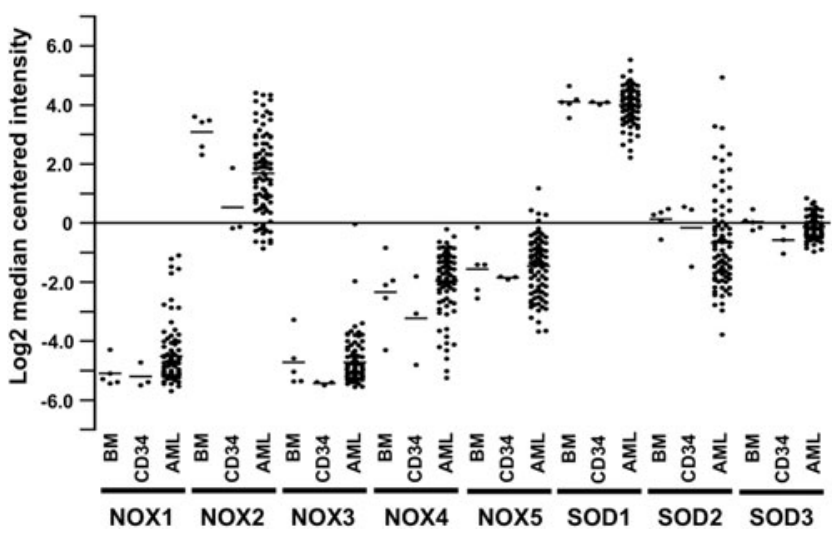

FIG. 14. NOX1-5 and $S O D 1-3$ gene expression in $\mathrm{CD34}^{+}$cells and in AML extracted from Oncomine Valk database. Gene expression values were collected from BM, peripheral blood $\mathrm{CD} 34^{+}$, and AML cells. NOX2, NOX4, NOX5, SOD2, and SOD3 redox gene expression values show differences between hypoxic BM and circulating $\mathrm{CD} 34^{+}$primitive hematopoietic progenitor cells. NOX2 and SOD1 show the highest expression values in collected samples. AML, acute myeloid leukemia; BM, bone marrow.

region of redox genes (285). Physical tissue structures, such as arterial endothelial integrity, have been suggested to regulate the hypoxic environment by allowing or blocking extra- and intravasation of blood cells and serum components. Niches close to permeable sinusoidal arteries contain higher ROS concentrations, which correlate with a higher number of cycling and apoptotic primitive hematopoietic progenitor cells than niches located at less permeable arteries harboring the most primitive LT-HSCs (147).

Although the expression of ROS-producing enzymes is relatively low in the bone marrow HSC niche, it has been suggested that persistent genotoxic stress could lead to DNA damage accumulation in quiescent cells, eventually inducing their differentiation, aging, apoptosis, and malignant transformation $(99,373)$. The hypothesis was corroborated by recent studies, which demonstrated a correlation between increased ROS production, replicative stress, DNA damage, and premature senescence of stem cells in in vivo models. A high ROS content in grafted HSCs correlates with reduced repopulation ability in tertiary recipient mice, whereas HSCs with low ROS levels had significantly higher homing and engraftment capacity $(132,386)$. This was further supported by reports demonstrating that NAC treatment reduced the bone marrow ROS content and increased the human HSC transplantation efficiency in NOD-SCID mice $(141,146)$. Therefore, accumulated DNA damage could function as a physiological indicator of aging, affecting grafted stem cell homing and engraftment $(291,386)$.

Mechanistically, the accumulation of genomic damage is a result of the coordinated action of oxidative injuries, mutations caused by irradiation or chemicals, and aberrant DNA replication. In general, DNA damage activates the DNA damage response (DDR) signaling pathway, which is linked to increased $\mathrm{YH} 2 \mathrm{AX}-\mathrm{ATM}-\mathrm{CHK} 2-\mathrm{p} 53$ DDR signal activation in the grafted cells, leading to the induction of cell cycle arrest with consequent DNA repair, differentiation, apoptosis, senescence, or transformation of HSCs $(132,303,398)$.
Interestingly, DNA damage may accumulate in the G0 phase into LT-HSCs due to low cell cycle frequency and consequent minimal DDR mechanisms, which are dependent on cell cycle-associated DNA replication. In the DNA injury correction process, the phosphorylation of p53 halts at cell cycling checkpoints G1/S and G2/M until DNA repair enzymes have corrected the mutations $(54,356)$. The hypothesis is in line with observations demonstrating markedly increased $\mathrm{H} 2 \mathrm{AX}$ phosphorylation in HSCs isolated from old study subjects $(303,398)$.

Sources of ROS-induced DNA damage and HSC differentiation are incompletely characterized. The contribution of $\mathrm{O}_{2}{ }^{\bullet-}$ and $\mathrm{H}_{2} \mathrm{O}_{2}$ has been linked to the malignant transformation of hematopoietic cells $(135,219,296)$, although $\mathrm{H}_{2} \mathrm{O}_{2}$-producing SOD3 stimulates hematopoiesis by increasing primitive progenitor cell differentiation $(1,97)$. The contribution of SOD3 to HSC differentiation was causally supported by a study demonstrating that FOXO-null mice had reduced long-term repopulation ability. In these mice, sod3 was identified as one of highly expressed leading edge genes (356), supporting the hypothesis that the enzyme can initiate the differentiation of HSCs by inducing DNA damage, maybe followed by activation of the differentiation process or, alternatively, stimulation of cell cycling and differentiation through the mitogenic RAS-ERK1/2 signal transduction pathway.

\section{B. ROS in hematological cancers and therapy}

ROS-derived DNA damage is involved in the gradual progression of carcinogenesis, initiating from evolvement and amplification of self-renewing preleukemic HSC clones and leading to the development of AML or relapse after therapy (151). Mutations in plasma membrane-bound FLT3-ITD, a major genetic lesion leading to AML development, have been demonstrated to cause altered signal transduction, phosphorylation of STAT5, and consequent activation of RAC1, increasing ROS production. ROS-derived inactivation or decreased expression of phosphotyrosine phosphatases, such as SHP-1 and DEP-1/PTRRJ, may then contribute to transformation of 32D mouse hematopoietic progenitor cells and TF-1 human erythroblast $(49,111,245)$.

STAT5 may also augment metabolic activity of myeloproliferative neoplastic cells by increasing the expression of 6-phosphofructo-2-kinase/fructose-2,6-biphosphatase 3 (PFKFB3) and by regulating JAK2V617F-dependent lactate production, metabolic activity, and glucose uptake (298). In chronic myelogenous leukemia (CML) cells, PI3K-AKTGSK3 $\beta$ - $\beta$-CATENIN-MCL1 signaling has been linked to increased survival that directly correlates with increased ROS, especially $\mathrm{H}_{2} \mathrm{O}_{2}$, production. CML is frequently caused by translocation mutation between chromosomes 9 and 22 resulting in the formation of chimeric BCR-ABL kinase, which then stimulates synthesis of NADPH oxidase NOX4. Interestingly, NADPH oxidase NOX4-synthesized ROS inactivate phosphotyrosine phosphatase $\mathrm{PP} 1 \alpha$, thereby activating PI3K-AKT signaling and further strengthening CML cell drug resistance and cancer cell survival (252).

Oxygen radicals, DNA base modifications, and aberrant SOD and CATALASE expression contribute to the progression of chronic lymphocytic leukemia (CLL), acute lymphoplastic leukemia, monoclonal B lymphocytosis 
(MBL), a disease marked by B-cell expansion, and the CLL phenotype, which characteristically show increased levels of 8-oxo-2'-deoxyguanosine (8-oxo-dG) in lymphocytes and in urine. In a recent study, it was shown that the 8-oxo-dG mutation level in $29 \mathrm{MBL}$ patients between 70 and 77 years and in 55 CLL patients between 63 and 77 years was significantly increased compared with that in 31 control patients $(18,60,259,264,319,400)$, therefore supporting the observations suggesting the early contribution of oxidative stress in hematological malignancies.

Further evidence supporting ROS-induced genetic aberrations was demonstrated in a study showing increased frequency of DNA double-strand breaks and nonhomologous genomic rearrangements in a myeloplastic syndrome (MS) mouse model. The authors suggested that ROS-derived genomic instability contributes to the clonal expansion of bone marrow $\mathrm{CD} 34^{+}$cells in $R A S-B C L 2$ mutation-harboring mice modeling MS/AML development. The involvement of ROS was confirmed by NAC treatment, which reversed DNA damage and error-prone DNA damage repair $(296,311)$. NAC primarily neutralizes the $\mathrm{O}_{2}{ }^{\bullet-}$ radical, although it can also react with $\mathrm{H}_{2} \mathrm{O}_{2}$, therefore stressing the role of ROS in carcinogenesis.

However, the data extracted from the Oncomine Valk leukemia database consisting of gene expression data analyzed from 293 patients with AML highlight the expression of $\mathrm{H}_{2} \mathrm{O}_{2}$-producing SOD enzymes (Fig. 14) (362). The data demonstrate stable and low mRNA expression of NOX1, $N O X 3, N O X 4$, and NOX5, whereas the expression of NOX2 is markedly higher and variable in different forms of cancer. Interestingly, the expression of $S O D$ isoforms, especially $S O D 1$, is clearly higher than that of $N O X$ genes in all patient groups, suggesting SOD enzymes as a primary source of ROS in hematological malignancies. In general, the expression of $N O X$ genes is increased in malignancies, whereas the expression of $S O D 1, S O D 2$, and SOD3 is more variable, correlating with the differentiation status of the cells.

Irradiation can have a drastic impact on bone marrow stem cell clonal activity, affecting survival, expansion, mobilization, the ability to reconstitute the bone marrow niches, and differentiation of HSC clones (53, 193, 233, 255). In general, moderate or high irradiation can affect the primitive phenotype of stem cells by inducing differentiation, senescence, and apoptosis, or affecting the integrity of the stem cell niche $(17,77,235)$ by increasing ROS production accompanied by oxidative DNA damage, with activation of ataxia-telangiectasia mutated (ATM)-CHK-p53-p21 and p38 MAPK cascades, the main signaling pathways induced by irradiation $(282,324)$.

The damage to the HSC niche occurs in two different modalities that are mediated by damaged cobblestone area forming colonies and by the disruption of the normal niche phenotype regulating stem cell proliferation and differentiation (179). Therefore, irradiation-derived ROS may have a marked influence on the HSC status on quiescent HSCs, modifying their stemness and even reflecting the aging of HSCs and might play a role in recovery of irradiation therapy patients $(193,208,301,387)$. Although the focus of ROS studies until now has been on DNA damage and the consequences of DNA injury on differentiation, aging, and on transformation of HSCs, ROS can activate per se cell signal transduction pathways, affecting the growth and survival of cells in an autocrine or a paracrine manner. As the HSC microenvironment contains redox enzyme expressing MSCs and/or MSC derivatives $(208,234,241,306)$, the role of the paracrine activity of niche cells could clarify further the role of ROS in HSC self-renewal and differentiation.

\section{Summary and Conclusions}

Determining a universal measurable redox balance in cells is virtually impossible due to a high number of factors influencing the cellular redox state. Involvement of interacting cell signal transduction pathways may nevertheless assist cells to adapt to oxidative stress by coordinating oxidant and antioxidant gene expression, although they may also cause a differential response in cells, representing different diseases or differentiation degrees depending on the activation status of the signaling pathways. ROS production is most noticeably affected by GPCR activation, $\mathrm{Ca}^{2+}$ signaling, and $\mathrm{H}_{2} \mathrm{O}_{2}$-directed modification of the activation level of small GTPase RAC, which is an important subunit in NADPH oxidase NOX1, NOX2, and possibly also in NOX3. ROSactivated PI3K-AKT signaling is another compellingly interesting cascade because it is needed for $\mathrm{p} 47^{\text {phox }}$ phosphorylation, which initiates the NAPDH oxidase complex assembly and might represent a key pathway in balancing $\mathrm{H}_{2} \mathrm{O}_{2}$ and $\mathrm{O}_{2}{ }^{--}$concentrations in cells.

The tissue environment provides additional uncharacterized factors that influence the overall redox gene expression, resulting in markedly high variation among patients. Therefore, the conclusions based on the data obtained from a large patient population may not be directly comparable with the results obtained from a single cell culture. Importantly, in vitro cell models may have limitations in modeling cancer depending on the mutations in oncogenes, activation status of the signaling pathways, and the differences in mechanisms of individual redox gene induction, which then influence redox gene expression. Although the data observed in the cell culture model systems have unquestionably demonstrated the role of $\mathrm{O}_{2}{ }^{--}$ and $\mathrm{H}_{2} \mathrm{O}_{2}$ in tumorigenesis, the differences in the in vitro and in vivo model systems used could explain some contradictory conclusions in the function of redox enzymes in cancer.

When evaluating the data observed in the model systems, it is crucial to distinguish the differential signaling effects of ROS, especially those of $\mathrm{H}_{2} \mathrm{O}_{2}$, which directly correlate to the level of ROS. This, in turn, affects the use of redox genes for diagnostic purposes. Although some redox genes could be considered supplemental candidate markers in carcinogenesis, the individual discrepancy in the mRNA expression levels in patients invalidates their reliability in prognostic diagnosis. However, the systemic identification of signaling pathways affecting simultaneously the activation of redox genes or mediating the ROS effect may offer potential novel drug targets in cancer treatment or complement existing clinical protocols.

\section{Acknowledgment}

This work was supported by the Italian Ministry of Health. This work has been supported by project POR Campania CUP B63D18000210007.

\section{References}

1. Abdel-Mageed AS, Senagore AJ, Pietryga DW, Connors RH, Giambernardi TA, Hay RV, and Deng W. Intravenous administration of mesenchymal stem cells 
genetically modified with extracellular superoxide dismutase improves survival in irradiated mice. Blood 113: 1201-1203, 2009.

2. Abrahamsson T, Brandt U, Marklund SL, and Sjoqvist PO. Vascular bound recombinant extracellular superoxide dismutase type $\mathrm{C}$ protects against the detrimental effects of superoxide radicals on endothelium-dependent arterial relaxation. Circ Res 70: 264-271, 1992.

3. Adachi T, Toishi T, Takashima E, and Hara H. Infliximab neutralizes the suppressive effect of TNF-alpha on expression of extracellular-superoxide dismutase in vitro. Biol Pharm Bull 29: 2095-2098, 2006.

4. Adachi T, Yamada H, Futenma A, Kato K, and Hirano K. Heparin-induced release of extracellular-superoxide dismutase form (V) to plasma. J Biochem 117: 586-590, 1995.

5. Adachi Y, Shibai Y, Mitsushita J, Shang WH, Hirose K, and Kamata T. Oncogenic Ras upregulates NADPH oxidase 1 gene expression through MEK-ERK-dependent phosphorylation of GATA-6. Oncogene 27: 4921-4932, 2008.

6. Alvero AB, Montagna MK, Sumi NJ, Joo WD, Graham E, and Mor G. Multiple blocks in the engagement of oxidative phosphorylation in putative ovarian cancer stem cells: implication for maintenance therapy with glycolysis inhibitors. Oncotarget 5: 8703-8715, 2014.

7. Ambasta RK, Kumar P, Griendling KK, Schmidt HH, Busse R, and Brandes RP. Direct interaction of the novel Nox proteins with $\mathrm{p} 22$ phox is required for the formation of a functionally active NADPH oxidase. $\mathrm{J}$ Biol Chem 279: 45935-45941, 2004.

8. Andersen JK. Oxidative stress in neurodegeneration: cause or consequence? Nat Med 10(Suppl): S18-S25, 2004.

9. Anderson WF, Rosenberg PS, Prat A, Perou CM, and Sherman ME. How many etiological subtypes of breast cancer: two, three, four, or more? J Natl Cancer Inst 106: pii dju165, 2014.

10. Antony S, Wu Y, Hewitt SM, Anver MR, Butcher D, Jiang G, Meitzler JL, Liu H, Juhasz A, Lu J, Roy KK, and Doroshow JH. Characterization of NADPH oxidase 5 expression in human tumors and tumor cell lines with a novel mouse monoclonal antibody. Free Radic Biol Med 65: 497-508, 2013.

11. Aravindan N, Aravindan S, Pandian V, Khan FH, Ramraj SK, Natt $\mathrm{P}$, and Natarajan $\mathrm{M}$. Acquired tumor cell radiation resistance at the treatment site is mediated through radiation-orchestrated intercellular communication. Int $J$ Radiat Oncol Biol Phys 88: 677-685, 2014.

12. Arbiser JL, Petros J, Klafter R, Govindajaran B, McLaughlin ER, Brown LF, Cohen C, Moses M, Kilroy S, Arnold RS, and Lambeth JD. Reactive oxygen generated by Nox1 triggers the angiogenic switch. Proc Natl Acad Sci U S A 99: 715-720, 2002.

13. Azzolin VF, Cadona FC, Machado AK, Berto MD, Barbisan F, Dornelles EB, Glanzner WG, Goncalves PB, Bica CG, and da Cruz IB. Superoxide-hydrogen peroxide imbalance interferes with colorectal cancer cells viability, proliferation and oxaliplatin response. Toxicol In Vitro 32: 8-15, 2016.

14. Bachelder RE, Wendt MA, and Mercurio AM. Vascular endothelial growth factor promotes breast carcinoma invasion in an autocrine manner by regulating the chemokine receptor CXCR4. Cancer Res 62: 7203-7206, 2002.

15. Banfi B, Molnar G, Maturana A, Steger K, Hegedus B, Demaurex N, and Krause KH. A $\mathrm{Ca}(2+)$-activated
NADPH oxidase in testis, spleen, and lymph nodes. $J$ Biol Chem 276: 37594-37601, 2001.

16. Banfi B, Tirone F, Durussel I, Knisz J, Moskwa P, Molnar GZ, Krause KH, and Cox JA. Mechanism of $\mathrm{Ca}^{2+}$ activation of the NADPH oxidase 5 (NOX5). $J$ Biol Chem 279: 18583-18591, 2004.

17. Barazzuol L, Ju L, and Jeggo PA. A coordinated DNA damage response promotes adult quiescent neural stem cell activation. PloS Biol 15: e2001264, 2017.

18. Battisti V, Maders LD, Bagatini MD, Santos KF, Spanevello RM, Maldonado PA, Brule AO, Araujo Mdo C, Schetinger MR, and Morsch VM. Measurement of oxidative stress and antioxidant status in acute lymphoblastic leukemia patients. Clin Biochem 41: 511-518, 2008.

19. Bedard K and Krause KH. The NOX family of ROSgenerating NADPH oxidases: physiology and pathophysiology. Physiol Rev 87: 245-313, 2007.

20. BelAiba RS, Djordjevic T, Petry A, Diemer K, Bonello S, Banfi B, Hess J, Pogrebniak A, Bickel C, and Gorlach A. NOX5 variants are functionally active in endothelial cells. Free Radic Biol Med 42: 446-459, 2007.

21. Benfeitas R, Uhlen M, Nielsen J, and Mardinoglu A. New challenges to study heterogeneity in cancer redox metabolism. Front Cell Dev Biol 5: 65, 2017.

22. Benlloch M, Mena S, Ferrer P, Obrador E, Asensi M, Pellicer JA, Carretero J, Ortega A, and Estrela JM. Bcl-2 and Mn-SOD antisense oligodeoxynucleotides and a glutamine-enriched diet facilitate elimination of highly resistant B16 melanoma cells by tumor necrosis factoralpha and chemotherapy. J Biol Chem 281: 69-79, 2006.

23. Berto MD, Bica CG, de Sa GP, Barbisan F, Azzolin VF, Rogalski F, Duarte MM, and da Cruz IB. The effect of superoxide anion and hydrogen peroxide imbalance on prostate cancer: an integrative in vivo and in vitro analysis. Med Oncol 32: 251, 2015.

24. Bertram A and Ley K. Protein kinase C isoforms in neutrophil adhesion and activation. Arch Immunol Ther Exp (Warsz) 59: 79-87, 2011.

25. Block K, Eid A, Griendling KK, Lee DY, Wittrant Y, and Gorin Y. Nox4 NAD(P)H oxidase mediates Srcdependent tyrosine phosphorylation of PDK-1 in response to angiotensin II: role in mesangial cell hypertrophy and fibronectin expression. J Biol Chem 283: 24061-24076, 2008.

26. Bos JL, Rehmann $\mathrm{H}$, and Wittinghofer A. GEFs and GAPs: critical elements in the control of small $G$ proteins. Cell 129: 865-877, 2007.

27. Boudreau HE, Casterline BW, Burke DJ, and Leto TL. Wild-type and mutant p53 differentially regulate NADPH oxidase 4 in TGF-beta-mediated migration of human lung and breast epithelial cells. Br J Cancer 110: 2569-2582, 2014.

28. Boudreau HE, Casterline BW, Rada B, Korzeniowska A, and Leto TL. Nox4 involvement in TGF-beta and SMAD3driven induction of the epithelial-to-mesenchymal transition and migration of breast epithelial cells. Free Radic Biol Med 53: 1489-1499, 2012.

29. Brandes RP, Weissmann N, and Schroder K. Nox family NADPH oxidases: molecular mechanisms of activation. Free Radic Biol Med 76: 208-226, 2014.

30. Bravo-Cordero JJ, Magalhaes MA, Eddy RJ, Hodgson L, and Condeelis J. Functions of cofilin in cell locomotion and invasion. Nat Rev Mol Cell Biol 14: 405-415, 2013.

31. Brown DI and Griendling KK. Nox proteins in signal transduction. Free Radic Biol Med 47: 1239-1253, 2009. 
32. Brown NS, Jones A, Fujiyama C, Harris AL, and Bicknell R. Thymidine phosphorylase induces carcinoma cell oxidative stress and promotes secretion of angiogenic factors. Cancer Res 60: 6298-6302, 2000.

33. Cammarota F, de Vita G, Salvatore M, and Laukkanen MO. Ras oncogene-mediated progressive silencing of extracellular superoxide dismutase in tumorigenesis. Biomed Res Int 2015: 780409, 2015.

34. Cammarota F, Fiscardi F, Esposito T, de Vita G, Salvatore $\mathrm{M}$, and Laukkanen MO. Clinical relevance of thyroid cell models in redox research. Cancer Cell Int 15: 113, 2015.

35. Cammarota F and Laukkanen MO. Mesenchymal stem/ stromal cells in stromal evolution and cancer progression. Stem Cells Int 2016: 4824573, 2016.

36. Cantisani MC, Parascandolo A, Perala M, Allocca C, Fey V, Sahlberg N, Merolla F, Basolo F, Laukkanen MO, Kallioniemi OP, Santoro M, and Castellone MD. A lossof-function genetic screening identifies novel mediators of thyroid cancer cell viability. Oncotarget 7: 28510-28522, 2016.

37. Carini F, Mazzola M, Rappa F, Jurjus A, Geagea AG, Al Kattar S, Bou-Assi T, Jurjus R, Damiani P, Leone A, and Tomasello G. Colorectal carcinogenesis: role of oxidative stress and antioxidants. Anticancer Res 37: 4759-4766, 2017.

38. Carlsson LM, Marklund SL, and Edlund T. The rat extracellular superoxide dismutase dimer is converted to a tetramer by the exchange of a single amino acid. Proc Natl Acad Sci U S A 93: 5219-5222, 1996.

39. Castellone MD, Langella A, Cantara S, Laurila JP, Laatikainen LE, Bellelli R, Pacini F, Salvatore M, and Laukkanen MO. Extracellular superoxide dismutase induces mouse embryonic fibroblast proliferative burst, growth arrest, immortalization, and consequent in vivo tumorigenesis. Antioxid Redox Signal 21: 1460-1474, 2014.

40. Castellone MD and Laukkanen MO. TGF-beta1, WNT, and $\mathrm{SHH}$ signaling in tumor progression and in fibrotic diseases. Front Biosci (Schol Ed) 9: 31-45, 2017.

41. Castellone MD, Laukkanen MO, Teramoto H, Bellelli R, Ali G, Fontanini G, Santoro M, and Gutkind JS. Cross talk between the bombesin neuropeptide receptor and Sonic hedgehog pathways in small cell lung carcinoma. Oncogene 34: 1679-1687, 2015.

42. Castellone MD, Teramoto H, Williams BO, Druey KM, and Gutkind JS. Prostaglandin E2 promotes colon cancer cell growth through a Gs-axin-beta-catenin signaling axis. Science 310: 1504-1510, 2005.

43. Castellone MD, Verrienti A, Magendra Rao D, Sponziello M, Fabbro D, Muthu M, Durante C, Maranghi M, Damante G, Pizzolitto S, Costante G, Russo D, Santoro M, and Filetti S. A novel de novo germ-line V292M mutation in the extracellular region of RET in a patient with phaeochromocytoma and medullary thyroid carcinoma: functional characterization. Clin Endocrinol (Oxf) 73: 529-534, 2010.

44. Chan JY, Kwong M, Lo M, Emerson R, and Kuypers FA. Reduced oxidative-stress response in red blood cells from p45NFE2-deficient mice. Blood 97: 2151-2158, 2001.

45. Check J, Byrd CL, Menio J, Rippe RA, Hines IN, and Wheeler MD. Src kinase participates in LPS-induced activation of NADPH oxidase. Mol Immunol 47: 756-762, 2010.

46. Chen DS and Mellman I. Elements of cancer immunity and the cancer-immune set point. Nature 541: 321-330, 2017.
47. Chen F, Haigh S, Barman S, and Fulton DJ. From form to function: the role of Nox4 in the cardiovascular system. Front Physiol 3: 412, 2012.

48. Chen F, Yu Y, Haigh S, Johnson J, Lucas R, Stepp DW, and Fulton DJ. Regulation of NADPH oxidase 5 by protein kinase C isoforms. PloS One 9: e88405, 2014.

49. Chen P, Levis M, Brown P, Kim KT, Allebach J, and Small D. FLT3/ITD mutation signaling includes suppression of SHP-1. J Biol Chem 280: 5361-5369, 2005.

50. Cheng G, Cao Z, Xu X, van Meir EG, and Lambeth JD. Homologs of gp91phox: cloning and tissue expression of Nox3, Nox4, and Nox5. Gene 269: 131-140, 2001.

51. Cheng $G$ and Lambeth JD. NOXO1, regulation of lipid binding, localization, and activation of Nox 1 by the Phox homology (PX) domain. J Biol Chem 279: 4737-4742, 2004.

52. Cheng G, Ritsick D, and Lambeth JD. Nox3 regulation by NOXO1, p47phox, and p67phox. J Biol Chem 279: 34250-34255, 2004.

53. Chervenick PA and Boggs DR. Patterns of proliferation and differentiation of hematopoietic stem cells after compartment depletion. Blood 37: 568-580, 1971.

54. Cheung TH and Rando TA. Molecular regulation of stem cell quiescence. Nat Rev Mol Cell Biol 14: 329-340, 2013.

55. Choi JA, Jung YS, Kim JY, Kim HM, and Lim IK. Inhibition of breast cancer invasion by TIS21/BTG2/Pc3Akt1-Sp1-Nox4 pathway targeting actin nucleators, mDia genes. Oncogene 35: 83-93, 2016.

56. Chow DC, Wenning LA, Miller WM, and Papoutsakis ET. Modeling $\mathrm{pO}(2)$ distributions in the bone marrow hematopoietic compartment. I. Krogh's model. Biophys $J$ 81: 675-684, 2001.

57. Chowdhury AK, Watkins T, Parinandi NL, Saatian B, Kleinberg ME, Usatyuk PV, and Natarajan V. Srcmediated tyrosine phosphorylation of p47phox in hyperoxia-induced activation of NADPH oxidase and generation of reactive oxygen species in lung endothelial cells. J Biol Chem 280: 20700-20711, 2005.

58. Chung YW, Kim HK, Kim IY, Yim MB, and Chock PB. Dual function of protein kinase $\mathrm{C}$ (PKC) in 12-Otetradecanoylphorbol-13-acetate (TPA)-induced manganese superoxide dismutase (MnSOD) expression: activation of CREB and FOXO3a by PKC-alpha phosphorylation and by PKC-mediated inactivation of Akt, respectively. $J$ Biol Chem 286: 29681-29690, 2011.

59. Church SL, Grant JW, Ridnour LA, Oberley LW, Swanson PE, Meltzer PS, and Trent JM. Increased manganese superoxide dismutase expression suppresses the malignant phenotype of human melanoma cells. Proc Natl Acad Sci U S A 90: 3113-3117, 1993.

60. Collado R, Oliver I, Tormos C, Egea M, Miguel A, Cerda C, Ivars D, Borrego S, Carbonell F, and Saez GT. Early ROS-mediated DNA damage and oxidative stress biomarkers in Monoclonal B Lymphocytosis. Cancer Lett 317: 144-149, 2012.

61. Connor KM, Hempel N, Nelson KK, Dabiri G, Gamarra A, Belarmino J, Van De Water L, Mian BM, and Melendez JA. Manganese superoxide dismutase enhances the invasive and migratory activity of tumor cells. Cancer Res 67: 10260-10267, 2007.

62. Connor KM, Subbaram S, Regan KJ, Nelson KK, Mazurkiewicz JE, Bartholomew PJ, Aplin AE, Tai YT, Aguirre-Ghiso J, Flores SC, and Melendez JA. Mitochondrial $\mathrm{H}_{2} \mathrm{O}_{2}$ regulates the angiogenic phenotype via PTEN oxidation. J Biol Chem 280: 16916-16924, 2005. 
63. Cox BD, Natarajan M, Stettner MR, and Gladson CL. New concepts regarding focal adhesion kinase promotion of cell migration and proliferation. J Cell Biochem 99: 3552, 2006.

64. Cross AR and Segal AW. The NADPH oxidase of professional phagocytes-prototype of the NOX electron transport chain systems. Biochim Biophys Acta 1657: 122, 2004.

65. Cucoranu I, Clempus R, Dikalova A, Phelan PJ, Ariyan S, Dikalov S, and Sorescu D. NAD(P)H oxidase 4 mediates transforming growth factor-betal-induced differentiation of cardiac fibroblasts into myofibroblasts. Circ Res 97: 900-907, 2005.

66. Cuevas-Ramos G, Petit CR, Marcq I, Boury M, Oswald E, and Nougayrede JP. Escherichia coli induces DNA damage in vivo and triggers genomic instability in mammalian cells. Proc Natl Acad Sci U S A 107: 11537-11542, 2010.

67. Cui Y, She K, Tian D, Zhang P, and Xin X. miR-146a inhibits proliferation and enhances chemosensitivity in epithelial ovarian cancer via reduction of SOD2. Oncol Res 23: 275-282, 2016.

68. Dalmonte ME, Forte E, Genova ML, Giuffre A, Sarti P, and Lenaz G. Control of respiration by cytochrome $\mathrm{c}$ oxidase in intact cells: role of the membrane potential. $J$ Biol Chem 284: 32331-32335, 2009.

69. Damiano S, Petrozziello T, Ucci V, Amente S, Santillo M, and Mondola P. Cu-Zn superoxide dismutase activates muscarinic acetylcholine M1 receptor pathway in neuroblastoma cells. Mol Cell Neurosci 52: 31-37, 2013.

70. de Groot RE, Rappel SB, Lorenowicz MJ, and Korswagen HC. Protein kinase CK2 is required for Wntless internalization and Wnt secretion. Cell Signal 26: 2601-2605, 2014.

71. de Jong PR, Taniguchi K, Harris AR, Bertin S, Takahashi N, Duong J, Campos AD, Powis G, Corr M, Karin M, and Raz E. ERK5 signalling rescues intestinal epithelial turnover and tumour cell proliferation upon ERK1/2 abrogation. Nat Commun 7: 11551, 2016.

72. Debbabi M, Kroviarski Y, Bournier O, Gougerot-Pocidalo MA, El-Benna J, and Dang PM. NOXO1 phosphorylation on serine 154 is critical for optimal NADPH oxidase 1 assembly and activation. FASEB $J$ 27: 1733-1748, 2013.

73. DerMardirossian $C$ and Bokoch GM. GDIs: central regulatory molecules in Rho GTPase activation. Trends Cell Biol 15: 356-363, 2005.

74. Diaz B, Shani G, Pass I, Anderson D, Quintavalle M, and Courtneidge SA. Tks5-dependent, nox-mediated generation of reactive oxygen species is necessary for invadopodia formation. Sci Signal 2: ra53, 2009.

75. Diebold BA and Bokoch GM. Molecular basis for Rac2 regulation of phagocyte NADPH oxidase. Nat Immunol 2: 211-215, 2001.

76. Dolado I, Swat A, Ajenjo N, De Vita G, Cuadrado A, and Nebreda AR. P38alpha MAP kinase as a sensor of reactive oxygen species in tumorigenesis. Cancer Cell 11: 191205, 2007.

77. Dominici M, Rasini V, Bussolari R, Chen X, Hofmann TJ, Spano C, Bernabei D, Veronesi E, Bertoni F, Paolucci P, Conte P, and Horwitz EM. Restoration and reversible expansion of the osteoblastic hematopoietic stem cell niche after marrow radioablation. Blood 114: 2333-2343, 2009.

78. Donaldson K, Stone V, Borm PJ, Jimenez LA, Gilmour PS, Schins RP, Knaapen AM, Rahman I, Faux SP, Brown $\mathrm{DM}$, and MacNee W. Oxidative stress and calcium sig- naling in the adverse effects of environmental particles (PM10). Free Radic Biol Med 34: 1369-1382, 2003.

79. Dong H, Strome SE, Salomao DR, Tamura H, Hirano F, Flies DB, Roche PC, Lu J, Zhu G, Tamada K, Lennon VA, Celis E, and Chen L. Tumor-associated B7-H1 promotes T-cell apoptosis: a potential mechanism of immune evasion. Nat Med 8: 793-800, 2002.

80. Doppler $\mathrm{H}$ and Storz P. Mitochondrial and oxidative stress-mediated activation of protein kinase D1 and its importance in pancreatic cancer. Front Oncol 7: 41, 2017.

81. Dorsam RT and Gutkind JS. G-protein-coupled receptors and cancer. Nat Rev Cancer 7: 79-94, 2007.

82. Duan J, Duan J, Zhang Z, and Tong T. Irreversible cellular senescence induced by prolonged exposure to $\mathrm{H}_{2} \mathrm{O}_{2}$ involves DNA-damage-and-repair genes and telomere shortening. Int J Biochem Cell Biol 37: 1407-1420, 2005.

83. Duffy MJ, Maguire TM, Hill A, McDermott E, and O'Higgins N. Metalloproteinases: role in breast carcinogenesis, invasion and metastasis. Breast Cancer Res 2: 252-257, 2000.

84. Ekoue DN, He C, Diamond AM, and Bonini MG. Manganese superoxide dismutase and glutathione peroxidase-1 contribute to the rise and fall of mitochondrial reactive oxygen species which drive oncogenesis. Biochim Biophys Acta 1858: 628-632, 2017.

85. el Benna J, Faust LP, and Babior BM. The phosphorylation of the respiratory burst oxidase component $\mathrm{p} 47 \mathrm{phox}$ during neutrophil activation. Phosphorylation of sites recognized by protein kinase $\mathrm{C}$ and by proline-directed kinases. J Biol Chem 269: 23431-23436, 1994.

86. el Benna J, Ruedi JM, and Babior BM. Cytosolic guanine nucleotide-binding protein Rac2 operates in vivo as a component of the neutrophil respiratory burst oxidase. Transfer of Rac2 and the cytosolic oxidase components p47phox and p67phox to the submembranous actin cytoskeleton during oxidase activation. J Biol Chem 269: 6729-6734, 1994.

87. El Jamali A, Valente AJ, Lechleiter JD, Gamez MJ, Pearson DW, Nauseef WM, and Clark RA. Novel redoxdependent regulation of NOX5 by the tyrosine kinase cAbl. Free Radic Biol Med 44: 868-881, 2008.

88. Ellison MA, Thurman G, Gearheart CM, Seewald RH, Porter CC, and Ambruso DR. INF-gamma enhances Nox2 activity by upregulating phox proteins when applied to differentiating PLB-985 cells but does not induce Nox2 activity by itself. PloS One 10: e0136766, 2015.

89. Eun HS, Cho SY, Joo JS, Kang SH, Moon HS, Lee ES, $\mathrm{Kim} \mathrm{SH}$, and Lee BS. Gene expression of NOX family members and their clinical significance in hepatocellular carcinoma. Sci Rep 7: 11060, 2017.

90. Finkel T, Serrano M, and Blasco MA. The common biology of cancer and ageing. Nature 448: 767-774, 2007.

91. Folz RJ, Abushamaa AM, and Suliman HB. Extracellular superoxide dismutase in the airways of transgenic mice reduces inflammation and attenuates lung toxicity following hyperoxia. J Clin Invest 103: 10551066, 1999.

92. Fontayne A, Dang PM, Gougerot-Pocidalo MA, and ElBenna J. Phosphorylation of p47phox sites by PKC alpha, beta II, delta, and zeta: effect on binding to p22phox and on NADPH oxidase activation. Biochemistry 41: 77437750, 2002.

93. Forster D and Reiser G. Nucleotides protect rat brain astrocytes against hydrogen peroxide toxicity and induce 
antioxidant defense via $\mathrm{P} 2 \mathrm{Y}$ receptors. Neurochem Int 94: 57-66, 2016.

94. Fu X, Beer DG, Behar J, Wands J, Lambeth D, and Cao $\mathrm{W}$. cAMP-response element-binding protein mediates acid-induced NADPH oxidase NOX5-S expression in Barrett esophageal adenocarcinoma cells. J Biol Chem 281: 20368-20382, 2006.

95. Fuchs A, Dagher MC, Faure J, and Vignais PV. Topological organization of the cytosolic activating complex of the superoxide-generating NADPH-oxidase. Pinpointing the sites of interaction between $\mathrm{p} 47 \mathrm{phoz}, \mathrm{p} 67$ phox and p40phox using the two-hybrid system. Biochim Biophys Acta 1312: 39-47, 1996.

96. Fulton DJ. Nox 5 and the regulation of cellular function. Antioxid Redox Signal 11: 2443-2452, 2009.

97. Gan J, Meng F, Zhou X, Li C, He Y, Zeng X, Jiang X, Liu J, Zeng G, Tang Y, Liu M, Mrsny RJ, Hu X, Hu J, and Li $\mathrm{T}$. Hematopoietic recovery of acute radiation syndrome by human superoxide dismutase-expressing umbilical cord mesenchymal stromal cells. Cytotherapy 17: 403-417, 2015.

98. Garinis GA, van der Horst GT, Vijg J, and Hoeijmakers JH. DNA damage and ageing: new-age ideas for an ageold problem. Nat Cell Biol 10: 1241-1247, 2008.

99. Gatz SA and Wiesmuller L. p53 in recombination and repair. Cell Death Differ 13: 1003-1016, 2006.

100. Gazdar AF, Bunn PA, and Minna JD. Small-cell lung cancer: what we know, what we need to know and the path forward. Nat Rev Cancer 17: 725-737, 2017.

101. Geiszt M, Lekstrom K, Brenner S, Hewitt SM, Dana R, Malech HL, and Leto TL. NAD(P)H oxidase 1, a product of differentiated colon epithelial cells, can partially replace glycoprotein 91 phox in the regulated production of superoxide by phagocytes. J Immunol 171: 299-306, 2003.

102. Geiszt M, Lekstrom K, Witta J, and Leto TL. Proteins homologous to $\mathrm{p} 47 \mathrm{phox}$ and $\mathrm{p} 67$ phox support superoxide production by $\mathrm{NAD}(\mathrm{P}) \mathrm{H}$ oxidase 1 in colon epithelial cells. J Biol Chem 278: 20006-20012, 2003.

103. Getzoff ED, Tainer JA, Weiner PK, Kollman PA, Richardson JS, and Richardson DC. Electrostatic recognition between superoxide and copper, zinc superoxide dismutase. Nature 306: 287-290, 1983.

104. Ghossein R. Update to the College of American Pathologists reporting on thyroid carcinomas. Head Neck Pathol 3: 86-93, 2009.

105. Gianni D, Bohl B, Courtneidge SA, and Bokoch GM. The involvement of the tyrosine kinase $\mathrm{c}-\mathrm{Src}$ in the regulation of reactive oxygen species generation mediated by NADPH oxidase-1. Mol Biol Cell 19: 2984-2994, 2008.

106. Gianni D, DerMardirossian C, and Bokoch GM. Direct interaction between Tks proteins and the N-terminal proline-rich region (PRR) of NoxA1 mediates Nox1dependent ROS generation. Eur J Cell Biol 90: 164-171, 2011.

107. Gianni D, Diaz B, Taulet N, Fowler B, Courtneidge SA, and Bokoch GM. Novel p47(phox)-related organizers regulate localized NADPH oxidase 1 (Nox1) activity. Sci Signal 2: ra54, 2009.

108. Ginis I and Tauber AI. Activation mechanisms of adherent human neutrophils. Blood 76: 1233-1239, 1990.

109. Giordano TJ, Au AY, Kuick R, Thomas DG, Rhodes DR, Wilhelm KG, Jr., Vinco M, Misek DE, Sanders D, Zhu Z, Ciampi R, Hanash S, Chinnaiyan A, Clifton-Bligh RJ, Robinson BG, Nikiforov YE, and Koenig RJ. Delineation, functional validation, and bioinformatic evaluation of gene expression in thyroid follicular carcinomas with the PAX8-PPARG translocation. Clin Cancer Res 12: 19831993, 2006.

110. Glasauer A, Sena LA, Diebold LP, Mazar AP, and Chandel NS. Targeting SOD1 reduces experimental nonsmall-cell lung cancer. J Clin Invest 124: 117-128, 2014.

111. Godfrey R, Arora D, Bauer R, Stopp S, Muller JP, Heinrich T, Bohmer SA, Dagnell M, Schnetzke U, Scholl S, Ostman A, and Bohmer FD. Cell transformation by FLT3 ITD in acute myeloid leukemia involves oxidative inactivation of the tumor suppressor protein-tyrosine phosphatase DEP-1/PTPRJ. Blood 119: 4499-4511, 2012.

112. Gorlach A, Bertram K, Hudecova S, and Krizanova O. Calcium and ROS: a mutual interplay. Redox Biol 6: 260 271, 2015.

113. Graham KA, Kulawiec M, Owens KM, Li X, Desouki MM, Chandra D, and Singh KK. NADPH oxidase 4 is an oncoprotein localized to mitochondria. Cancer Biol Ther 10: 223-231, 2010.

114. Gregg JL, Turner RM, 2nd, Chang G, Joshi D, Zhan Y, Chen L, and Maranchie JK. NADPH oxidase NOX4 supports renal tumorigenesis by promoting the expression and nuclear accumulation of HIF2alpha. Cancer Res 74: 3501-3511, 2014.

115. Gryfe R. Inherited colorectal cancer syndromes. Clin Colon Rectal Surg 22: 198-208, 2009.

116. Haklar G, Sayin-Ozveri E, Yuksel M, Aktan AO, and Yalcin AS. Different kinds of reactive oxygen and nitrogen species were detected in colon and breast tumors. Cancer Lett 165: 219-224, 2001.

117. Hamdi Y, Kaddour H, Vaudry D, Douiri S, Bahdoudi S, Leprince J, Castel H, Vaudry H, Amri M, Tonon MC, and Masmoudi-Kouki O. The stimulatory effect of the octadecaneuropeptide ODN on astroglial antioxidant enzyme systems is mediated through a GPCR. Front Endocrinol (Lausanne) 3: 138, 2012.

118. Haniu AE, Maricato JT, Mathias PP, Castilho DG, Miguel RB, Monteiro HP, Puccia R, and Batista WL. Low concentrations of hydrogen peroxide or nitrite induced of Paracoccidioides brasiliensis cell proliferation in a Rasdependent manner. PloS One 8: e69590, 2013.

119. Hanley CJ, Mellone M, Ford K, Thirdborough SM, Mellows T, Frampton SJ, Smith DM, Harden E, Szyndralewiez C, Bullock M, Noble F, Moutasim KA, King EV, Vijayanand P, Mirnezami AH, Underwood TJ, Ottensmeier $\mathrm{CH}$, and Thomas GJ. Targeting the myofibroblastic cancer-associated fibroblast phenotype through inhibition of NOX4. J Natl Cancer Inst 110: 1, 2018.

120. Harraz MM, Marden JJ, Zhou W, Zhang Y, Williams A, Sharov VS, Nelson K, Luo M, Paulson H, Schoneich C, and Engelhardt JF. SOD1 mutations disrupt redoxsensitive Rac regulation of NADPH oxidase in a familial ALS model. J Clin Invest 118: 659-670, 2008.

121. Hart PC, Mao M, de Abreu AL, Ansenberger-Fricano K, Ekoue DN, Ganini D, Kajdacsy-Balla A, Diamond AM, Minshall RD, Consolaro ME, Santos JH, and Bonini MG. MnSOD upregulation sustains the Warburg effect via mitochondrial ROS and AMPK-dependent signalling in cancer. Nat Commun 6: 6053, 2015.

122. Hartung HP, Parnham MJ, Winkelmann J, Englberger W, and Hadding U. Platelet activating factor (PAF) induces the oxidative burst in macrophages. Int J Immunopharmacol 5: 115-121, 1983. 
123. Hawkins BJ, Solt LA, Chowdhury I, Kazi AS, Abid MR, Aird WC, May MJ, Foskett JK, and Madesh M. G proteincoupled receptor $\mathrm{Ca}^{2+}$-linked mitochondrial reactive oxygen species are essential for endothelial/leukocyte adherence. Mol Cell Biol 27: 7582-7593, 2007.

124. He H, Jazdzewski K, Li W, Liyanarachchi S, Nagy R, Volinia S, Calin GA, Liu CG, Franssila K, Suster S, Kloos RT, Croce CM, and de la Chapelle A. The role of microRNA genes in papillary thyroid carcinoma. Proc Natl Acad Sci U S A 102: 19075-19080, 2005.

125. Hemachandra LP, Shin DH, Dier U, Iuliano JN, Engelberth SA, Uusitalo LM, Murphy SK, and Hempel N. Mitochondrial superoxide dismutase has a protumorigenic role in ovarian clear cell carcinoma. Cancer Res 75: 4973-4984, 2015.

126. Hempel N, Carrico PM, and Melendez JA. Manganese superoxide dismutase (SOD2) and redox-control of signaling events that drive metastasis. Anticancer Agents Med Chem 11: 191-201, 2011.

127. Hempel N and Melendez JA. Intracellular redox status controls membrane localization of pro- and anti-migratory signaling molecules. Redox Biol 2: 245-250, 2014.

128. Hempel N, and Trebak M. Crosstalk between calcium and reactive oxygen species signaling in cancer. Cell Calcium 63: 70-96, 2017.

129. Hempel N, Ye H, Abessi B, Mian B, and Melendez JA. Altered redox status accompanies progression to metastatic human bladder cancer. Free Radic Biol Med 46: 4250, 2009.

130. Heyworth PG, Knaus UG, Xu X, Uhlinger DJ, Conroy L, Bokoch GM, and Curnutte JT. Requirement for posttranslational processing of Rac GTP-binding proteins for activation of human neutrophil NADPH oxidase. Mol Biol Cell 4: 261-269, 1993.

131. Hitchler MJ and Domann FE. Regulation of CuZnSOD and its redox signaling potential: implications for amyotrophic lateral sclerosis. Antioxid Redox Signal 20: 15901598, 2014.

132. Hoeijmakers JH. DNA damage, aging, and cancer. $N$ Engl J Med 361: 1475-1485, 2009.

133. Hoffman M and Autor AP. Production of superoxide anion by an NADPH-oxidase from rat pulmonary macrophages. FEBS Lett 121: 352-354, 1980.

134. Holdfeldt A, Dahlstrand Rudin A, Gabl M, Rajabkhani Z, Konig GM, Kostenis E, Dahlgren C, and Forsman H. Reactivation of Galphai-coupled formyl peptide receptors is inhibited by Galphaq-selective inhibitors when induced by signals generated by the platelet-activating factor receptor. J Leukoc Biol 102: 871-880, 2017.

135. Hole PS, Zabkiewicz J, Munje C, Newton Z, Pearn L, White P, Marquez N, Hills RK, Burnett AK, Tonks A, and Darley RL. Overproduction of NOX-derived ROS in AML promotes proliferation and is associated with defective oxidative stress signaling. Blood 122: 3322-3330, 2013.

136. Holl M, Koziel R, Schafer G, Pircher H, Pauck A, Hermann M, Klocker H, Jansen-Durr P, and Sampson N. ROS signaling by NADPH oxidase 5 modulates the proliferation and survival of prostate carcinoma cells. Mol Carcinog 55: 27-39, 2016.

137. Holz GG and Chepurny OG. Diabetes outfoxed by GLP1? Sci STKE 2005: pe2, 2005.

138. Hong J, Behar J, Wands J, Resnick M, Wang LJ, Delellis RA, Lambeth D, and Cao W. Bile acid reflux contributes to development of esophageal adenocarcinoma via activation of phosphatidylinositol-specific phospholipase Cgamma2 and NADPH oxidase NOX5-S. Cancer Res 70: 1247-1255, 2010.

139. Hong J, Li D, and Cao W. Rho kinase ROCK2 mediates acid-induced NADPH oxidase NOX5-S expression in human esophageal adenocarcinoma cells. PloS One 11: e0149735, 2016.

140. Hoyal CR, Gutierrez A, Young BM, Catz SD, Lin JH, Tsichlis PN, and Babior BM. Modulation of p47PHOX activity by site-specific phosphorylation: Akt-dependent activation of the NADPH oxidase. Proc Natl Acad Sci U S A 100: 5130-5135, 2003.

141. Hu L, Cheng H, Gao Y, Shi M, Liu Y, Hu Z, Xu J, Qiu L, Yuan W, Leung AY, Yang YG, and Cheng T. Antioxidant $\mathrm{N}$-acetyl-L-cysteine increases engraftment of human hematopoietic stem cells in immune-deficient mice. Blood 124: e45-e48, 2014.

142. Huang P, Feng L, Oldham EA, Keating MJ, and Plunkett W. Superoxide dismutase as a target for the selective killing of cancer cells. Nature 407: 390-395, 2000.

143. Hughes R, Qian BZ, Rowan C, Muthana M, Keklikoglou I, Olson OC, Tazzyman S, Danson S, Addison C, Clemons M, Gonzalez-Angulo AM, Joyce JA, De Palma M, Pollard JW, and Lewis CE. Perivascular M2 macrophages stimulate tumor relapse after chemotherapy. Cancer Res 75: 3479-3491, 2015.

144. Imamura K, Izumi Y, Watanabe A, Tsukita K, Woltjen K, Yamamoto T, Hotta A, Kondo T, Kitaoka S, Ohta A, Tanaka A, Watanabe D, Morita M, Takuma H, Tamaoka A, Kunath T, Wray S, Furuya H, Era T, Makioka K, Okamoto K, Fujisawa T, Nishitoh H, Homma K, Ichijo H, Julien JP, Obata N, Hosokawa M, Akiyama H, Kaneko S, Ayaki T, Ito H, Kaji R, Takahashi R, Yamanaka S, and Inoue $\mathrm{H}$. The $\mathrm{Src} / \mathrm{c}-\mathrm{Abl}$ pathway is a potential therapeutic target in amyotrophic lateral sclerosis. Sci Transl Med 9: pii: eaaf3962, 2017.

145. Isern J, He Z, Fraser ST, Nowotschin S, Ferrer-Vaquer A, Moore R, Hadjantonakis AK, Schulz V, Tuck D, Gallagher PG, and Baron MH. Single-lineage transcriptome analysis reveals key regulatory pathways in primitive erythroid progenitors in the mouse embryo. Blood 117: 4924-4934, 2011.

146. Ishida T, Suzuki S, Lai CY, Yamazaki S, Kakuta S, Iwakura Y, Nojima M, Takeuchi Y, Higashihara M, Nakauchi $\mathrm{H}$, and Otsu M. Pre-Transplantation blockade of TNF-alpha-mediated oxygen species accumulation protects hematopoietic stem cells. Stem Cells 35: 989-1002, 2017.

147. Itkin T, Gur-Cohen S, Spencer JA, Schajnovitz A, Ramasamy SK, Kusumbe AP, Ledergor G, Jung Y, Milo I, Poulos MG, Kalinkovich A, Ludin A, Kollet O, Shakhar G, Butler JM, Rafii S, Adams RH, Scadden DT, Lin CP, and Lapidot T. Distinct bone marrow blood vessels differentially regulate haematopoiesis. Nature 532: 323-328, 2016.

148. Ito K, Hirao A, Arai F, Matsuoka S, Takubo K, Hamaguchi I, Nomiyama K, Hosokawa K, Sakurada K, Nakagata N, Ikeda Y, Mak TW, and Suda T. Regulation of oxidative stress by ATM is required for self-renewal of haematopoietic stem cells. Nature 431: 997-1002, 2004.

149. Ito K, Hirao A, Arai F, Takubo K, Matsuoka S, Miyamoto K, Ohmura M, Naka K, Hosokawa K, Ikeda Y, and Suda T. Reactive oxygen species act through p38 MAPK to 
limit the lifespan of hematopoietic stem cells. Nat Med 12: 446-451, 2006.

150. Jaffe $A B$ and Hall A. Rho GTPases: biochemistry and biology. Annu Rev Cell Dev Biol 21: 247-269, 2005.

151. Jan M, Snyder TM, Corces-Zimmerman MR, Vyas P, Weissman IL, Quake SR, and Majeti R. Clonal evolution of preleukemic hematopoietic stem cells precedes human acute myeloid leukemia. Sci Transl Med 4: 149ra118, 2012.

152. Jana A, Krett NL, Guzman G, Khalid A, Ozden O, Staudacher JJ, Bauer J, Baik SH, Carroll T, Yazici C, and Jung B. $\mathrm{NF} \kappa \mathrm{B}$ is essential for activin-induced colorectal cancer migration via upregulation of PI3K-MDM2 pathway. Oncotarget 8: 37377-37393, 2017.

153. Jang YY and Sharkis SJ. A low level of reactive oxygen species selects for primitive hematopoietic stem cells that may reside in the low-oxygenic niche. Blood 110: 30563063, 2007.

154. Jay DB, Papaharalambus CA, Seidel-Rogol B, Dikalova AE, Lassegue B, and Griendling KK. Nox5 mediates PDGF-induced proliferation in human aortic smooth muscle cells. Free Radic Biol Med 45: 329-335, 2008.

155. Jayavelu AK, Moloney JN, Bohmer FD, and Cotter TG. NOX-driven ROS formation in cell transformation of FLT3-ITD-positive AML. Exp Hematol 44: 1113-1122, 2016.

156. Jayavelu AK, Muller JP, Bauer R, Bohmer SA, Lassig J, Cerny-Reiterer S, Sperr WR, Valent P, Maurer B, Moriggl R, Schroder K, Shah AM, Fischer M, Scholl S, Barth J, Oellerich T, Berg T, Serve H, Frey S, Fischer T, Heidel FH, and Bohmer FD. NOX4-driven ROS formation mediates PTP inactivation and cell transformation in FLT3ITDpositive AML cells. Leukemia 30: 473-483, 2016.

157. Johnson F and Giulivi C. Superoxide dismutases and their impact upon human health. Mol Aspects Med 26: 340 352, 2005.

158. Juarez JC, Manuia M, Burnett ME, Betancourt O, Boivin B, Shaw DE, Tonks NK, Mazar AP, and Donate F. Superoxide dismutase 1 (SOD1) is essential for $\mathrm{H}_{2} \mathrm{O}_{2}$ mediated oxidation and inactivation of phosphatases in growth factor signaling. Proc Natl Acad Sci U S A 105: 7147-7152, 2008.

159. Juhasz A, Ge Y, Markel S, Chiu A, Matsumoto L, van Balgooy J, Roy K, and Doroshow JH. Expression of NADPH oxidase homologues and accessory genes in human cancer cell lines, tumours and adjacent normal tissues. Free Radic Res 43: 523-532, 2009.

160. Kadenbach B and Huttemann M. The subunit composition and function of mammalian cytochrome c oxidase. $\mathrm{Mi}$ tochondrion 24: 64-76, 2015.

161. Kamarajugadda S, Cai Q, Chen H, Nayak S, Zhu J, He M, Jin Y, Zhang Y, Ai L, Martin SS, Tan M, and Lu J. Manganese superoxide dismutase promotes anoikis resistance and tumor metastasis. Cell Death Dis 4: e504, 2013.

162. Karlsson K and Marklund SL. Heparin-induced release of extracellular superoxide dismutase to human blood plasma. Biochem J 242: 55-59, 1987.

163. Karnati S, Luers G, Pfreimer S, and Baumgart-Vogt E. Mammalian SOD2 is exclusively located in mitochondria and not present in peroxisomes. Histochem Cell Biol 140: 105-117, 2013.

164. Kato M, Marumo M, Nakayama J, Matsumoto M, YabeNishimura C, and Kamata T. The ROS-generating oxidase Nox 1 is required for epithelial restitution following colitis. Exp Anim 65: 197-205, 2016.
165. Kenny TC, Hart P, Ragazzi M, Sersinghe M, Chipuk J, Sagar MA, Eliceiri KW, LaFramboise T, Grandhi S, Santos J, Riar AK, Papa L, D'Aurello M, Manfredi G, Bonini MG, and Germain D. Selected mitochondrial DNA landscapes activate the SIRT3 axis of the UPRmt to promote metastasis. Oncogene 36: 4393-4404, 2017.

166. Kero J, Ahmed K, Wettschureck N, Tunaru S, Wintermantel T, Greiner E, Schutz G, and Offermanns S. Thyrocyte-specific Gq/G11 deficiency impairs thyroid function and prevents goiter development. J Clin Invest 117: 2399-2407, 2007.

167. Ki DH, Jeung HC, Park CH, Kang SH, Lee GY, Lee WS, Kim NK, Chung HC, and Rha SY. Whole genome analysis for liver metastasis gene signatures in colorectal cancer. Int J Cancer 121: 2005-2012, 2007.

168. Kigawa Y, Miyazaki T, Lei XF, Kim-Kaneyama JR, and Miyazaki A. Functional heterogeneity of Nadph oxidases in atherosclerotic and aneurysmal diseases. $J$ Atheroscler Thromb 24: 1-13, 2017.

169. Kim CG, Lee JJ, Jung DY, Jeon J, Heo HS, Kang HC, Shin JH, Cho YS, Cha KJ, Kim CG, Do BR, Kim KS, and Kim HS. Profiling of differentially expressed genes in human stem cells by cDNA microarray. Mol Cells 21: 343-355, 2006.

170. Kim CH, Han SI, Lee SY, Youk HS, Moon JY, Duong HQ, Park MJ, Joo YM, Park HG, Kim YJ, Yoo MA, Lim $\mathrm{SC}$, and Kang HS. Protein kinase C-ERK1/2 signal pathway switches glucose depletion-induced necrosis to apoptosis by regulating superoxide dismutases and suppressing reactive oxygen species production in A549 lung cancer cells. J Cell Physiol 211: 371-385, 2007.

171. Kim H, Sung JY, Park EK, Kho S, Koo KH, Park SY, Goh $\mathrm{SH}$, Jeon YK, Oh S, Park BK, Jung YK, and Kim YN. Regulation of anoikis resistance by NADPH oxidase 4 and epidermal growth factor receptor. Br J Cancer 116: 370381, 2017.

172. Kim HT, Kim YH, Nam JW, Lee HJ, Rho HM, and Jung $\mathrm{G}$. Study of $5^{\prime}$-flanking region of human $\mathrm{Cu} / \mathrm{Zn}$ superoxide dismutase. Biochem Biophys Res Commun 201: 15261533, 1994.

173. Kim YN, Koo KH, Sung JY, Yun UJ, and Kim H. Anoikis resistance: an essential prerequisite for tumor metastasis. Int J Cell Biol 2012: 306879, 2012.

174. Kinsella S, Konig HG, and Prehn JH. Bid promotes K63linked polyubiquitination of tumor necrosis factor receptor associated factor 6 (TRAF6) and sensitizes to mutant SOD1-induced proinflammatory signaling in microglia. eNeuro 3: pii: ENEURO.0099-15.2016, 2016.

175. Kinugasa H, Whelan KA, Tanaka K, Natsuizaka M, Long A, Guo A, Chang S, Kagawa S, Srinivasan S, Guha M, Yamamoto K, St Clair DK, Avadhani NG, Diehl JA, and Nakagawa H. Mitochondrial SOD2 regulates epithelialmesenchymal transition and cell populations defined by differential CD44 expression. Oncogene 34: 5229-5239, 2015.

176. Koga $H$, Terasawa $H$, Nunoi $H$, Takeshige K, Inagaki F, and Sumimoto H. Tetratricopeptide repeat (TPR) motifs of p67(phox) participate in interaction with the small GTPase Rac and activation of the phagocyte NADPH oxidase. J Biol Chem 274: 25051-25060, 1999.

177. Koperek O, Asari R, Niederle B, and Kaserer K. Desmoplastic stromal reaction in papillary thyroid microcarcinoma. Histopathology 58: 919-924, 2011.

178. Koperek O, Scheuba C, Puri C, Birner P, Haslinger C, Rettig W, Niederle B, Kaserer K, and Garin Chesa P. 
Molecular characterization of the desmoplastic tumor stroma in medullary thyroid carcinoma. Int J Oncol 31: 59-67, 2007.

179. Kovacs CJ, Evans MJ, and Daly BM. A hematopoietic stromal lesion associated with fractionated radiotherapy (FxRT): time- and dose-effects. Anticancer Res 25: 28012807, 2005.

180. Krausova M and Korinek V. Wnt signaling in adult intestinal stem cells and cancer. Cell Signal 26: 570-579, 2014

181. Krijnen PA, Meischl C, Hack CE, Meijer CJ, Visser CA, Roos D, and Niessen HW. Increased Nox2 expression in human cardiomyocytes after acute myocardial infarction. J Clin Pathol 56: 194-199, 2003.

182. Kroviarski Y, Debbabi M, Bachoual R, Perianin A, Gougerot-Pocidalo MA, El-Benna J, and Dang PM. Phosphorylation of NADPH oxidase activator 1 (NOXA1) on serine 282 by MAP kinases and on serine 172 by protein kinase $\mathrm{C}$ and protein kinase A prevents NOX1 hyperactivation. FASEB $J$ 24: 2077-2092, 2010.

183. Kuwano Y, Kawahara T, Yamamoto H, Teshima-Kondo S, Tominaga K, Masuda K, Kishi K, Morita K, and Rokutan K. Interferon-gamma activates transcription of NADPH oxidase 1 gene and upregulates production of superoxide anion by human large intestinal epithelial cells. Am J Physiol Cell Physiol 290: C433-C443, 2006.

184. Laatikainen LE, Castellone MD, Hebrant A, Hoste C, Cantisani MC, Laurila JP, Salvatore G, Salerno P, Basolo F, Nasman J, Dumont JE, Santoro M, and Laukkanen MO. Extracellular superoxide dismutase is a thyroid differentiation marker down-regulated in cancer. Endocr Relat Cancer 17: 785-796, 2010.

185. Laatikainen LE, Incoronato M, Castellone MD, Laurila JP, Santoro M, and Laukkanen MO. SOD3 decreases ischemic injury derived apoptosis through phosphorylation of Erk1/2, Akt, and FoxO3a. PloS One 6: e24456, 2011.

186. Landi MT, Dracheva T, Rotunno M, Figueroa JD, Liu H, Dasgupta A, Mann FE, Fukuoka J, Hames M, Bergen AW, Murphy SE, Yang P, Pesatori AC, Consonni D, Bertazzi PA, Wacholder S, Shih JH, Caporaso NE, and Jen J. Gene expression signature of cigarette smoking and its role in lung adenocarcinoma development and survival. PloS One 3: e1651, 2008.

187. Lapidot $\mathrm{T}$ and Kollet $\mathrm{O}$. The essential roles of the chemokine SDF-1 and its receptor CXCR4 in human stem cell homing and repopulation of transplanted immunedeficient NOD/SCID and NOD/SCID/B2m(null) mice. Leukemia 16: 1992-2003, 2002.

188. Lassegue B, Sorescu D, Szocs K, Yin Q, Akers M, Zhang Y, Grant SL, Lambeth JD, and Griendling KK. Novel gp91(phox) homologues in vascular smooth muscle cells: nox 1 mediates angiotensin II-induced superoxide formation and redox-sensitive signaling pathways. Circ Res 88: 888-894, 2001.

189. Laukkanen MO. Extracellular superoxide dismutase: growth promoter or tumor suppressor? Oxid Med Cell Longev 2016: 3612589, 2016.

190. Laukkanen MO, Cammarota F, Esposito T, Salvatore M, and Castellone MD. Extracellular superoxide dismutase regulates the expression of small GTPase regulatory proteins GEFs, GAPs, and GDI. PloS One 10: e0121441, 2015.

191. Laukkanen MO and Castellone MD. Gastrin-releasing peptide receptor targeting in cancer treatment: emerging signaling networks and therapeutic applications. Curr Drug Targets 17: 508-514, 2016.
192. Laukkanen MO, Kivela A, Rissanen T, Rutanen J, Karkkainen MK, Leppanen O, Brasen JH, and Yla-Herttuala S. Adenovirus-mediated extracellular superoxide dismutase gene therapy reduces neointima formation in balloondenuded rabbit aorta. Circulation 106: 1999-2003, 2002.

193. Laukkanen MO, Kuramoto K, Calmels B, Takatoku M, von Kalle C, Donahue RE, and Dunbar CE. Low-dose total body irradiation causes clonal fluctuation of primate hematopoietic stem and progenitor cells. Blood 105: 1010-1015, 2005.

194. Laukkanen MO, Leppanen P, Turunen P, PorkkalaSarataho E, Salonen JT, and Yla-Herttuala S. Gene transfer of extracellular superoxide dismutase to atherosclerotic mice. Antioxid Redox Signal 3: 397-402, 2001.

195. Laukkanen MO, Leppanen P, Turunen P, Tuomisto T, Naarala J, and Yla-Herttuala S. EC-SOD gene therapy reduces paracetamol-induced liver damage in mice. $J$ Gene Med 3: 321-325, 2001.

196. Laurent E, McCoy JW, 3rd, Macina RA, Liu W, Cheng G, Robine S, Papkoff J, and Lambeth JD. Nox1 is overexpressed in human colon cancers and correlates with activating mutations in K-Ras. Int J Cancer 123: 100-107, 2008.

197. Laurila JP, Castellone MD, Curcio A, Laatikainen LE, Haaparanta-Solin M, Gronroos TJ, Marjamaki P, Martikainen S, Santoro M, and Laukkanen MO. Extracellular superoxide dismutase is a growth regulatory mediator of tissue injury recovery. Mol Ther 17: 448-454, 2009.

198. Laurila JP, Laatikainen LE, Castellone MD, and Laukkanen MO. SOD3 reduces inflammatory cell migration by regulating adhesion molecule and cytokine expression. PloS One 4: e5786, 2009.

199. Laurindo FR, Araujo TL, and Abrahao TB. Nox NADPH oxidases and the endoplasmic reticulum. Antioxid Redox Signal 20: 2755-2775, 2014.

200. Leal SS, Cardoso I, Valentine JS, and Gomes CM. Calcium ions promote superoxide dismutase 1 (SOD1) aggregation into non-fibrillar amyloid: a link to toxic effects of calcium overload in amyotrophic lateral sclerosis (ALS)? J Biol Chem 288: 25219-25228, 2013.

201. Lebovitz RM, Zhang H, Vogel H, Cartwright J, Jr., Dionne L, Lu N, Huang S, and Matzuk MM. Neurodegeneration, myocardial injury, and perinatal death in mitochondrial superoxide dismutase-deficient mice. Proc Natl Acad Sci U S A 93: 9782-9787, 1996.

202. Lemmon MA and Schlessinger J. Cell signaling by receptor tyrosine kinases. Cell 141: 1117-1134, 2010.

203. Leto TL, Adams AG, and de Mendez I. Assembly of the phagocyte NADPH oxidase: binding of Src homology 3 domains to proline-rich targets. Proc Natl Acad Sci U S A 91: 10650-10654, 1994.

204. Leung EL, Fan XX, Wong MP, Jiang ZH, Liu ZQ, Yao XJ, Lu LL, Zhou YL, Yau LF, Tin VP, and Liu L. Targeting tyrosine kinase inhibitor-resistant non-small cell lung cancer by inducing epidermal growth factor receptor degradation via methionine 790 oxidation. Antioxid Redox Signal 24: 263-279, 2016.

205. Li Z and Li L. Understanding hematopoietic stem-cell microenvironments. Trends Biochem Sci 31: 589-595, 2006.

206. Liang S, Kisseleva T, and Brenner DA. The role of NADPH oxidases (NOXs) in liver fibrosis and the activation of myofibroblasts. Front Physiol 7: 17, 2016.

207. Loo SY, Hirpara JL, Pandey V, Tan TZ, Yap CT, Lobie PE, Thiery JP, Goh BC, Pervaiz S, Clement MV, and 
Kumar AP. Manganese superoxide dismutase expression regulates the switch between an epithelial and a mesenchymal-like phenotype in breast carcinoma. Antioxid Redox Signal 25: 283-299, 2016.

208. Ludin A, Gur-Cohen S, Golan K, Kaufmann KB, Itkin T, Medaglia C, Lu XJ, Ledergor G, Kollet O, and Lapidot T. Reactive oxygen species regulate hematopoietic stem cell self-renewal, migration and development, as well as their bone marrow microenvironment. Antioxid Redox Signal 21: 1605-1619, 2014.

209. Lyle AN, Deshpande NN, Taniyama Y, Seidel-Rogol B, Pounkova L, Du P, Papaharalambus C, Lassegue B, and Griendling KK. Poldip2, a novel regulator of Nox4 and cytoskeletal integrity in vascular smooth muscle cells. Circ Res 105: 249-259, 2009.

210. Ma XJ, Dahiya S, Richardson E, Erlander M, and Sgroi DC. Gene expression profiling of the tumor microenvironment during breast cancer progression. Breast Cancer Res 11: R7, 2009.

211. Maehara Y, Miyano K, Yuzawa S, Akimoto R, Takeya R, and Sumimoto H. A conserved region between the TPR and activation domains of p67phox participates in activation of the phagocyte NADPH oxidase. J Biol Chem 285: 31435-31445, 2010.

212. Maekawa M, Ishizaki T, Boku S, Watanabe N, Fujita A, Iwamatsu A, Obinata T, Ohashi K, Mizuno K, and Narumiya S. Signaling from Rho to the actin cytoskeleton through protein kinases ROCK and LIM-kinase. Science 285: 895-898, 1999.

213. Magnani F, Nenci S, Millana Fananas E, Ceccon M, Romero E, Fraaije MW, and Mattevi A. Crystal structures and atomic model of NADPH oxidase. Proc Natl Acad Sci U S A 114: 6764-6769, 2017.

214. Maimon A, Mogilevsky M, Shilo A, Golan-Gerstl R, Obiedat A, Ben-Hur V, Lebenthal-Loinger I, Stein I, Reich R, Beenstock J, Zehorai E, Andersen CL, Thorsen K, Orntoft TF, Davis RJ, Davidson B, Mu D, and Karni R. Mnk2 alternative splicing modulates the p38-MAPK pathway and impacts Ras-induced transformation. Cell Rep 7: 501-513, 2014.

215. Malkin D, Li FP, Strong LC, Fraumeni JF, Jr., Nelson CE, Kim DH, Kassel J, Gryka MA, Bischoff FZ, Tainsky MA, and Friend $\mathrm{SH}$. Germ line p53 mutations in a familial syndrome of breast cancer, sarcomas, and other neoplasms. Science 250: 1233-1238, 1990.

216. Manickam N, Patel M, Griendling KK, Gorin Y, and Barnes JL. RhoA/Rho kinase mediates TGF-beta1induced kidney myofibroblast activation through Poldip2/ Nox4-derived reactive oxygen species. Am J Physiol Renal Physiol 307: F159-F171, 2014.

217. Mann T and Keilin D. Haemocuprein and hepatocuprein, copper-protein compounds of blood and liver in mammals. Proc R Soc Lond B Biol Sci 126: 303-315, 1938.

218. Mao X, Moerman-Herzog AM, Wang W, and Barger SW. Differential transcriptional control of the superoxide dismutase-2 kappaB element in neurons and astrocytes. $J$ Biol Chem 281: 35863-35872, 2006.

219. Maraldi T, Prata C, Caliceti C, Vieceli Dalla Sega F, Zambonin L, Fiorentini D, and Hakim G. VEGF-induced ROS generation from NAD $(\mathrm{P}) \mathrm{H}$ oxidases protects human leukemic cells from apoptosis. Int J Oncol 36: 1581-1589, 2010.

220. Maraldi T, Prata C, Vieceli Dalla Sega F, Caliceti C, Zambonin L, Fiorentini D, and Hakim G. NAD(P)H oxi- dase isoform Nox 2 plays a prosurvival role in human leukaemia cells. Free Radic Res 43: 1111-1121, 2009.

221. Marinissen MJ and Gutkind JS. G-protein-coupled receptors and signaling networks: emerging paradigms. Trends Pharmacol Sci 22: 368-376, 2001.

222. Marklund SL. Human copper-containing superoxide dismutase of high molecular weight. Proc Natl Acad Sci U S A 79: 7634-7638, 1982.

223. Marklund SL. Ceruloplasmin, extracellular-superoxide dismutase, and scavenging of superoxide anion radicals. $J$ Free Radic Biol Med 2: 255-260, 1986.

224. Marklund SL, Holme E, and Hellner L. Superoxide dismutase in extracellular fluids. Clin Chim Acta 126: 41-51, 1982.

225. Maryanovich M and Gross A. A ROS rheostat for cell fate regulation. Trends Cell Biol 23: 129-134, 2013.

226. Maryanovich M, Oberkovitz G, Niv H, Vorobiyov L, Zaltsman Y, Brenner O, Lapidot T, Jung S, and Gross A. The ATM-BID pathway regulates quiescence and survival of haematopoietic stem cells. Nat Cell Biol 14: 535-541, 2012.

227. Massart C, Hoste C, Virion A, Ruf J, Dumont JE, and Van Sande J. Cell biology of $\mathrm{H}_{2} \mathrm{O}_{2}$ generation in the thyroid: investigation of the control of dual oxidases (DUOX) activity in intact ex vivo thyroid tissue and cell lines. Mol Cell Endocrinol 343: 32-44, 2011.

228. Matsumoto S, Fujii S, Sato A, Ibuka S, Kagawa Y, Ishii $\mathrm{M}$, and Kikuchi A. A combination of Wnt and growth factor signaling induces Arl4c expression to form epithelial tubular structures. EMBO J 33: 702-718, 2014

229. Mayor R and Etienne-Manneville S. The front and rear of collective cell migration. Nat Rev Mol Cell Biol 17: 97109, 2016.

230. McCord JM and Fridovich I. Superoxide dismutase. An enzymic function for erythrocuprein (hemocuprein). $J$ Biol Chem 244: 6049-6055, 1969.

231. McKenzie RM, Johnson NA, Aruni W, Dou Y, Masinde G, and Fletcher HM. Differential response of Porphyromonas gingivalis to varying levels and duration of hydrogen peroxide-induced oxidative stress. Microbiology 158: 2465-2479, 2012.

232. Mead EL, Mosley A, Eaton S, Dobson L, Heales SJ, and Pocock JM. Microglial neurotransmitter receptors trigger superoxide production in microglia; consequences for microglial-neuronal interactions. J Neurochem 121: 287301, 2012.

233. Meijne EI, van der Winden-van Groenewegen RJ, Ploemacher RE, Vos O, David JA, and Huiskamp R. The effects of $\mathrm{x}$-irradiation on hematopoietic stem cell compartments in the mouse. Exp Hematol 19: 617-623, 1991.

234. Mendez-Ferrer S, Michurina TV, Ferraro F, Mazloom AR, Macarthur BD, Lira SA, Scadden DT, Ma'ayan A, Enikolopov GN, and Frenette PS. Mesenchymal and haematopoietic stem cells form a unique bone marrow niche. Nature 466: 829-834, 2010.

235. Meng A, Wang Y, Brown SA, Van Zant G, and Zhou D. Ionizing radiation and busulfan inhibit murine bone marrow cell hematopoietic function via apoptosis-dependent and -independent mechanisms. Exp Hematol 31: 13481356, 2003.

236. Meng D, Lv DD, and Fang J. Insulin-like growth factor-I induces reactive oxygen species production and cell migration through Nox4 and Rac1 in vascular smooth muscle cells. Cardiovasc Res 80: 299-308, 2008. 
237. Menshikov M, Plekhanova O, Cai H, Chalupsky K, Parfyonova Y, Bashtrikov P, Tkachuk V, and Berk BC. Urokinase plasminogen activator stimulates vascular smooth muscle cell proliferation via redox-dependent pathways. Arterioscler Thromb Vasc Biol 26: 801-807, 2006.

238. Miki Y, Swensen J, Shattuck-Eidens D, Futreal PA, Harshman K, Tavtigian S, Liu Q, Cochran C, Bennett LM, Ding W, Bell R, Rosenthal J, Hussey C, Tran T, McClure M, Frye C, Hattier T, Phelps R, Haugen-Strano A, Katcher H, Yakumo K, Gholami Z, Shaffer D, Stone S, Bayer S, Wray C, Bogden R, Dayananth P, Ward J, Tonin P, Narod S, Bristow PK, Norris FH, Helvering L, Morrison P, Rosteck P, Lai M, Barrett JC, Lewis C, Neuhausen S, Cannon-Albright L, Goldgar D, Wiseman R, Kramb A, and Skolnick MH. A strong candidate for the breast and ovarian cancer susceptibility gene BRCA1. Science 266: 66-71, 1994.

239. Miller EW, Dickinson BC, and Chang CJ. Aquaporin-3 mediates hydrogen peroxide uptake to regulate downstream intracellular signaling. Proc Natl Acad Sci U S A 107: 15681-15686, 2010.

240. Mitsushita J, Lambeth JD, and Kamata T. The superoxidegenerating oxidase Nox1 is functionally required for Ras oncogene transformation. Cancer Res 64: 3580-3585, 2004.

241. Miura Y, Gao Z, Miura M, Seo BM, Sonoyama W, Chen W, Gronthos S, Zhang L, and Shi S. Mesenchymal stem cellorganized bone marrow elements: an alternative hematopoietic progenitor resource. Stem Cells 24: 2428-2436, 2006.

242. Miyano K, Ueno N, Takeya R, and Sumimoto H. Direct involvement of the small GTPase Rac in activation of the superoxide-producing NADPH oxidase Nox1. J Biol Chem 281: 21857-21868, 2006.

243. Miyata Y, Matsuo T, Sagara Y, Ohba K, Ohyama K, and Sakai H. A mini-review of reactive oxygen species in urological cancer: correlation with NADPH oxidases, angiogenesis, and apoptosis. Int J Mol Sci 18: pii: E2214, 2017.

244. Mohr A, Buneker C, Gough RP, and Zwacka RM. MnSOD protects colorectal cancer cells from TRAILinduced apoptosis by inhibition of Smac/DIABLO release. Oncogene 27: 763-774, 2008.

245. Moloney JN, Stanicka J, and Cotter TG. Subcellular localization of the FLT3-ITD oncogene plays a significant role in the production of NOX- and p22(phox)-derived reactive oxygen species in acute myeloid leukemia. Leuk Res 52: 34-42, 2017.

246. Mondola P, Damiano S, Sasso A, and Santillo M. The Cu, Zn superoxide dismutase: not only a dismutase enzyme. Front Physiol 7: 594, 2016.

247. Mondola P, Santillo M, Seru R, Damiano S, Alvino C, Ruggiero G, Formisano P, Terrazzano G, Secondo A, and Annunziato $\mathrm{L}$. $\mathrm{Cu}, \mathrm{Zn}$ superoxide dismutase increases intracellular calcium levels via a phospholipase C-protein kinase C pathway in SK-N-BE neuroblastoma cells. Biochem Biophys Res Commun 324: 887-892, 2004.

248. Morrison SJ, Uchida N, and Weissman IL. The biology of hematopoietic stem cells. Annu Rev Cell Dev Biol 11: 3571, 1995.

249. Mueller S, Riedel HD, and Stremmel W. Determination of catalase activity at physiological hydrogen peroxide concentrations. Anal Biochem 245: 55-60, 1997.

250. Murphy MP. Modulating mitochondrial intracellular location as a redox signal. Sci Signal 5: pe39, 2012.

251. Nathke I. Cytoskeleton out of the cupboard: colon cancer and cytoskeletal changes induced by loss of APC. Nat Rev Cancer 6: 967-974, 2006.
252. Naughton R, Quiney C, Turner SD, and Cotter TG. BcrAbl-mediated redox regulation of the PI3K/AKT pathway. Leukemia 23: 1432-1440, 2009.

253. Nauseef WM. Detection of superoxide anion and hydrogen peroxide production by cellular NADPH oxidases. Biochim Biophys Acta 1840: 757-767, 2014.

254. Navarro-Yepes J, Burns M, Anandhan A, Khalimonchuk O, del Razo LM, Quintanilla-Vega B, Pappa A, Panayiotidis MI, and Franco R. Oxidative stress, redox signaling, and autophagy: cell death versus survival. Antioxid Redox Signal 21: 66-85, 2014.

255. Neben S, Marcus K, and Mauch P. Mobilization of hematopoietic stem and progenitor cell subpopulations from the marrow to the blood of mice following cyclophosphamide and/or granulocyte colony-stimulating factor. Blood 81: 1960-1967, 1993.

256. Nebl T, Oh SW, and Luna EJ. Membrane cytoskeleton: PIP(2) pulls the strings. Curr Biol 10: R351-R354, 2000.

257. Negrini S, Gorgoulis VG, and Halazonetis TD. Genomic instability-an evolving hallmark of cancer. Nat Rev Mol Cell Biol 11: 220-228, 2010.

258. Nelson KK, Ranganathan AC, Mansouri J, Rodriguez AM, Providence KM, Rutter JL, Pumiglia K, Bennett JA, and Melendez JA. Elevated SOD2 activity augments matrix metalloproteinase expression: evidence for the involvement of endogenous hydrogen peroxide in regulating metastasis. Clin Cancer Res 9: 424-432, 2003.

259. Nishiura T, Suzuki K, Kawaguchi T, Nakao H, Kawamura N, Taniguchi M, Kanayama Y, Yonezawa T, Iizuka S, and Taniguchi N. Elevated serum manganese superoxide dismutase in acute leukemias. Cancer Lett 62: 211-215, 1992.

260. Novo E, Marra F, Zamara E, Valfre di Bonzo L, Caligiuri A, Cannito S, Antonaci C, Colombatto S, Pinzani M, and Parola M. Dose dependent and divergent effects of superoxide anion on cell death, proliferation, and migration of activated human hepatic stellate cells. Gut 55: 90-97, 2006.

261. O'Hayre M, Degese MS, and Gutkind JS. Novel insights into $\mathrm{G}$ protein and $\mathrm{G}$ protein-coupled receptor signaling in cancer. Curr Opin Cell Biol 27: 126-135, 2014.

262. Oberley LW and Buettner GR. Role of superoxide dismutase in cancer: a review. Cancer Res 39: 1141-1149, 1979.

263. Ogrunc M, Di Micco R, Liontos M, Bombardelli L, Mione M, Fumagalli M, Gorgoulis VG, and d'Adda di Fagagna F. Oncogene-induced reactive oxygen species fuel hyperproliferation and DNA damage response activation. Cell Death Differ 21: 998-1012, 2014.

264. Oltra AM, Carbonell F, Tormos C, Iradi A, and Saez GT. Antioxidant enzyme activities and the production of MDA and 8 -oxo-dG in chronic lymphocytic leukemia. Free Radic Biol Med 30: 1286-1292, 2001.

265. Orkin SH and Zon LI. Hematopoiesis: an evolving paradigm for stem cell biology. Cell 132: 631-644, 2008.

266. Oshikawa J, Urao N, Kim HW, Kaplan N, Razvi M, McKinney R, Poole LB, Fukai T, and Ushio-Fukai M. Extracellular SOD-derived $\mathrm{H}_{2} \mathrm{O}_{2}$ promotes VEGF signaling in caveolae/lipid rafts and post-ischemic angiogenesis in mice. PloS One 5: e10189, 2010.

267. Ostman A, Frijhoff J, Sandin A, and Bohmer FD. Regulation of protein tyrosine phosphatases by reversible oxidation. J Biochem 150: 345-356, 2011.

268. Ozawa T, Araki N, Yunoue S, Tokuo H, Feng L, Patrakitkomjorn S, Hara T, Ichikawa Y, Matsumoto K, Fujii $\mathrm{K}$, and Saya $\mathrm{H}$. The neurofibromatosis type 1 gene product 
neurofibromin enhances cell motility by regulating actin filament dynamics via the Rho-ROCK-LIMK2-cofilin pathway. J Biol Chem 280: 39524-39533, 2005.

269. Paffenholz R, Bergstrom RA, Pasutto F, Wabnitz P, Munroe RJ, Jagla W, Heinzmann U, Marquardt A, Bareiss A, Laufs J, Russ A, Stumm G, Schimenti JC, and Bergstrom DE. Vestibular defects in head-tilt mice result from mutations in Nox3, encoding an NADPH oxidase. Genes Dev 18: 486-491, 2004.

270. Panday A, Sahoo MK, Osorio D, and Batra S. NADPH oxidases: an overview from structure to innate immunityassociated pathologies. Cell Mol Immunol 12: 5-23, 2015.

271. Pandey D and Fulton DJ. Molecular regulation of NADPH oxidase 5 via the MAPK pathway. Am J Physiol Heart Circ Physiol 300: H1336-H1344, 2011.

272. Pandey D, Gratton JP, Rafikov R, Black SM, and Fulton DJ. Calcium/calmodulin-dependent kinase II mediates the phosphorylation and activation of NADPH oxidase 5. Mol Pharmacol 80: 407-415, 2011.

273. Papa L, Hahn M, Marsh EL, Evans BS, and Germain D. SOD2 to SOD1 switch in breast cancer. J Biol Chem 289: 5412-5416, 2014.

274. Papa L, Manfredi G, and Germain D. SOD1, an unexpected novel target for cancer therapy. Genes Cancer 5: 15-21, 2014.

275. Parascandolo A, Rappa F, Cappello F, Kim J, Cantu DA, Chen H, Mazzoccoli G, Hematti P, Castellone MD, Salvatore $\mathrm{M}$, and Laukkanen MO. Extracellular superoxide dismutase expression in papillary thyroid cancer mesenchymal stem/stromal cells modulates cancer cell growth and migration. Sci Rep 7: 41416, 2017.

276. Park EY and Rho HM. The transcriptional activation of the human copper/zinc superoxide dismutase gene by 2,3,7,8-tetrachlorodibenzo-p-dioxin through two different regulator sites, the antioxidant responsive element and xenobiotic responsive element. Mol Cell Biochem 240: 47-55, 2002.

277. Park JW and Babior BM. Activation of the leukocyte NADPH oxidase subunit p47phox by protein kinase C. A phosphorylation-dependent change in the conformation of the C-terminal end of p47phox. Biochemistry 36: 74747480, 1997.

278. Park MT, Kim MJ, Suh Y, Kim RK, Kim H, Lim EJ, Yoo KC, Lee GH, Kim YH, Hwang SG, Yi JM, and Lee SJ. Novel signaling axis for ROS generation during K-Rasinduced cellular transformation. Cell Death Differ 21: 1185-1197, 2014.

279. Park SY and Bae YS. Inactivation of the FoxO3a transcription factor is associated with the production of reactive oxygen species during protein kinase CK2 downregulationmediated senescence in human colon cancer and breast cancer cells. Biochem Biophys Res Commun 478: 18-24, 2016.

280. Parkos CA, Allen RA, Cochrane CG, and Jesaitis AJ. Purified cytochrome b from human granulocyte plasma membrane is comprised of two polypeptides with relative molecular weights of 91,000 and 22,000. J Clin Invest 80: 732-742, 1987.

281. Pawlak G and Helfman DM. MEK mediates v-Srcinduced disruption of the actin cytoskeleton via inactivation of the Rho-ROCK-LIM kinase pathway. J Biol Chem 277: 26927-26933, 2002.

282. Pazhanisamy SK, Li H, Wang Y, Batinic-Haberle I, and Zhou D. NADPH oxidase inhibition attenuates total body irradiation-induced haematopoietic genomic instability. Mutagenesis 26: 431-435, 2011.

283. Pellegrini M, Cheng JC, Voutila J, Judelson D, Taylor J, Nelson SF, and Sakamoto KM. Expression profile of CREB knockdown in myeloid leukemia cells. $B M C$ Cancer 8: 264, 2008.

284. Petkau A. Role of superoxide dismutase in modification of radiation injury. Br J Cancer Suppl 8: 87-95, 1987.

285. Piccoli C, D’Aprile A, Ripoli M, Scrima R, Boffoli D, Tabilio A, and Capitanio N. The hypoxia-inducible factor is stabilized in circulating hematopoietic stem cells under normoxic conditions. FEBS Lett 581: 3111-3119, 2007.

286. Piccoli C, D’Aprile A, Ripoli M, Scrima R, Lecce L, Boffoli D, Tabilio A, and Capitanio N. Bone-marrow derived hematopoietic stem/progenitor cells express multiple isoforms of NADPH oxidase and produce constitutively reactive oxygen species. Biochem Biophys Res Commun 353: 965-972, 2007.

287. Pick E, Gorzalczany Y, and Engel S. Role of the rac1 p21GDP-dissociation inhibitor for rho heterodimer in the activation of the superoxide-forming NADPH oxidase of macrophages. Eur J Biochem 217: 441-455, 1993.

288. Piecuch A, Brzozowa-Zasada M, Dziewit B, Segiet O, Kurek J, Kowalczyk-Ziomek G, Wojnicz R, and Helewski $\mathrm{K}$. Immunohistochemical assessment of mitochondrial superoxide dismutase (MnSOD) in colorectal premalignant and malignant lesions. Prz Gastroenterol 11: 239246, 2016.

289. Plymate SR, Haugk KH, Sprenger CC, Nelson PS, Tennant MK, Zhang Y, Oberley LW, Zhong W, Drivdahl R, and Oberley TD. Increased manganese superoxide dismutase (SOD-2) is part of the mechanism for prostate tumor suppression by Mac25/insulin-like growth factor bindingprotein-related protein-1. Oncogene 22: 1024-1034, 2003.

290. Porter H and Folch J. Cerebrocuprein I. A coppercontaining protein isolated from brain. J Neurochem 1: 260-271, 1957.

291. Porto ML, Rodrigues BP, Menezes TN, Ceschim SL, Casarini DE, Gava AL, Pereira TM, Vasquez EC, Campagnaro BP, and Meyrelles SS. Reactive oxygen species contribute to dysfunction of bone marrow hematopoietic stem cells in aged C57BL/6 J mice. J Biomed Sci 22: 97, 2015.

292. Premont RT and Gainetdinov RR. Physiological roles of $\mathrm{G}$ protein-coupled receptor kinases and arrestins. Аnпи Rev Physiol 69: 511-534, 2007.

293. Puca R, Nardinocchi L, Starace G, Rechavi G, Sacchi A, Givol D, and D'Orazi G. Nox1 is involved in p53 deacetylation and suppression of its transcriptional activity and apoptosis. Free Radic Biol Med 48: 1338-1346, 2010.

294. Rameh LE, Rhee SG, Spokes K, Kazlauskas A, Cantley LC, and Cantley LG. Phosphoinositide 3-kinase regulates phospholipase Cgamma-mediated calcium signaling. $J$ Biol Chem 273: 23750-23757, 1998.

295. Ran S. The role of TLR4 in chemotherapy-driven metastasis. Cancer Res 75: 2405-2410, 2015.

296. Rassool FV, Gaymes TJ, Omidvar N, Brady N, Beurlet S, Pla M, Reboul M, Lea N, Chomienne C, Thomas NS, Mufti GJ, and Padua RA. Reactive oxygen species, DNA damage, and error-prone repair: a model for genomic instability with progression in myeloid leukemia? Cancer Res 67: 8762-8771, 2007.

297. Reddi AR and Culotta VC. SOD1 integrates signals from oxygen and glucose to repress respiration. Cell 152: 224$235,2013$. 
298. Reddy MM, Fernandes MS, Deshpande A, Weisberg E, Inguilizian HV, Abdel-Wahab O, Kung AL, Levine RL, Griffin JD, and Sattler M. The JAK2V617F oncogene requires expression of inducible phosphofructokinase/ fructose-bisphosphatase 3 for cell growth and increased metabolic activity. Leukemia 26: 481-489, 2012.

299. Redza-Dutordoir M and Averill-Bates DA. Activation of apoptosis signalling pathways by reactive oxygen species. Biochim Biophys Acta 1863: 2977-2992, 2016.

300. Riento K and Ridley AJ. Rocks: multifunctional kinases in cell behaviour. Nat Rev Mol Cell Biol 4: 446-456, 2003.

301. Rodrigues-Moreira S, Moreno SG, Ghinatti G, Lewandowski D, Hoffschir F, Ferri F, Gallouet AS, Gay D, Motohashi H, Yamamoto M, Joiner MC, Gault N, and Romeo PH. Low-dose irradiation promotes persistent oxidative stress and decreases self-renewal in hematopoietic stem cells. Cell Rep 20: 3199-3211, 2017.

302. Rosen DR, Siddique T, Patterson D, Figlewicz DA, Sapp P, Hentati A, Donaldson D, Goto J, O'Regan JP, Deng HX, Rahmani Z, Krizus A, McKenna-Yasek D, Cayabyab A, Gaston SM, Berger R, Tanzi RE, Halperin JJ, Herzfeldt B, Van den Bergh R, Hung WY, Bird T, Deng G, Mulder DW, Smyth C, Laing NG, Soriano E, Pericak-Vance MA, Haines J, Rouleau GA, Gusella JS, Horvitz HR, and Brown Jr RH. Mutations in $\mathrm{Cu} / \mathrm{Zn}$ superoxide dismutase gene are associated with familial amyotrophic lateral sclerosis. Nature 362: 59-62, 1993.

303. Rossi DJ, Seita J, Czechowicz A, Bhattacharya D, Bryder $\mathrm{D}$, and Weissman IL. Hematopoietic stem cell quiescence attenuates DNA damage response and permits DNA damage accumulation during aging. Cell Cycle 6: 23712376, 2007.

304. Rotow $\mathrm{J}$ and Bivona TG. Understanding and targeting resistance mechanisms in NSCLC. Nat Rev Cancer 17: 637-658, 2017.

305. Sabates-Bellver J, Van der Flier LG, de Palo M, Cattaneo E, Maake C, Rehrauer H, Laczko E, Kurowski MA, Bujnicki JM, Menigatti M, Luz J, Ranalli TV, Gomes V, Pastorelli A, Faggiani R, Anti M, Jiricny J, Clevers H, and Marra G. Transcriptome profile of human colorectal adenomas. Mol Cancer Res 5: 1263-1275, 2007.

306. Sacchetti B, Funari A, Michienzi S, Di Cesare S, Piersanti S, Saggio I, Tagliafico E, Ferrari S, Robey PG, Riminucci $\mathrm{M}$, and Bianco P. Self-renewing osteoprogenitors in bone marrow sinusoids can organize a hematopoietic microenvironment. Cell 131: 324-336, 2007.

307. Sack MN, Fyhrquist FY, Saijonmaa OJ, Fuster V, and Kovacic JC. Basic biology of oxidative stress and the cardiovascular system: part 1 of a 3-part series. J Am Coll Cardiol 70: 196-211, 2017.

308. Sadok A, Bourgarel-Rey V, Gattacceca F, Penel C, Lehmann $M$, and Kovacic H. Nox1-dependent superoxide production controls colon adenocarcinoma cell migration. Biochim Biophys Acta 1783: 23-33, 2008.

309. Salazar-Roa M and Malumbres M. Fueling the cell division cycle. Trends Cell Biol 27: 69-81, 2017.

310. Sallmyr A, Fan J, Datta K, Kim KT, Grosu D, Shapiro P, Small D, and Rassool F. Internal tandem duplication of FLT3 (FLT3/ITD) induces increased ROS production, DNA damage, and misrepair: implications for poor prognosis in AML. Blood 111: 3173-3182, 2008.

311. Sallmyr A, Fan J, and Rassool FV. Genomic instability in myeloid malignancies: increased reactive oxygen species
(ROS), DNA double strand breaks (DSBs) and error-prone repair. Cancer Lett 270: 1-9, 2008.

312. Sampson N, Koziel R, Zenzmaier C, Bubendorf L, Plas E, Jansen-Durr P, and Berger P. ROS signaling by NOX4 drives fibroblast-to-myofibroblast differentiation in the diseased prostatic stroma. Mol Endocrinol 25: 503-515, 2011.

313. Sanders LM, Henderson CE, Hong MY, Barhoumi R, Burghardt RC, Carroll RJ, Turner ND, Chapkin RS, and Lupton JR. Pro-oxidant environment of the colon compared to the small intestine may contribute to greater cancer susceptibility. Cancer Lett 208: 155-161, 2004.

314. Sandstrom J, Carlsson L, Marklund SL, and Edlund T. The heparin-binding domain of extracellular superoxide dismutase $\mathrm{C}$ and formation of variants with reduced heparin affinity. J Biol Chem 267: 18205-18209, 1992.

315. Sarkisian CJ, Keister BA, Stairs DB, Boxer RB, Moody SE, and Chodosh LA. Dose-dependent oncogene-induced senescence in vivo and its evasion during mammary tumorigenesis. Nat Cell Biol 9: 493-505, 2007.

316. Scharenberg AM and Kinet JP. PtdIns-3,4,5-P3: a regulatory nexus between tyrosine kinases and sustained calcium signals. Cell 94: 5-8, 1998.

317. Scheuba C, Kaserer K, Kaczirek K, Asari R, and Niederle B. Desmoplastic stromal reaction in medullary thyroid cancer-an intraoperative "marker" for lymph node metastases. World J Surg 30: 853-859, 2006.

318. Segal AW, Dorling J, and Coade S. Kinetics of fusion of the cytoplasmic granules with phagocytic vacuoles in human polymorphonuclear leukocytes. Biochemical and morphological studies. J Cell Biol 85: 42-59, 1980.

319. Senturker S, Karahalil B, Inal M, Yilmaz H, Muslumanoglu H, Gedikoglu G, and Dizdaroglu M. Oxidative DNA base damage and antioxidant enzyme levels in childhood acute lymphoblastic leukemia. FEBS Lett 416: 286-290, 1997.

320. Seo SJ, Kim HT, Cho G, Rho HM, and Jung G. Sp1 and $\mathrm{C} / \mathrm{EBP}$-related factor regulate the transcription of human $\mathrm{Cu} / \mathrm{Zn}$ SOD gene. Gene 178: 177-185, 1996.

321. Serrano M, Gomez-Lahoz E, DePinho RA, Beach D, and Bar-Sagi D. Inhibition of ras-induced proliferation and cellular transformation by p16INK4. Science 267: 249252, 1995.

322. Serrano M, Lin AW, McCurrach ME, Beach D, and Lowe $\mathrm{SW}$. Oncogenic ras provokes premature cell senescence associated with accumulation of p53 and p16INK4a. Cell 88: 593-602, 1997.

323. Servitja JM, Marinissen MJ, Sodhi A, Bustelo XR, and Gutkind JS. Rac1 function is required for Src-induced transformation. Evidence of a role for Tiam1 and Vav2 in Rac activation by Src. J Biol Chem 278: 34339-34346, 2003.

324. Shao L, Luo Y, and Zhou D. Hematopoietic stem cell injury induced by ionizing radiation. Antioxid Redox Signal 20: 1447-1462, 2014.

325. Sharma LK, Lu J, and Bai Y. Mitochondrial respiratory complex I: structure, function and implication in human diseases. Curr Med Chem 16: 1266-1277, 2009.

326. Shatwell KP and Segal AW. NADPH oxidase. Int J Biochem Cell Biol 28: 1191-1195, 1996.

327. Shibata N, Hirano A, Yamamoto T, Kato Y, and Kobayashi M. Superoxide dismutase-1 mutation-related neurotoxicity in familial amyotrophic lateral sclerosis. Amyotroph Lateral Scler Other Motor Neuron Disord 1: 143-161, 2000. 
328. Shigemura T, Shiohara M, Kato M, Furuta S, Kaneda K, Morishita K, Hasegawa H, Fujii M, Gorlach A, Koike K, and Kamata T. Superoxide-generating Nox5alpha is functionally required for the human T-cell leukemia virus type 1-induced cell transformation phenotype. J Virol 89: 9080-9089, 2015.

329. Shimada K, Fujii T, Anai S, Fujimoto K, and Konishi N. ROS generation via NOX4 and its utility in the cytological diagnosis of urothelial carcinoma of the urinary bladder. BMC Urol 11: 22, 2011.

330. Shinohara M, Adachi Y, Mitsushita J, Kuwabara M, Nagasawa A, Harada S, Furuta S, Zhang Y, Seheli K, Miyazaki $\mathrm{H}$, and Kamata $\mathrm{T}$. Reactive oxygen generated by NADPH oxidase 1 (Nox1) contributes to cell invasion by regulating matrix metalloprotease- 9 production and cell migration. J Biol Chem 285: 4481-4488, 2010.

331. Si J, Behar J, Wands J, Beer DG, Lambeth D, Chin YE, and Cao W. STAT5 mediates PAF-induced NADPH oxidase NOX5-S expression in Barrett's esophageal adenocarcinoma cells. Am J Physiol Gastrointest Liver Physiol 294: G174-G183, 2008.

332. Sjoquist PO and Marklund SL. Endothelium bound extracellular superoxide dismutase type $\mathrm{C}$ reduces damage in reperfused ischaemic rat hearts. Cardiovasc Res 26: 347-350, 1992.

333. Slauch JM. How does the oxidative burst of macrophages kill bacteria? Still an open question. Mol Microbiol 80: 580-583, 2011.

334. Smolinska MJ, Horwood NJ, Page TH, Smallie T, and Foxwell BM. Chemical inhibition of Src family kinases affects major LPS-activated pathways in primary human macrophages. Mol Immunol 45: 990-1000, 2008.

335. Song IS, Jeong YJ, Nyamaa B, Jeong SH, Kim HK, Kim N, Ko KS, Rhee BD, and Han J. KSP inhibitor SB743921 induces death of multiple myeloma cells via inhibition of the NF-kappaB signaling pathway. BMB Rep 48: 571-576, 2015.

336. Song Y, Driessens N, Costa M, De Deken X, Detours V, Corvilain B, Maenhaut C, Miot F, Van Sande J, Many $\mathrm{MC}$, and Dumont JE. Roles of hydrogen peroxide in thyroid physiology and disease. J Clin Endocrinol Metab 92: 3764-3773, 2007.

337. Sorescu GP, Song H, Tressel SL, Hwang J, Dikalov S, Smith DA, Boyd NL, Platt MO, Lassegue B, Griendling $\mathrm{KK}$, and Jo H. Bone morphogenic protein 4 produced in endothelial cells by oscillatory shear stress induces monocyte adhesion by stimulating reactive oxygen species production from a nox1-based NADPH oxidase. Circ Res 95: 773-779, 2004.

338. Stanicka J, Russell EG, Woolley JF, and Cotter TG. NADPH oxidase-generated hydrogen peroxide induces DNA damage in mutant FLT3-expressing leukemia cells. J Biol Chem 290: 9348-9361, 2015.

339. Stone JR. An assessment of proposed mechanisms for sensing hydrogen peroxide in mammalian systems. Arch Biochem Biophys 422: 119-124, 2004.

340. Stone JR and Yang S. Hydrogen peroxide: a signaling messenger. Antioxid Redox Signal 8: 243-270, 2006.

341. Strilic B and Offermanns S. Intravascular survival and extravasation of tumor cells. Cancer Cell 32: 282-293, 2017.

342. Suh YA, Arnold RS, Lassegue B, Shi J, Xu X, Sorescu D, Chung AB, Griendling KK, and Lambeth JD. Cell transformation by the superoxide-generating oxidase Mox 1 . Nature 401: 79-82, 1999.
343. Sumimoto H, Kage Y, Nunoi H, Sasaki H, Nose T, Fukumaki Y, Ohno M, Minakami S, and Takeshige K. Role of Src homology 3 domains in assembly and activation of the phagocyte NADPH oxidase. Proc Natl Acad Sci U S A 91: 5345-5349, 1994.

344. Taddei ML, Giannoni E, Fiaschi T, and Chiarugi P. Anoikis: an emerging hallmark in health and diseases. $J$ Pathol 226: 380-393, 2012.

345. Takac I, Schroder K, Zhang L, Lardy B, Anilkumar N, Lambeth JD, Shah AM, Morel F, and Brandes RP. The E-loop is involved in hydrogen peroxide formation by the NADPH oxidase Nox4. J Biol Chem 286: 13304-13313, 2011.

346. Teoh ML, Fitzgerald MP, Oberley LW, and Domann FE. Overexpression of extracellular superoxide dismutase attenuates heparanase expression and inhibits breast carcinoma cell growth and invasion. Cancer Res 69: 63556363, 2009.

347. Teoh ML, Sun W, Smith BJ, Oberley LW, and Cullen JJ. Modulation of reactive oxygen species in pancreatic cancer. Clin Cancer Res 13: 7441-7450, 2007.

348. Theccanat T, Philip JL, Razzaque AM, Ludmer N, Li J, $\mathrm{Xu} \mathrm{X}$, and Akhter SA. Regulation of cellular oxidative stress and apoptosis by $\mathrm{G}$ protein-coupled receptor kinase2; the role of NADPH oxidase 4. Cell Signal 28: 190-203, 2016.

349. Thomsen AR, Plouffe B, Cahill TJ, 3rd, Shukla AK, Tarrasch JT, Dosey AM, Kahsai AW, Strachan RT, Pani B, Mahoney JP, Huang L, Breton B, Heydenreich FM, Sunahara RK, Skiniotis G, Bouvier M, and Lefkowitz RJ. GPCR-G protein-beta-arrestin super-complex mediates sustained G protein signaling. Cell 166: 907-919, 2016.

350. Tobar N, Guerrero J, Smith PC, and Martinez J. NOX4dependent ROS production by stromal mammary cells modulates epithelial MCF-7 cell migration. Br J Cancer 103: 1040-1047, 2010.

351. Tobar N, Toyos M, Urra C, Mendez N, Arancibia R, Smith PC, and Martinez J. c-Jun N terminal kinase modulates NOX-4 derived ROS production and myofibroblasts differentiation in human breast stromal cells. BMC Cancer 14: 640, 2014.

352. Tojo T, Ushio-Fukai M, Yamaoka-Tojo M, Ikeda S, Patrushev N, and Alexander RW. Role of gp91phox (Nox2)containing $\mathrm{NAD}(\mathrm{P}) \mathrm{H}$ oxidase in angiogenesis in response to hindlimb ischemia. Circulation 111: 2347-2355, 2005.

353. Tomoda T, Nomura I, Kurashige T, Kubonishi I, Miyoshi I, Sukenaga $\mathrm{Y}$, and Taniguchi $\mathrm{T}$. Changes in $\mathrm{Cu}, \mathrm{Zn}$ superoxide dismutase gene during induced erythroid and myeloid differentiation. Acta Haematol 86: 183-188, 1991.

354. Tonks NK. Redox redux: revisiting PTPs and the control of cell signaling. Cell 121: 667-670, 2005.

355. Tosi F, Magni E, Amatu A, Mauri G, Bencardino K, Truini M, Veronese S, De Carlis L, Ferrari G, Nichelatti M, Sartore-Bianchi A, and Siena S. Effect of KRAS and BRAF mutations on survival of metastatic colorectal cancer after liver resection: a systematic review and metaanalysis. Clin Colorectal Cancer 16: e153-e163, 2017.

356. Tothova Z, Kollipara R, Huntly BJ, Lee BH, Castrillon DH, Cullen DE, McDowell EP, Lazo-Kallanian S, Williams IR, Sears C, Armstrong SA, Passegue E, DePinho RA, and Gilliland DG. FoxOs are critical mediators of hematopoietic stem cell resistance to physiologic oxidative stress. Cell 128: 325-339, 2007.

357. Uchida N and Weissman IL. Searching for hematopoietic stem cells: evidence that Thy-1.1lo Lin- $\mathrm{Sca}-1^{+}$cells are 
the only stem cells in C57BL/Ka-Thy-1.1 bone marrow. $J$ Exp Med 175: 175-184, 1992.

358. Ueno N, Takeya R, Miyano K, Kikuchi H, and Sumimoto H. The NADPH oxidase Nox3 constitutively produces superoxide in a 22 phox-dependent manner: its regulation by oxidase organizers and activators. J Biol Chem 280: 23328-23339, 2005.

359. Ueyama T, Nakakita J, Nakamura T, Kobayashi T, Kobayashi T, Son J, Sakuma M, Sakaguchi H, Leto TL, and Saito N. Cooperation of p40(phox) with p47(phox) for Nox2-based NADPH oxidase activation during Fcgamma receptor (FcgammaR)-mediated phagocytosis: mechanism for acquisition of p40(phox) phosphatidylinositol 3phosphate (PI(3)P) binding. J Biol Chem 286: 4069340705, 2011.

360. Ueyama T, Tatsuno T, Kawasaki T, Tsujibe S, Shirai Y, Sumimoto H, Leto TL, and Saito N. A regulated adaptor function of p40phox: distinct p67phox membrane targeting by p40phox and by p47phox. Mol Biol Cell 18: 441454, 2007.

361. Ungefroren $H$, Witte $D$, and Lehnert $H$. The role of small GTPases of the Rho/Rac family in TGF-beta-induced EMT and cell motility in cancer. Dev Dyn 247: 451-461, 2018.

362. Valk PJ, Verhaak RG, Beijen MA, Erpelinck CA, Barjesteh van Waalwijk van Doorn-Khosrovani S, Boer JM, Beverloo HB, Moorhouse MJ, van der Spek PJ, Lowenberg B, and Delwel R. Prognostically useful geneexpression profiles in acute myeloid leukemia. $N$ Engl $J$ Med 350: 1617-1628, 2004.

363. Van Der Heide LP, Hoekman MF, and Smidt MP. The ins and outs of FoxO shuttling: mechanisms of FoxO translocation and transcriptional regulation. Biochem $J$ 380: 297-309, 2004.

364. Vecchio G, Parascandolo A, Allocca C, Ugolini C, Basolo F, Moracci M, Strazzulli A, Cobucci-Ponzano B, Laukkanen MO, Castellone MD, and Tsuchida N. Human a-Lfucosidase-1 attenuates the invasive properties of thyroid cancer. Oncotarget 8: 27075-27092, 2017.

365. Venza M, Visalli M, Beninati C, De Gaetano GV, Teti D, and Venza I. Cellular mechanisms of oxidative stress and action in melanoma. Oxid Med Cell Longev 2015: 481782, 2015.

366. Wagner BA, Witmer JR, van 't Erve TJ, and Buettner GR. An assay for the rate of removal of extracellular hydrogen peroxide by cells. Redox Biol 1: 210-217, 2013.

367. Wagner EF and Nebreda AR. Signal integration by JNK and p38 MAPK pathways in cancer development. Nat Rev Cancer 9: 537-549, 2009.

368. Wang CA, Harrell JC, Iwanaga R, Jedlicka P, and Ford HL. Vascular endothelial growth factor $C$ promotes breast cancer progression via a novel antioxidant mechanism that involves regulation of superoxide dismutase 3. Breast Cancer Res 16: 462, 2014.

369. Wang Y, Chen F, Le B, Stepp DW, and Fulton DJ. Impact of Nox5 polymorphisms on basal and stimulus-dependent ROS generation. PLoS One 9: e100102, 2014.

370. Waning DL, Mohammad KS, Reiken S, Xie W, Andersson DC, John S, Chiechi A, Wright LE, Umanskaya A, Niewolna M, Trivedi T, Charkhzarrin S, Khatiwada P, Wronska A, Haynes A, Benassi MS, Witzmann FA, Zhen G, Wang X, Cao X, Roodman GD, Marks AR, and Guise TA. Excess TGF-beta mediates muscle weakness associated with bone metastases in mice. Nat Med 21: 1262-1271, 2015.
371. Ward RA, Nakamura M, and McLeish KR. Priming of the neutrophil respiratory burst involves $\mathrm{p} 38$ mitogen-activated protein kinase-dependent exocytosis of flavocytochrome b558-containing granules. J Biol Chem 275: 36713-36719, 2000.

372. Wartenberg M, Diedershagen H, Hescheler J, and Sauer $H$. Growth stimulation versus induction of cell quiescence by hydrogen peroxide in prostate tumor spheroids is encoded by the duration of the $\mathrm{Ca}(2+)$ response. J Biol Chem 274: 27759-27767, 1999.

373. Weiss CN and Ito K. DNA damage: a sensible mediator of the differentiation decision in hematopoietic stem cells and in leukemia. Int J Mol Sci 16: 6183-6201, 2015.

374. Weyemi U, Caillou B, Talbot M, Ameziane-El-Hassani R, Lacroix L, Lagent-Chevallier O, Al Ghuzlan A, Roos D, Bidart JM, Virion A, Schlumberger M, and Dupuy C. Intracellular expression of reactive oxygen speciesgenerating NADPH oxidase NOX4 in normal and cancer thyroid tissues. Endocr Relat Cancer 17: 27-37, 2010.

375. Weyemi U, Lagente-Chevallier O, Boufraqech M, Prenois F, Courtin F, Caillou B, Talbot M, Dardalhon M, Al Ghuzlan A, Bidart JM, Schlumberger M, and Dupuy C. ROS-generating NADPH oxidase NOX4 is a critical mediator in oncogenic H-Ras-induced DNA damage and subsequent senescence. Oncogene 31: 1117-1129, 2012.

376. Weyemi U, Redon CE, Aziz T, Choudhuri R, Maeda D, Parekh PR, Bonner MY, Arbiser JL, and Bonner WM. Inactivation of NADPH oxidases NOX4 and NOX5 protects human primary fibroblasts from ionizing radiationinduced DNA damage. Radiat Res 183: 262-270, 2015.

377. Wheeler MD, Smutney OM, and Samulski RJ. Secretion of extracellular superoxide dismutase from muscle transduced with recombinant adenovirus inhibits the growth of B16 melanomas in mice. Mol Cancer Res 1: 871-881, 2003.

378. Wieczorek E, Jablonowski Z, Tomasik B, Gromadzinska J, Jablonska E, Konecki T, Fendler W, Sosnowski M, Wasowicz W, and Reszka E. Different gene expression and activity pattern of antioxidant enzymes in bladder cancer. Anticancer Res 37: 841-848, 2017.

379. Willems J, Zwijsen A, Slegers H, Nicolai S, Bettadapura J, Raymackers J, and Scarcez T. Purification and sequence of rat extracellular superoxide dismutase B secreted by C6 glioma. J Biol Chem 268: 24614-24621, 1993.

380. Winter WE and Signorino MR. Review: molecular thyroidology. Ann Clin Lab Sci 31: 221-244, 2001.

381. Wirth C, Brandt U, Hunte C, and Zickermann V. Structure and function of mitochondrial complex I. Biochim Biophys Acta 1857: 902-914, 2016.

382. Wynn TA and Ramalingam TR. Mechanisms of fibrosis: therapeutic translation for fibrotic disease. Nat Med 18: 1028-1040, 2012.

383. Xing M. Molecular pathogenesis and mechanisms of thyroid cancer. Nat Rev Cancer 13: 184-199, 2013.

384. Xu Y, Miriyala S, Fang F, Bakthavatchalu V, Noel T, Schnell DM, Wang C, St Clair WH, and St Clair DK. Manganese superoxide dismutase deficiency triggers mitochondrial uncoupling and the Warburg effect. Oncogene 36: 4087, 2017.

385. Xu Y, Porntadavity S, and St Clair DK. Transcriptional regulation of the human manganese superoxide dismutase gene: the role of specificity protein 1 (Sp1) and activating protein-2 (AP-2). Biochem J 362: 401-412, 2002.

386. Yahata $\mathrm{T}$, Takanashi $\mathrm{T}$, Muguruma $\mathrm{Y}$, Ibrahim AA, Matsuzawa H, Uno T, Sheng Y, Onizuka M, Ito M, Kato 
S, and Ando K. Accumulation of oxidative DNA damage restricts the self-renewal capacity of human hematopoietic stem cells. Blood 118: 2941-2950, 2011.

387. Yamaguchi S, Sakurada S, and Nagumo M. Role of intracellular SOD in protecting human leukemic and cancer cells against superoxide and radiation. Free Radic Biol Med 17: 389-395, 1994.

388. Yamamoto A, Takeya R, Matsumoto M, Nakayama KI, and Sumimoto H. Phosphorylation of NOXO1 at threonine 341 regulates its interaction with Noxal and the superoxide-producing activity of Nox1. FEBS $J$ 280: 5145-5159, 2013.

389. Yamaura M, Mitsushita J, Furuta S, Kiniwa Y, Ashida A, Goto Y, Shang WH, Kubodera M, Kato M, Takata M, Saida T, and Kamata T. NADPH oxidase 4 contributes to transformation phenotype of melanoma cells by regulating G2-M cell cycle progression. Cancer Res 69: 2647-2654, 2009.

390. Yi J, Kloeker S, Jensen CC, Bockholt S, Honda H, Hirai $\mathrm{H}$, and Beckerle MC. Members of the Zyxin family of LIM proteins interact with members of the p130Cas family of signal transducers. J Biol Chem 277: 95809589, 2002.

391. Yla-Herttuala S, Luoma J, Kallionpaa H, Laukkanen M, Lehtolainen P, and Viita H. Pathogenesis of atherosclerosis. Maturitas 29(Suppl): S47-S49, 1996.

392. Young B, Purcell C, Kuang YQ, Charette N, and Dupre DJ. Superoxide dismutase 1 regulation of CXCR4mediated signaling in prostate cancer cells is dependent on cellular oxidative state. Cell Physiol Biochem 37: 20712084, 2015.

393. Zelko IN and Folz RJ. Sp1 and Sp3 transcription factors mediate trichostatin A-induced and basal expression of extracellular superoxide dismutase. Free Radic Biol Med 37: 1256-1271, 2004.

394. Zelko IN, Mueller MR, and Folz RJ. CpG methylation attenuates Sp1 and Sp3 binding to the human extracellular superoxide dismutase promoter and regulates its cell-specific expression. Free Radic Biol Med 48: 895-904, 2010.

395. Zhang HJ, Zhao W, Venkataraman S, Robbins ME, Buettner GR, Kregel KC, and Oberley LW. Activation of matrix metalloproteinase- 2 by overexpression of manganese superoxide dismutase in human breast cancer MCF-7 cells involves reactive oxygen species. J Biol Chem 277: 20919-20926, 2002.

396. Zhang X, Ng WL, Wang P, Tian L, Werner E, Wang H, Doetsch P, and Wang Y. MicroRNA-21 modulates the levels of reactive oxygen species by targeting SOD3 and TNFalpha. Cancer Res 72: 4707-4713, 2012.

397. Zhong W, Oberley LW, Oberley TD, and St Clair DK. Suppression of the malignant phenotype of human glioma cells by overexpression of manganese superoxide dismutase. Oncogene 14: 481-490, 1997.

398. Zhou BB and Elledge SJ. The DNA damage response: putting checkpoints in perspective. Nature 408: 433-439, 2000 .

399. Zimmermann R, Flohe L, Weser U, and Hartmann HJ. Inhibition of lipid peroxidation in isolated inner membrane of rat liver mitochondria by superoxide dismutase. FEBS Lett 29: 117-120, 1973.

400. Zuo XL, Chen JM, Zhou X, Li XZ, and Mei GY. Levels of selenium, zinc, copper, and antioxidant enzyme activity in patients with leukemia. Biol Trace Elem Res 114: 41-53, 2006.
Address correspondence to: Dr. Mikko O. Laukkanen

Institute of Experimental Endocrinolgy and Oncology (CNR)

Via Sergio Pansini 5 80014 Naples

Italy

E-mail: m.laukkan@yahoo.com

Date of first submission to ARS Central, July 5, 2017; date of final revised submission, February 21, 2018; date of acceptance, February 22, 2018.

\begin{tabular}{|c|}
\hline Abbreviations Used \\
\hline 8-oxo-dG = 8-oxo-2'-deoxyguanosine \\
\hline $\mathrm{AA}=$ arachidonic acid \\
\hline $\begin{array}{c}\mathrm{ABL}=\text { Abelson murine leukemia viral } \\
\text { oncogene homologue }\end{array}$ \\
\hline $\mathrm{ALK}=$ anaplastic lymphoma kinase \\
\hline $\mathrm{ALS}=$ amyotrophic lateral sclerosis \\
\hline $\mathrm{AML}=$ acute myeloid leukemia \\
\hline $\begin{array}{c}\mathrm{AMPK}=5^{\prime} \text { adenosine monophosphate-activated } \\
\text { protein kinase }\end{array}$ \\
\hline $\mathrm{APC}=$ adenomatous polyposis coli \\
\hline $\mathrm{AQP} 3=$ aquaporin 3 \\
\hline $\mathrm{ATM}=$ ataxia-telangiectasia mutated \\
\hline AXIN $=$ axis inhibition protein 2 \\
\hline $\begin{array}{c}\mathrm{BCAR} 1=\text { breast cancer anti-estrogen resistance } \\
\text { protein } 1\end{array}$ \\
\hline $\mathrm{BIM}=\mathrm{Bcl}-2$ interacting mediator of cell death \\
\hline BRCA $1 / 2=$ breast can $^{1} / 21 / 2$ \\
\hline $\mathrm{Ca}^{2+}=$ calcium \\
\hline $\mathrm{CAMKII}=$ calcium/calmodulin-dependent kinase II \\
\hline $\mathrm{CK} 1=$ casein kinase \\
\hline $\mathrm{CLL}=$ chronic lymphocytic leukemia \\
\hline $\mathrm{CML}=$ chronic myelogenous leukemia \\
\hline $\mathrm{COX} 2=$ cyclooxygenase 2 \\
\hline $\mathrm{CREB}=\mathrm{cAMP}$ response element-binding protein \\
\hline $\mathrm{CXCR} 4=\mathrm{C}-\mathrm{X}-\mathrm{C}$ chemokine receptor type 4 \\
\hline cyt b588 = cytochrome b588 \\
\hline $\mathrm{DAG}=$ diacylglycerol \\
\hline $\mathrm{DDR}=\mathrm{DNA}$ damage response \\
\hline $\mathrm{ECM}=$ extracellular matrix \\
\hline $\mathrm{EGF}=$ epidermal growth factor \\
\hline $\mathrm{EMT}=$ epithelial/mesenchymal transition \\
\hline $\mathrm{FAD}=$ flavin adenine dinucleotide \\
\hline $\mathrm{FAK}=$ focal adhesion kinase \\
\hline $\mathrm{FAP}=$ familial adenomatous polyposis \\
\hline FLT3-ITD = FMS-like tyrosine kinase 3 \\
\hline with internal tandem duplications \\
\hline $\mathrm{FOX}=$ forkhead box \\
\hline $\mathrm{GAP}=$ small GTPase activator protein \\
\hline GDI $=$ guanine nucleotide disassociation inhibitor \\
\hline $\mathrm{GEF}=$ guanine nucleotide exchange factor \\
\hline $\mathrm{GPCR}=\mathrm{G}$ protein-coupled receptor \\
\hline GSK $3 \beta=$ glycogen synthase kinase $3 \beta$ \\
\hline $\mathrm{H}_{2} \mathrm{O}_{2}=$ hydrogen peroxide \\
\hline HER $2=$ human epidermal growth factor receptor 2 \\
\hline HIF- $1 \alpha=$ hypoxia inducible factor $1 \alpha$ \\
\hline $\mathrm{HNPCC}=$ hereditary nonpolyposis colorectal cancer \\
\hline $\mathrm{HSC}=$ hematopoietic stem cell \\
\hline
\end{tabular}




\section{Abbreviations Used (Cont.)}

$\mathrm{I} \kappa \mathrm{B} \alpha=$ nuclear factor of kappa light polypeptide gene enhancer in B cell inhibitor $\alpha$

ICAM $=$ intercellular adhesion molecule

IFN- $\gamma=$ interferon gamma

$\mathrm{IGF}=$ insulin growth factor

IKK $\alpha=$ inhibitor of nuclear factor kappa-B kinase subunit $\alpha$

$\mathrm{IL}=$ interleukin

IP3 = inositol triphosphate

iPSC $=$ induced pluripotent stem cell

IRAK = interleukin-1 receptor-associated kinase

$\mathrm{JNK}=\mathrm{c}$-Jun $\mathrm{N}$-terminal kinase

LIMK $=$ LIM kinase

LMW $=$ low-molecular-weight

LOX $=$ lipoxygenase

LT-HSC $=$ long-term hematopoietic stem cell

$\mathrm{MBL}=$ monoclonal $\mathrm{B}$ lymphocytosis

MCL1 = myeloid cell leukemia 1

$\mathrm{MMP}=$ matrix metalloproteinase

mRNA $=$ messenger RNA

$\mathrm{MS}=$ myeloplastic syndrome

MSCs $=$ mesenchymal stem/stromal cells

$\mathrm{NAC}=\mathrm{N}$-acetyl cysteine

$\mathrm{NF} \kappa \mathrm{B}=$ nuclear factor kappa-light-chain-enhancer of activated B cells

$\mathrm{NK}=$ natural killer

NOXA $1=$ NADPH oxidase activator 1 subunit

$\mathrm{NOXO} 1=\mathrm{NADPH}$ oxidase organizer 1 subunit

NSCLC $=$ nonsmall-cell lung cancer

$\mathrm{O}_{2}{ }^{\bullet-}=$ superoxide anion

$\mathrm{PAF}=$ platelet-activating factor

PDGF $=$ platelet-derived growth factor

$\mathrm{PD}-\mathrm{L}=$ programmed death ligand

PDPK1 = 3-phosphoinositide-dependent protein kinase-1

phox $=$ phagocytic oxidase

PI3K = phosphatidylinositol-4,5-bisphosphate 3-kinase

$P I 3 K \alpha=$ phosphatidylinositol-4,5-bisphosphate 3-kinase catalytic subunit $\alpha$

PIP2 = phosphatidylinositol (4,5)-biphosphate PIP3 = phosphatidylinositol (3,4,5)-triphosphate
$\mathrm{PKA}=$ protein kinase $\mathrm{A}$

$\mathrm{PKC}=$ protein kinase $\mathrm{C}$

$\mathrm{PLC}=$ phospholipase $\mathrm{C}$

PMA $=$ phorbol 12-myristate 13-acetate

$\mathrm{PTC}=$ papillary thyroid cancer

PtdIns $=$ phosphatidylinositol

PTEN $=$ phosphatase and tensin homologue

PTK $2 \beta=$ protein tyrosine kinase $2 \beta$

$\mathrm{PTP}=$ protein tyrosine phosphatase

PTPRJ/DEP-1 = protein tyrosine phosphatase, receptor type $\mathrm{J}$ redox $=$ reduction/oxidation

RGS4 $=$ regulator of $\mathrm{G}$ protein signaling 4

$\mathrm{RNAi}=\mathrm{RNA}$ interference

$\mathrm{ROCK}=\mathrm{RHO}$-associated, coiled-coil-containing protein kinase

ROS $=$ reactive oxygen species

$\mathrm{RTK}=$ tyrosine kinase receptor

SCLC $=$ small-cell lung cancer

$\mathrm{SD}=$ standard deviation

SDF-1 = stromal-derived factor 1

$\mathrm{SH}=\mathrm{SRC}$ homology

$\mathrm{SOD}=$ superoxide dismutase

$\mathrm{SP} 1=$ specificity protein 1

STAT5 $=$ signal transducer and activator of transcription 5

TGF $\beta=$ transforming growth factor $\beta$

TKS4 $=$ tyrosine kinase substrate with $4 \mathrm{SH} 3$ domains

TKS5 $=$ tyrosine kinase substrate with 5 SH3 domains

TLR4 $=$ Toll-like receptor 4

$\mathrm{TNF} \alpha=$ tumor necrosis factor alpha

TRAF6 $=$ TNF receptor-associated factor 6

TRAIL $=$ TNF-related apoptosis-inducing ligand

$\mathrm{TSH}=$ thyroid-stimulating hormone

TSHR $=$ thyroid-stimulating hormone receptor

$\mathrm{UTR}=$ untranslated region

$\mathrm{UV}=$ ultraviolet

VEGF $=$ vascular endothelial growth factor

$\mathrm{WNT}=$ wingless-type MMTV integration site family

WWTR1 $=$ WW domain containing transcription regulator 1

ZEB2 = zinc finger E-box binding homeobox 2 